Aus der Abteilung Psychiatrie und Psychotherapie

(Komm. Leiter: Prof. Dr. med. B. Bandelow)

im Zentrum Psychosoziale Medizin

der Medizinischen Fakultät der Universität Göttingen

\title{
Abhängigkeitsschweregrad, Komorbidität und Kognition im Benzodiazepinentzug
}

\author{
Inaugural-Dissertation zur Erlangung des Doktorgrades \\ der Medizinischen Fakultät \\ der Georg-August-Universität zu Göttingen
}

vorgelegt von

Anna Katrin Fischer

aus

Bremen

Göttingen 2013 
Dekan:

I. Berichterstatterin:

II. Berichterstatter/in:

III. Berichterstatter/in:

Tag der mündlichen Prüfung:

Prof. Dr. rer. nat. H. K. Kroemer

Prof. Dr. med. U. Havemann-Reinecke

Prof. Dr. rer. nat. H. K. Kroemer

Prof. Dr.med. U. Havemann-Reinecke





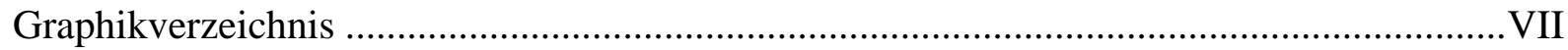

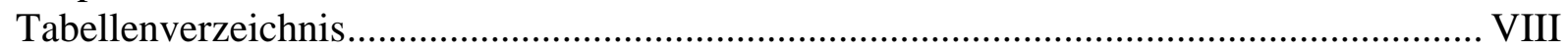

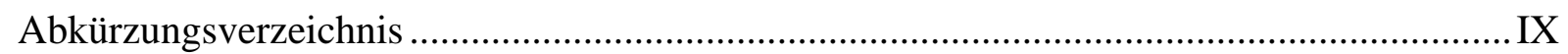

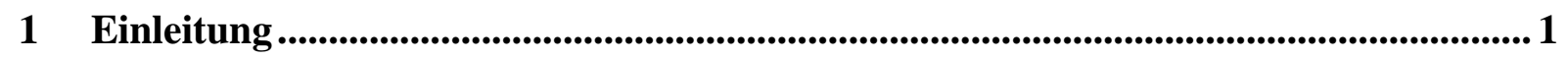

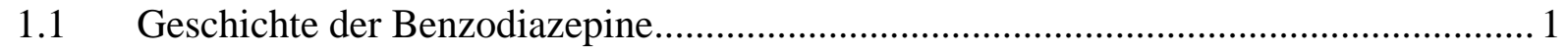

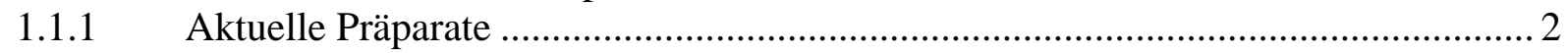

1.2 Wirkung und unerwünschte Wirkung der Benzodiazepine........................................... 2

1.2.1 Wirkung und klinische Anwendungsbereiche .................................................. 2

1.2.2 Unerwünschte Auswirkungen der Benzodiazepineinnahme .................................... 3

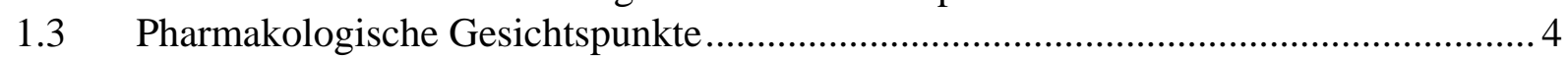

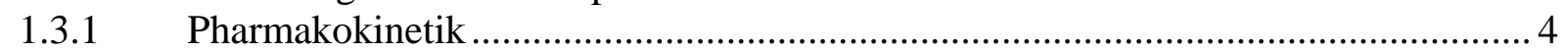

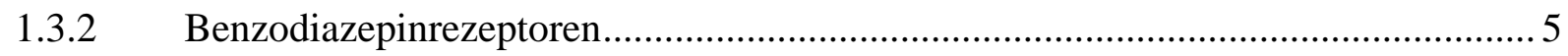

1.4 Benzodiazepinabusus und Benzodiazepinabhängigkeit ............................................ 6

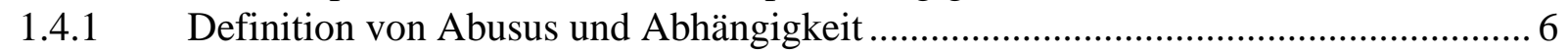

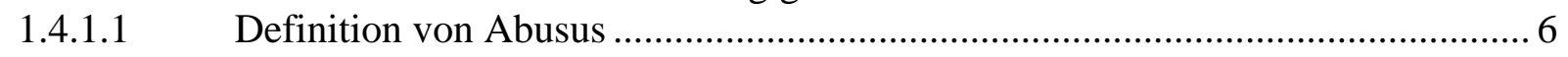

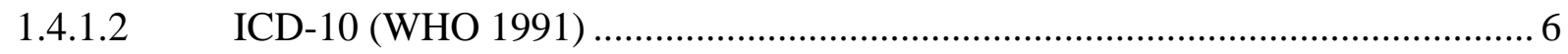

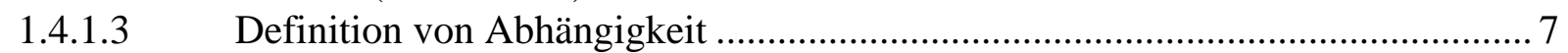

1.4.2 Benzodiazepinabusus und Benzodiazepinabhängigkeit ........................................ 8

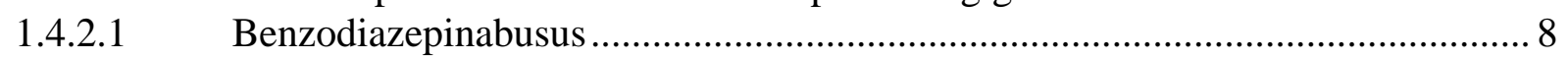

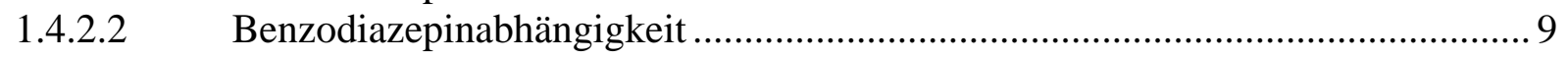

1.4.3 Komorbidität der Benzodiazepinabhängigkeit .................................................. 11

1.4.4 Epidemiologie/ Soziodemographie von Benzodiazepinabhängigkeit ..................... 11

1.5 Benzodiazepin- und komorbide Alkoholabhängigkeit ................................................ 12

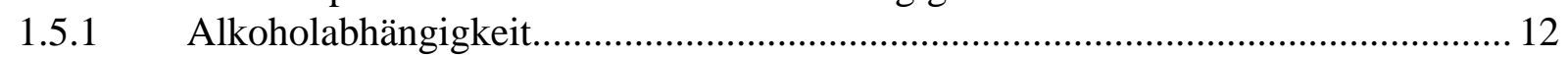

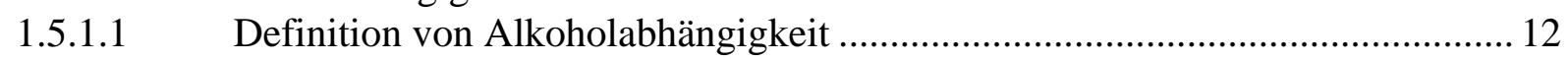

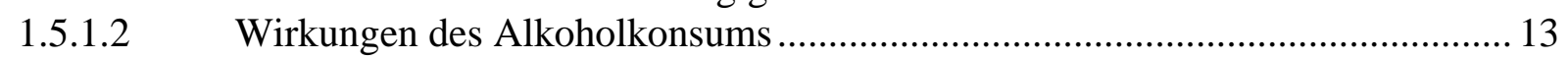

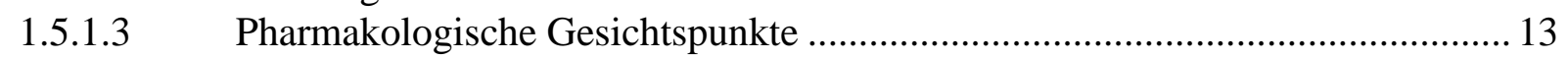

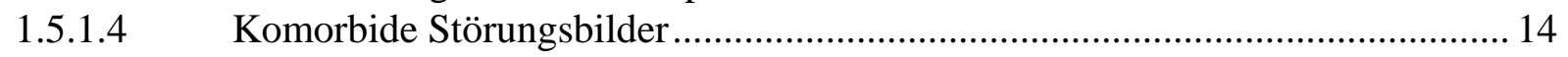

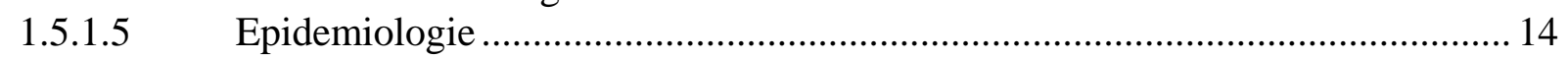

1.5.2 Pathogenese der Doppelabhängigkeit von Benzodiazepinen und Alkohol ............. 14

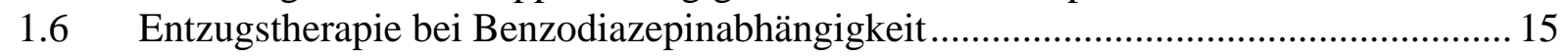

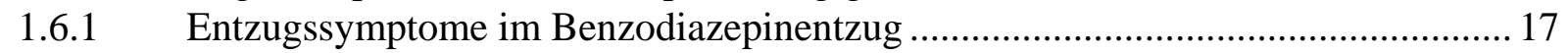

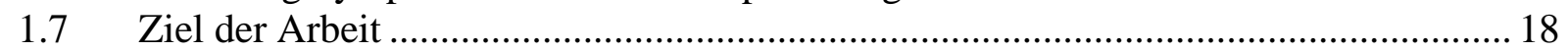

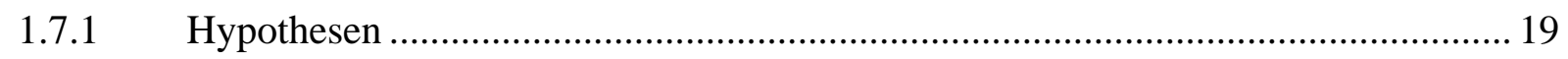

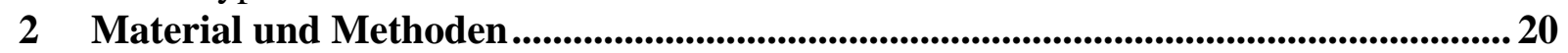

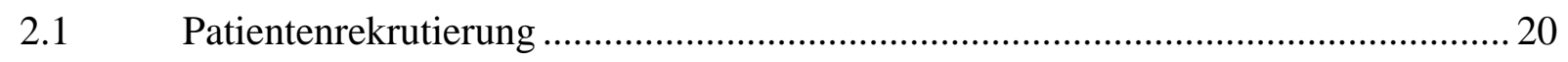

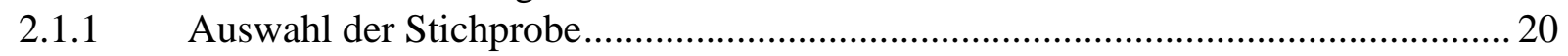

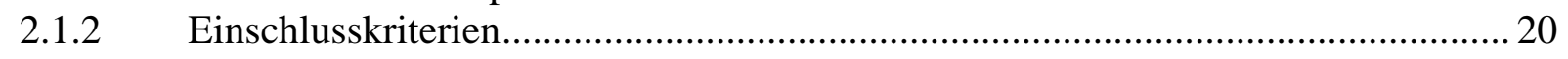

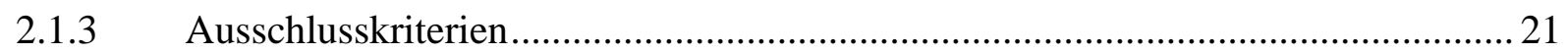

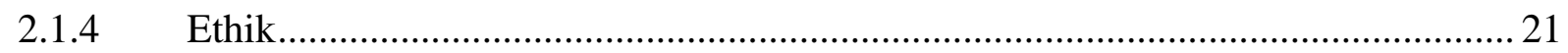

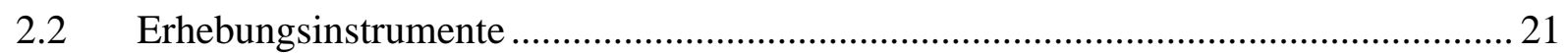

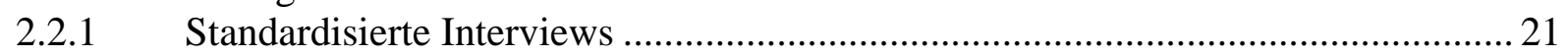

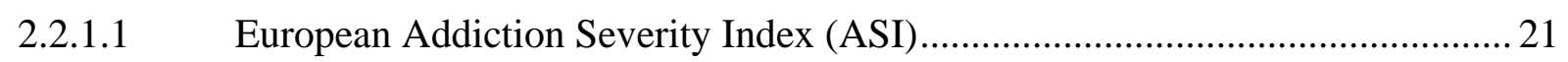

2.2.1.2 Diagnostisches Kurzinterview bei psychischen Störungen (MINI-DIPS) ......... 22

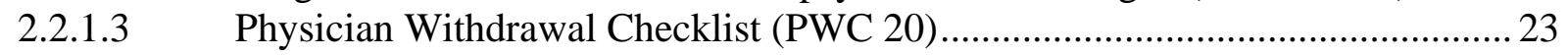

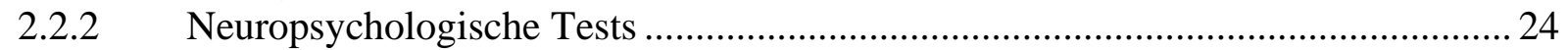

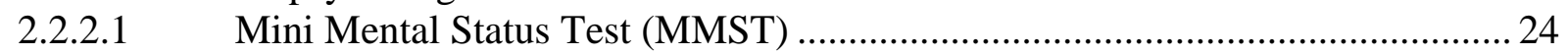

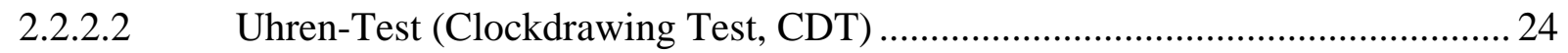

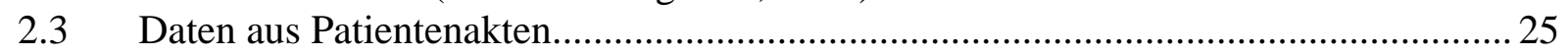

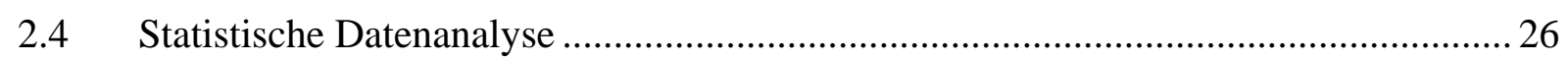




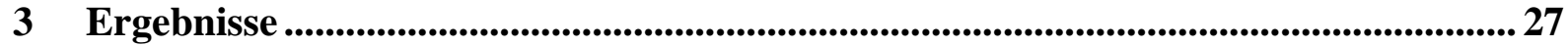

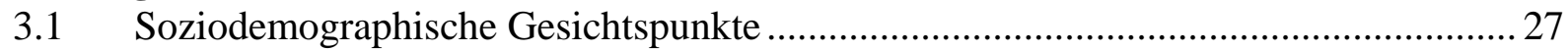

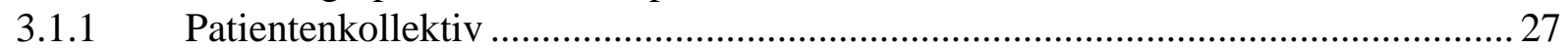

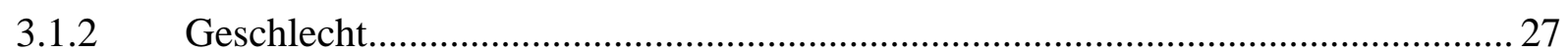

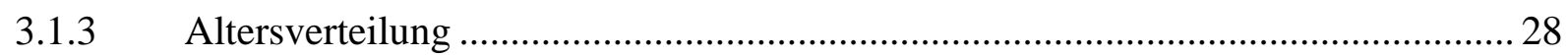

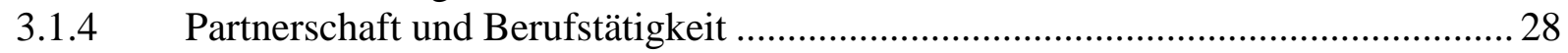

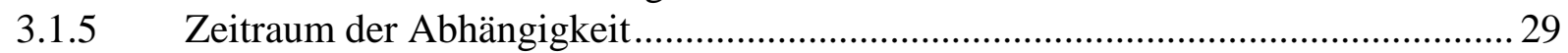

3.1.5.1 Zeitraum der Benzodiazepinabhängigkeit .................................................... 29

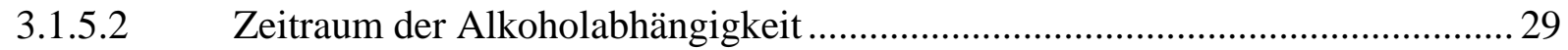

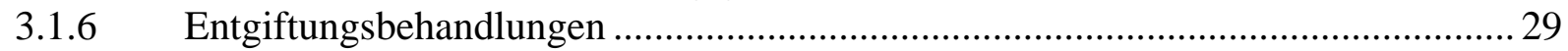

3.1.7 Benzodiazepindosis vor der Entzugstherapie .................................................. 29

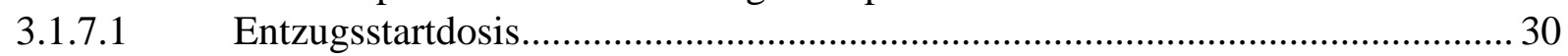

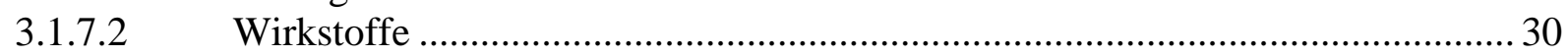

3.1.8 Zusammenfassung der Patientencharakteristika............................................... 31

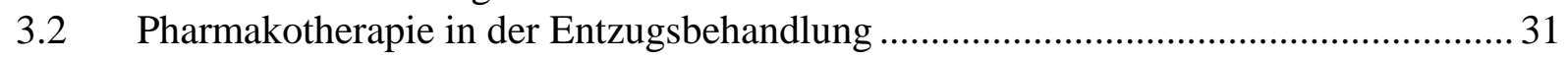

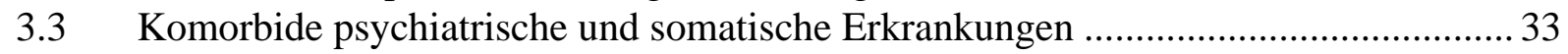

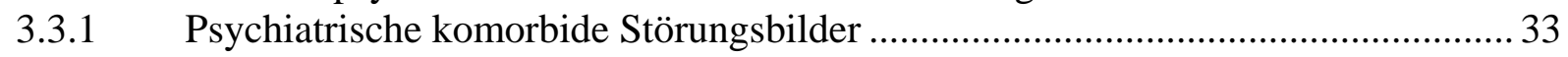

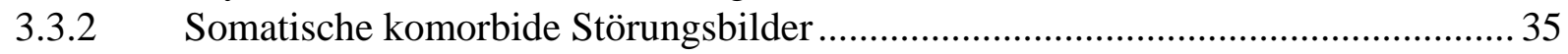

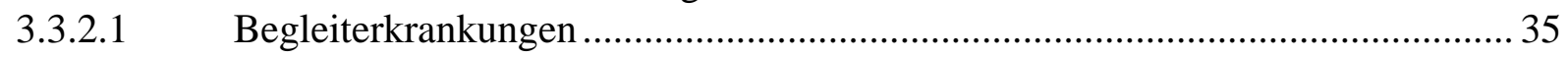

3.3.2.2 Leberwerte und abdomensonographische Befunde ........................................ 37

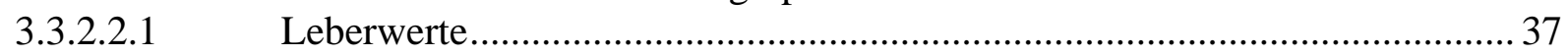

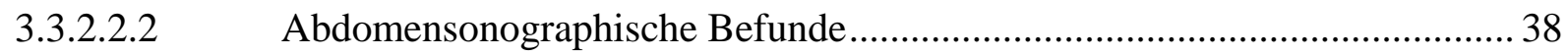

3.3.2.3 Bildgebende pathologische Befunde des Neurokraniums ................................ 39

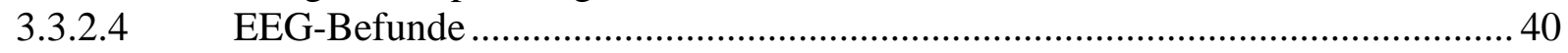

3.4 Kognitive Defizite benzodiazepinabhängiger Patienten ............................................. 40

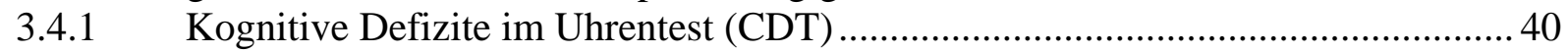

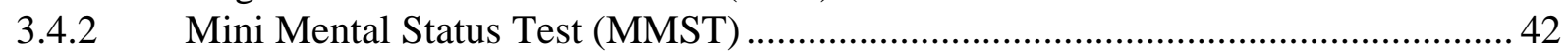

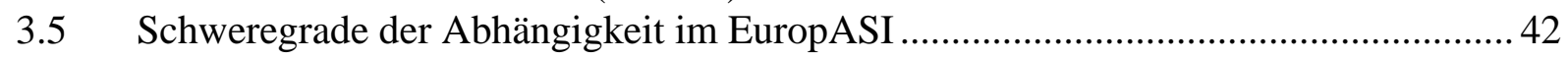

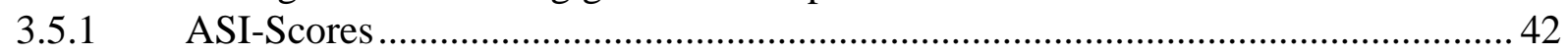

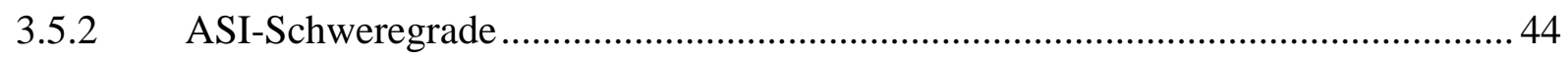

3.6 Entzugssymptome gemessen durch die Physician Withdrawal Checklist .................. 45

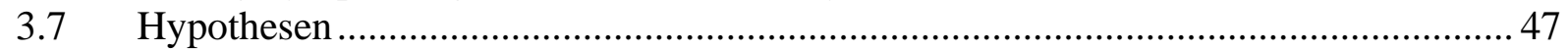

3.7.1 Hypothese 1: Benzodiazepinabhängige Patienten unterscheiden sich von benzodiazepin- und alkoholabhängigen Patienten im Benzodiazepinentzug hinsichtlich psychiatrischer und somatischer Komorbiditäten................................ 47

3.7.1.1 Vergleich komorbider psychiatrischer Störungsbilder ...................................... 47

3.7.1.2 Vergleich komorbider somatischer Störungsbilder ........................................... 48

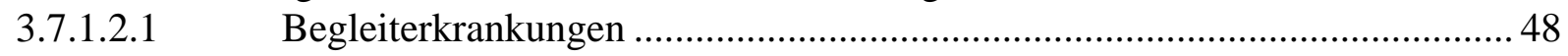

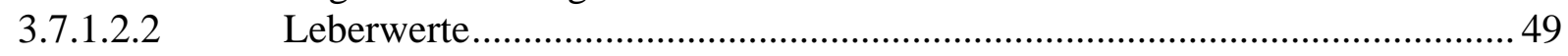

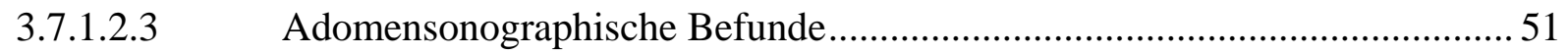

3.7.1.2.4 Bildgebende pathologische Befunde des Neurokraniums............................... 52

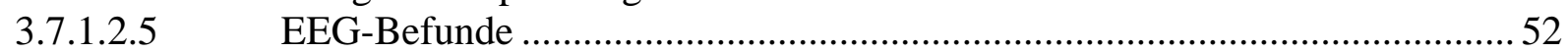

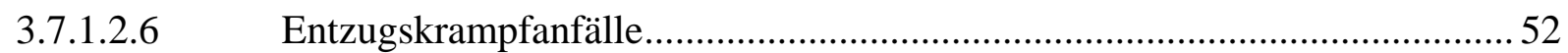

3.7.1.2.7 Zusammenfassung der Vergleiche zwischen den Gruppen............................. 52

3.7.2 Hypothese 2: Benzodiazepinabhängige Patienten haben bei Komorbidität mit einer Alkoholabhängigkeit im Benzodiazepinentzug ein höheres Ausmaß an kognitiven Defiziten als isoliert benzodiazepinabhängige Patienten ..................... 53

3.7.2.1 Vergleich kognitiver Defizite im Uhrentest (CDT) ......................................... 53

3.7.2.2 Vergleich kognitiver Defizite im MMST ....................................................... 53

3.7.2.2.1 Zusammenfassung der Vergleiche zwischen den Gruppen............................ 54 
3.7.3 Hypothese 3: Benzodiazepinabhängige Patienten unterscheiden sich von benzodiazepin- und alkoholabhängigen Patienten im Benzodiazepinentzug hinsichtlich des Schweregrades der Abhängigkeit . 55

3.7.3.1 Hypothese 3.1: Ein höherer Schweregrad der Abhängigkeit korreliert mit dem Vorhandensein von psychiatrischen und somatischen Komorbiditäten ............. 56

3.7.3.1.1 Korrelation Schweregrad der Abhängigkeit/ psychiatrische Komorbiditäten. .56

3.7.3.1.2 Korrelation des Schweregrades der Abhängigkeit mit somatischen Komorbiditäten...... .57

3.7.3.2 Hypothese 3.2: Ein höherer Schweregrad der Abhängigkeit korreliert mit einem höheren Ausmaß an kognitiven Defiziten im Benzodiazepinentzug ....... 58

3.7.3.3 Hypothese 3.3: Ein höherer Schweregrad der Abhängigkeit korreliert mit der Anzahl bisheriger Entgiftungsbehandlungen.

3.7.3.4 Hypothese 3.4: Das Vorhandensein psychiatrischer Komorbiditäten ..................... korreliert mit der Anzahl bisheriger Entgiftungsbehandlungen 60

3.7.3.5 Hypothese 3.5: Ein höherer ASI-Score des Problembereiches „körperlicher Zustand“ korreliert mit erhöhten Laborwerten, pathologischen Abdomensonographie-Befunden, cCT-, c-MRT-Befunden, EEG-Befunden oder anderen somatischen Begleiterkrankungen.

4 Diskussion

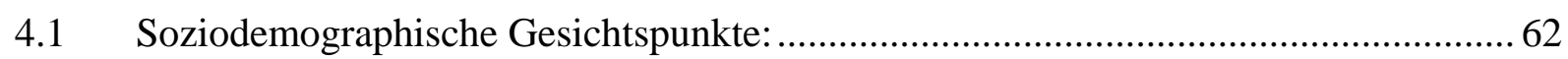

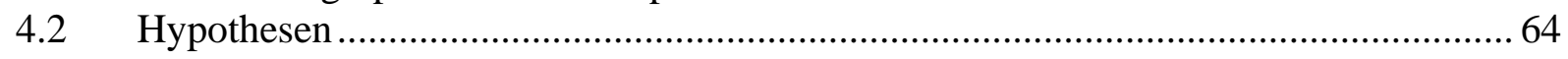

4.2.1 Hypothese 1: Benzodiazepinabhängige Patienten unterscheiden sich von benzodiazepin- und alkoholabhängigen Patienten im Benzodiazepinentzug hinsichtlich psychiatrischer und somatischer Komorbiditäten....

4.2.1.1 Komorbide psychiatrische Störungsbilder benzodiazepinabhängiger

Patienten. 65

4.2.1.2 Vergleich komorbider psychiatrischer Störungsbilder .....................................67

4.2.1.3 Komorbide somatische Störungsbilder benzodiazepinabhängiger Patienten ..... 68

4.2.1.3.1

4.2.1.3.2

4.2.1.3.3

4.2.1.4

4.2.1.4.1

4.2.1.4.2

4.2.1.4.3

4.2.1.4.4

4.2.1.5

4.2.2 Begleiterkrankungen 68

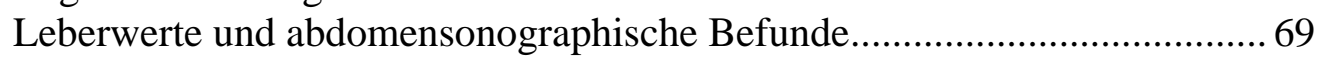

Bildgebende pathologische Befunde des Neurokraniums............................ 70

Vergleich komorbider somatischer Störungsbilder .......................................... 71

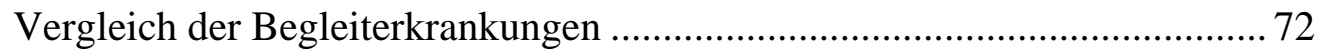

Vergleich der Leberwerte und der abdomensonographischen Befunde......... 72

Vergleich der bildgebenden pathologischen Befunde des Neurokraniums ... 73

Zusammenfassung der Vergleiche zwischen den Gruppen........................... 73

Limitierende Faktoren der Studienergebnisse ................................................ 73

Hypothese 2: Benzodiazepinabhängige Patienten haben bei Komorbidität mit einer Alkoholabhängigkeit im Benzodiazepinentzug ein höheres Ausmaß an kognitiven Defiziten als isoliert benzodiazepinabhängige Patienten ....................... 75

4.2.2.1 Kognitive Defizite benzodiazepinabhängier Patienten ...................................... 75

4.2.2.1.1 Kognitive Defizite im Uhrentest (CDT)................................................... 75

4.2.2.1.2 Kognitive Defizite im Mini Mental Status Test .............................................. 76

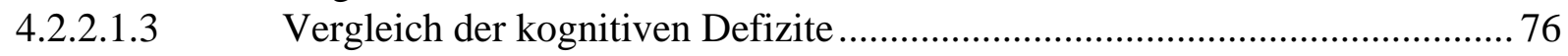

4.2.2.1.4 Beurteilung der kognitiven Defizite des gesamten Patientenkollektivs......... 76

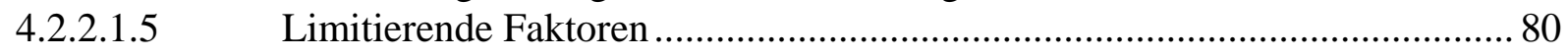

4.2.3 Hypothese 3: Benzodiazepinabhängige Patienten unterscheiden sich von benzodiazepin- und alkoholabhängigen Patienten im Benzodiazepinentzug hinsichtlich des Schweregrades der Abhängigkeit ............................................... 82

4.2.3.1 Schweregrad der Abhängigkeit des gesamten Patientenkollektivs..................... 82

4.2.3.2 Vergleich des Schweregrades der Abhängigkeit ............................................. 84 
4.2.4 Hypothese 3.1: Ein höherer Schweregrad der Abhängigkeit beider Patientengruppen korreliert mit dem Vorhandensein von psychiatrischen und somatischen Komorbiditäten

4.2.5 Hypothese 3.2: Ein höherer Schweregrad der Abhängigkeit korreliert mit einem höheren Ausmaß an kognitiven Defiziten im Benzodiazepinentzug...................... 85

4.2.6 Hypothese 3.3: Ein höherer Schweregrad der Abhängigkeit korreliert mit der Anzahl bisheriger Entgiftungsbehandlungen.

4.2.7 Hypothese 3.4: Das Vorhandensein psychiatrischer Komorbiditäten korreliert mit der Anzahl bisheriger Entgiftungsbehandlungen .

4.2.8 Hypothese 3.5: Ein höherer ASI-Score des Problembereiches „körperlicher Zustand“ korreliert mit erhöhten Laborwerten, pathologischen Abdomensonographie-Befunden, cCT-, c-MRT-Befunden, EEG-Befunden oder

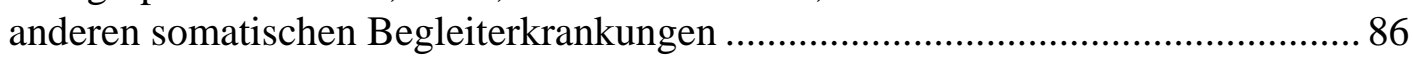

5 Zusammenfassung................................................................................................ 87

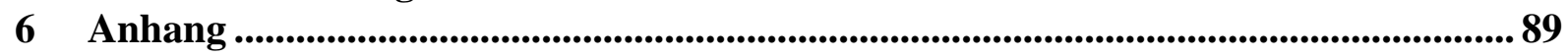

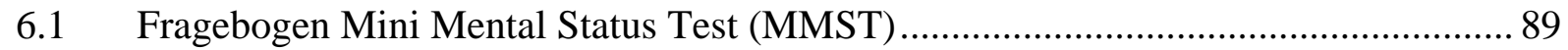

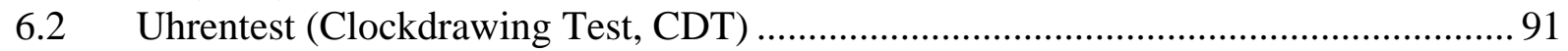

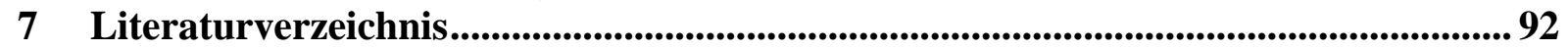

\section{Graphikverzeichnis}

Graphik 1: Verteilung der verschiedenen Substanzgruppen von Psychopharmaka in Gruppe A und Gruppe B zum Zeitpunkt der Befragung....

Graphik 2: Verteilung komorbider psychischer Störungen im gesamten Patientenkollektiv, in Gruppe A und Gruppe B

Graphik 3: Abbildung somatische Begleiterkrankungen im gesamten Kollektiv, in Gruppe A und Gruppe B

Graphik 4: Uhrentestergebnisse des gesamten Patientenkollektivs und der einzelnen Patientengruppen ....

Graphik 5: Vergleich der Ergebnisse des Uhrentest.

Graphik 6: Abbildung der ASI-Scores im gesamten Kollektiv, in Gruppe A und in Gruppe B

Graphik 7: Abbildung der ASI-Schweregrade im gesamten Kollektiv, in Gruppe A und in Gruppe B

Graphik 8: Vergleich der Mittelwerte der Rickels-Scores aufgelistet für jedes Symptom ..... 46

Graphik 9: Psychische komorbide Störungsbilder . 48

Graphik 10: Somatische komorbide Störungsbilder

Graphik 11: Leberwerte im Vergleich zwischen den Gruppen.....

Graphik 12: Harnsäurewerte im Vergleich zwischen den Gruppen. 50

Graphik 13: De-Ritis-Quotienten im Vergleich zwischen den Gruppen ............................... 51

Graphik 14: Abdomensonographische Befunde im Vergleich zwischen den Gruppen........... 51

Graphik 15: Ergebnisse des Uhrentest im Vergleich zwischen den Gruppen ..... .53

Graphik 16: Vergleich der Uhrentest-Scores angegeben in Mittelwerten von Patienten mit Somatisierungsstörungen und ohne Somatisierungsstörungen in Gruppe A...... 54

Graphik 17: ASI-Scores zum Schweregrad der Abhängigkeit .55 


\section{Tabellenverzeichnis}

Tabelle 1: Partnerschaft und berufliche Situation der gesamten Studiengruppe, in Gruppe A und Gruppe B

Tabelle 2: Vor der Entzugstherapie eingenommene Benzodiazepine

Tabelle 3: Zusammenfassung der Patientencharakteristika im gesamten Kollektiv, in Gruppe A und Gruppe B

Tabelle 4: Am häufigsten im Entzug verwendete Psychopharmaka in Gruppe A und Gruppe B

Tabelle 5: Komorbide psychische Erkrankungen des gesamten Kollektivs, in Gruppe A und in Gruppe B

Tabelle 6: Komorbide somatische Erkrankungen des gesamten Kollektivs, in Gruppe A und in Gruppe B

Tabelle 7: Leberwerte, De-Ritis-Quotient und Harnsäure des gesamten Kollektivs und in der Gruppe A und Gruppe B jeweils auch gesondert für die Teilnehmer, die Lorazepam einnahmen

Tabelle 8: Sono-Abdomen-Befunde des gesamten Kollektivs in Gruppe A und in Gruppe B

Tabelle 9: Pathologische Befunde des Neurokraniums des gesamten Kollektivs, in Gruppe A und in Gruppe B

Tabelle 10: Korrelationen im Vergleich hirnathrophischer Veränderungen mit den Ergebnissen des MMST und Uhrentest im gesamten Patientenkollektiv

Tabelle 11: Abbildung der ASI-Scores der verschiedenen Problembereiche im gesamten Kollektiv, in Gruppe A und in Gruppe B

Tabelle 12: Abbildung der ASI-Schweregrade der verschiedenen Problembereiche im gesamten Kollektiv, in Gruppe A und in Gruppe B.

Tabelle 13: Abbildung der Rickels-Scores

Tabelle 14: Signifikante Korrelationen im Vergleich der Parameter der Schwere der Abhängigkeit mit somatischen Komorbiditäten

Tabelle 15: Korrelationen im Vergleich der Parameter der Schwere der Abhängigkeit mit den Ergebnissen des Mini Mental Status Test und der Uhrentests.

Tabelle 16: Vergleich der Ergebnisse von MMST und Uhrentest von Patienten mit komorbiden psychiatrischen Erkrankungen und ohne komorbide psychiatrische Erkrankungen

Tabelle 17: Korrelationen mit Wahrscheinlichkeit im Vergleich der Parameter der Schwere der Abhängigkeit mit der Anzahl stattgefundener Entgiftungsbehandlungen.... 60

Tabelle 18: Korrelationen im Vergleich des Vorhandenseins von psychiatrischen Komorbiditäten mit der Anzahl stattgefundener Entgiftungsbehandlungen 


\section{Abkürzungsverzeichnis}

APA: American Psychological Association

CCT: $\quad$ Kraniale Computertomographie

CDT: Clockdrawing Test

CMRT: Kraniale Magnetresonanztomographie

DHS: Deutsche Hauptstelle für Suchtfragen

DSM: Diagnostic and Statistical Manual of Mental Disorders

EEG: $\quad$ Elektroenzephalographie

EuropASI: $\quad$ European Addiction Severity Index

GABA: $\quad$ Gamma-Aminobuttersäure

Gamma-GT: Gamma-Glutamyl-Transferase

GOT: Glutamat-Oxalazetat-Transaminase

GPT: $\quad$ Glutamat-Pyruvat-Transaminase

ICD: International Classification of Diseases and Related Health Problems

IMS: $\quad$ Institut für Medizinische Statistik

MINI-DIPS: Diagnostisches Kurzinterview bei psychischen Störungen

MMST: $\quad$ Mini Mental Status Test

n: Größe der Stichprobe

NESARC: $\quad$ National Epidemiologic Survey on Alcohol and Related Conditions

p: $\quad$ Statistische Wahrscheinlichkeit

PTBS: Posttraumatische Belastungsstörung

PWC: $\quad$ Physician Withdrawal Checklist

r: Regressionskoeffizient

SD: $\quad$ Standard Deviation

SSRI: Selective Serotonin Reuptake Inhibitor

U: U-Wert des Mann-Whitney-U-Tests

UMG: Universitätsmedizin Göttingen

WHO: Weltgesundheitsorganisation 


\section{Einleitung}

\subsection{Geschichte der Benzodiazepine}

Die Synthese der ersten Benzodiazepine gelang Ende der 50er Jahre der Firma Hoffmann-La Roche. Das erste Präparat, das klinisch genutzt werden konnte, war das Chlordiazepoxid, das im Jahr 1960 auf den Markt kam. 1963 folgte das Diazepam, das mittlerweile zur Standardsubstanz wurde (LADER 1991). Vor den Benzodiazepinen waren die Barbiturate die meist verwendeten synthetischen Sedativa. Nachteile dieser Substanzen waren jedoch ein hohes Abhängigkeitspotential und eine geringe therapeutische Breite, verbunden mit der Gefahr der toxischen Überdosierung. Der direkte Vorgänger der Benzodiazepine war das Meprobamat, es wurde 1950 entwickelt und weltweit als Anxiolytikum verwendet, aber auch diese Substanz zeigte ein sehr hohes Abhängigkeitspotential.

Der Gebrauch der Benzodiazepine stieg in den 60er und den frühen 70er Jahren dramatisch an. Aufgrund ihrer guten Wirksamkeit als Tranquilizer und Hypnotika bei schnellem Wirkungseintritt, geringen Nebenwirkungen, geringer Toxizität und großer therapeutischer Breite kam es zu einer raschen Verbreitung und hohen Akzeptanz in der Ärzteschaft (LADER 1991, ROSENBAUM 2005). Binnen kürzester Zeit wurden sie zu den am häufigsten verschriebenen Medikamenten der Gruppe der Sedativa/ Hypnotika, obwohl bereits ein Jahr nach Markteinführung bekannt wurde, dass hohe Dosen eine barbituratähnliche Abhängigkeit erzeugen können (HOLLISTER et al. 1961). In den Jahren von 1969 bis 1981 war Diazepam das am häufigsten verschriebene Medikament in Amerika. Allein im Jahr 1978 wurden ca 2,3 Milliarden Tabletten verkauft (LADER 2011). Während der 80er Jahre gab die öffentliche Meinung über das Potential zum Missbrauch und die körperliche Abhängigkeit bei Langzeiteinnahme Grund zu zahlreichen kontroversen Diskussionen. Als Reaktion auf das zunehmend in Erscheinung tretende Abhängigkeitspotential der Benzodiazepine, erließen einige Staaten (zum Beispiel New York) 1989 eine Legislation (triplicate prescription program), um die willkürliche Verschreibung von Benzodiazepinen zu minimieren. Folge dieser Legislation war ein Rückgang in der Verschreibung von Benzodiazepinen und eine starke Zunahme der Verschreibung älterer Substanzen mit niedrigerer therapeutischer Sicherheit (ROSENBAUM 2005) und einer signifikanten Zunahme von sedativ hypnotischen Überdosen dieser Substanzen (HOFFMAN et al. 1991).

Benzodiazepine sind bis zum heutigen Tag unverzichtbare Arzneimttel in der Gesamtmedizin. Dennoch sollte ihre Verschreibung nur nach eindeutiger Indikationsstellung und mit kurzer Dauer erfolgen. Alle Arzneimittel vom Benzodiazepintyp sind rezeptpflichtig, dem Arzt 
kommt daher eine wichtige Kontrollfunktion zu. 1985 zeigte eine Studie in den USA, dass Benzodiazepinrezepte hauptsächlich von Hausärzten ausgestellt werden. $59 \%$ aller Tranquilizer wurden von Hausärzten verschrieben, Psychiater verabreichten nur 19\% dieser Medikamente (GONZALES et al. 1992). In den letzten Jahren sind die Verordnungen von Benzodiazepinen im Rahmen der gesetzlichen Krankenversicherung zurückgegangen, der Anteil der privat verordneten Mittel steigt jedoch weiter an. Die im Jahre 2008 verkauften Mengen reichen immer noch aus, um die aktuell ca 1,1-1,2 Millionen Abhängigen in Deutschland zu versorgen. So waren das 2008 ca. 19,9 Millionen Packungen von Schlaf- und Beruhigungsmitteln mit Benzodiazepin- oder benzodiazepinähnlichen Wirkstoffen. Zunehmende Bedeutung erlangen hierbei auch die sogenannten Z-Drugs mit den Wirkstoffen Zolpidem, Zoplicon oder auch Zalepon (GLAESKE 2010).

\subsubsection{Aktuelle Präparate}

Die im Jahr 2008 in Deutschland am häufigsten verkauften Präparate aus der Gruppe der benzodiazepinhaltigen Hypnotika/ Sedativa waren:

- Lendormin mit dem Wirkstoff Brotizolam,

- Noctamid mit dem Wirkstoff Lormetazepam

- Radedorm mit dem Wirkstoff Nitrazepam,

- Planum mit dem Wirkstoff Temazepam

- Rohypnol mit dem Wirkstoff Flunitrazepam.

Noch vor diesen Substanzen rangierten Präparate, die Zopiclon oder Zolpidem enthielten. Die laut Institut für Medizinische Statistik (IMS) am häufigsten verkauften Tranquilizer waren:

- Diazepam-ratiopharm mit dem Wirkstoff Diazepam

- Tavor mit dem Wirkstoff Lormetazepam

- Bromazanil-Hexal mit dem Wirkstoff Bromazepam

- Oxazepam-ratiopharm mit dem Wirkstoff Oxazepam

- Adumbran mit dem Wirkstoff Oxazepam (IMS 2009).

\subsection{Wirkung und unerwünschte Wirkung der Benzodiazepine}

\subsubsection{Wirkung und klinische Anwendungsbereiche}

Benzodiazepine gehören zur Gruppe der Sedativa/ Hypnotika sowie der Tranquillanzien. Sie alle setzen die Wahrnehmungsfähigkeit, die Spontaneität und körperliche Aktivität herab. Benzodiazepine sind die am meisten verschriebenen Substanzen dieser Gruppe und haben 
folgende therapeutische Wirkungen: Anxiolyse, hypnotische Wirkung, sedative Wirkung, Muskelrelaxation, antikonvulsive und amnestische Wirkung (POSER W et al. 2006). Anwendung finden Benzodiazepine in erster Linie in der hausärztlichen Praxis oder Allgemeinmedizin sowie in der Psychiatrie und Neurologie.

Ihr rascher Wirkungseintritt bei geringer Toxizität, verbunden mit den genannten therapeutischen Eigenschaften, machen sie zu Medikamenten, die in der Akutbehandlung von Patienten mit Angsterkrankungen, insbesondere bei psychotischen Ängsten und bei schweren Depressionen eine wichtige Rolle spielen (ROSENBAUM 2005). Ihr ursprünglicher Einsatz lag in der Behandlung von Angst- und Schlafstörungen. Weitere wichtige Indikationsgebiete sind beispielsweise die Anästhesie, die amnestische Effekte in der Prämedikation nutzt, oder die Innere Medizin wo Benzodiazepine z.B. zur Sedierung vor endoskopischen Untersuchungen verwendet werden. Angewendet werden Benzodiazepine mittlerweile in nahezu allen medizinischen Fachrichtungen gegen Unruhe und Angstzustände.

\subsubsection{Unerwünschte Auswirkungen der Benzodiazepineinnahme}

Generell zeigen sich unerwünschte Effekte der Benzodiazepineinnahme entgegengesetzt dem gewünschten klinischen Wirkungsprofil, das Anxiolyse, hypnotisch-sedierende Effekte und amnestische Effekte beinhaltet.

Unerwünscht aber können nach akuter Anwendung Sedation, anterograde Amnesien, psychomotorische Verlangsamung und Schwierigkeiten bei der Erfassung neuer Informationen sein (BUFFET-JEROTT 1998). Frühe Beschreibungen bezeichneten diese Effekte auf das Gedächtnis auch als traveler's amnesia, da Patienten unter der Einnahme von Benzodiazepinen problemlos in der Lage waren, bekannte Aufgaben zu bewältigen, es ihnen jedoch nicht möglich war, spezifische Details, die die Speicherung neuer Informationen erfordert hätten, wiederzugeben (BARBEE 1993). Bei langwirksamen Benzodiazepinen besteht die Gefahr von sogenannten hangover-Effekten. Hierbei führt der langsame Abbau der Substanzen zu einem „Überhang“, der sich am nächsten Morgen in Form von Müdigkeit und Abgeschlagenheit bemerkbar macht. Insbesondere bei älteren Menschen führt dies zu einer erhöhten Gefahr von häuslichen Unfällen, wie z.B. Stürzen mit Hüftfrakturen (RAY et al. 1987). Teilweise scheinen diese Effekte durch die zu Beginn der Behandlung noch stark sedierende Wirkung der Benzodiazepine bedingt und im Rahmen von Toleranzentwicklung nachzulassen; sie können aber durch die häufig langen Halbwertszeiten der Substanzen und ihrer wirksamen Metaboliten zu Akkumulation führen und persistieren.

Kontrovers werden mögliche persistierende kognitive Defizite diskutiert. Kognitive Funktionen, über deren Beeinträchtigung auch unter Langzeit-Einnahme von Benzodiazepinen 
berichtet wird, sind das visuell- räumliche Vorstellungsvermögen, visuomotorische Fähigkeiten (GOLOMBOK et al. 1988, TATA et al. 1994), die motorische Koordination, die psychomotorische Geschwindigkeit, die Geschwindigkeit der Informationsverarbeitung, verbales Lernen, Konzentration und Reaktionszeit (GOLOMBOK et al. 1988, BUFFETJEROTT et al. 1998, BARKER et al. 2004a). Diese Wirkungen auf kognitive Funktionen können zu massiven Problemen im Alltag der Betroffenen führen. So wird im Fall älterer Patienten beispielsweise über eine Zunahme der Wahrscheinlichkeit von Verkehrsunfällen, ausgelöst durch die weiter bestehende Einschränkung psychomotorischer Fähigkeiten, berichtet (HEMMELGARN et al. 1997). Besonders bei älteren Menschen kann eine Akkumulation der Plasmaspiegel Symptome einer Demenz vortäuschen und fälschlicherweise als Alzheimerdemenz ausgelegt werden (O’BRIEN 2005). Studien zeigten, dass sich benzodiazepinabhängige Patienten ihrer kognitiven Defizite oftmals kaum bewusst waren oder diese stark unterschätzten (BARBEE 1993, BUFFET-JEROTT und STEWART 2002).

Andere Studien, die sich ebenfalls mit einer möglichen Beeinflussung kognitiver Fähigkeiten unter langzeitiger Benzodiazepineinnahme beschäftigten, dabei allerdings nur kleine Patientengruppen untersuchten, sahen keinen Hinweis auf dauerhafte kognitive Defizite (LUCKI et al. 1986). Eine andere unerwünschte Auswirkung langzeitiger Einnahme von Benzodiazepinen ist die Entwicklung von Abhängigkeit mit entsprechenden Entzugssymptomen (POSER W et al. 2006).

\subsection{Pharmakologische Gesichtspunkte}

\subsubsection{Pharmakokinetik}

Die einzelnen Benzodiazepine unterscheiden sich vor allem bezüglich ihrer Pharmakokinetik, weniger ihrer Pharmakodynamik. So lassen sich beispielsweise lang-, mittel- und kurzwirksame Benzodiazepine abgrenzen (POSER W und POSER S 1996):

- Langwirksame Benzodiazepine: Diazepam, Flurazepam, Lorazepam, Nitrazepam, Flunitrazepam

- Kurz bis mittellang wirksame Benzodiazepine: Oxazepam, Temazepam, Lormetazepam

- Sehr kurz wirksame Benzodiazepine: Triazolam, Midazolam, Brotizolam.

Diese Abgrenzung ist insbesondere in Hinblick auf die Therapiesicherheit von Bedeutung. So zeigen, wie bereits oben beschrieben, langwirksame Benzodiazepine die Tendenz zu hangover-Effekten (POSER W und POSER S 1996). Auch für das Potential der Entwicklung einer Abhängigkeit von Benzodiazepinen spielt die Pharmakokinetik unter vielen anderen Faktoren eine Rolle. So weisen beispielsweise Präparate mit einer raschen Anflutung der 
Substanz und ihrer Metaboliten ein hohes Suchtpotential auf (O’BRIEN 2005). Viele Benzodiazepine sind sogenannte „Prodrugs“, wobei erst der vom Körper gebildete Metabolit wirksam ist, was das Verständnis der Kinetik erschwert. Insgesamt haben Benzodiazepine eine große therapeutische Breite. Ihre akute Toxizität ist dabei gering. Vergiftungen treten selbst bei Einnahme massiver Überdosen kaum auf (POSER W und POSER S 1996).

\subsubsection{Benzodiazepinrezeptoren}

Benzodiazepine entfalten ihre Wirkungen über einen spezifischen Rezeptor im zentralen Nervensystem, den GABA (gamma-Aminobuttersäure)-A-Rezeptor. GABA ist der wichtigste inhibitorische Neurotransmitter im Zentralen Nervensystem, bis zu 30\% aller Synapsen des Gehirns sind GABAerg. Er steht in einem dynamischen Gleichgewicht mit dem exzitatorischen Transmitter Glutamat (ROY-BYRNE 2005).

Der GABA-A-Rezeptor ist ein aus fünf Untereinheiten aufgebautes Pentamer, das einen zentralen Chlorid-Ionen-Kanal umschließt. Der Rezeptor besteht dabei aus zwei alpha-, zwei beta- und einer gamma-Untereinheit. Verschiedenste Isoformen dieser Untereinheiten konnten bisher identifiziert werden. So existieren mindestens sechs Isoformen der alphaUntereinheit (a1-a6) und drei Isoformen der beta- und gamma-Untereinheit (OLSEN und SIEGHARDT 2008). Die Bindungsstelle für GABA liegt jeweils zwischen der alpha- und der beta-Untereinheit. Die Bindung von GABA an den Rezeptor führt zu einer Öffnung des Chlorid-Ionen-Kanals mit folgender Hyperpolarisation des Zellinneren und damit zu einer Steigerung der neuronalen Inhibition. Die Bindungsstelle der Benzodiazepine liegt zwischen der alpha- und der gamma-Untereinheit. Die Bindung der Benzodiazepine an den Rezeptor führt zu einer Frequenzerhöhung der Kanalöffnung in Gegenwart von GABA und vestärkt somit die inhibitorische GABA-Wirkung (SAARI et al. 2011). Die verschiedenen Subtypen der GABA-A-Rezeptoren unterscheiden sich in Ihrer Affinität zu GABA sowie auch in ihrer Affinität zu Benzodiazepinen. Ebenso zeigten sich Zusammenhänge zwischen der Struktur des GABA-A-Rezeptors und den verschiedenen Wirkungen der Benzodiazepine (ROYBYRNE 2005). So scheint der GABA-A-Rezeptor mit den Untereinheiten 2 alpha 1-2, beta 2-1, gamma 2, insbesondere die sedative Wirkung der Benzodiazepine zu vermitteln (RUDOLPH et al. 1999, LYDIARD 2003), während Rezeptoren mit der alpha-2-Untereinheit eher anxiolytische Wirkungen vermitteln (LOW et al. 2000).

Die neuronale Basis der Entstehung der Abhängigkeit von Benzodiazepinen bleibt schwer fassbar. Die längerfristige Anwendung von Benzodiazepinen scheint, bedingt durch die dauerhafte Disinhibition GABAerger Neurone, zu einer erhöhten Sensitivität exzitatorischer glutamerger Neurone zu führen. Diese verstärkt Glutamat-gesteuerte Neurotransmission kann 
zu Toleranz gegenüber den Benzodiazepinen und zu Entzugssymptomen, wie verstärkter Angst oder auch anderen Symptomen, bei Beendigung der Einnahme führen (ALLISON und PRATT 2003). Die süchtig machende Komponente der Benzodiazepine scheint genau wie im Fall der Opioide oder Cannabinoide aber insbesondere durch Ihre Wirkung auf das dopaminerge Belohnungssystem bedingt. So führen diese Suchtstoffe über ihre disinhibitorische Wirkung auf mehrere hintereinandergeschaltete GABAerge Neurone zu einer Disinhibition dopaminerger Neurone. Diese Neurone liegen in der ventralen tegmentalen Zone des Zentralen Nervensystems und interagieren mit Neuronen des limbischen Systems und des kortikalen Systems. Insbesondere der Nucleus accumbens, als Verbindung zwischen limbischem und motorischem System, scheint hierbei wichtig für das Motivationsverhalten (HAVEMANN-REINECKE 1998, WOLF 2003).

Diese disinhibitorische Wirkung wird verstärkt über Rezeptoren mit der Untereinheit alpha 1 vermittelt. Studien mit transgenen Mäusen zeigten, dass bei Fehlen der alpha-1-Untereinheit keine erhöhte Dopaminausschüttung mehr nachgewiesen werden kann. Dementsprechend fehlt hier die Abhängigkeit auslösende Komponente. Insgesamt führen Stoffe, wie die Benzodiazepine, zu Anpassungsvorgängen im Bereich der Synapsen des mesolimbischen Systems (TAN et al. 2010).

Die Effekte der Benzodiazepine können durch Antagonisten, wie z.B. Flumazenil, aufgehoben werden, die keine oder nur sehr geringe pharmakologische Aktivität aufweisen (ROY-BYRNE 2005).

\subsection{Benzodiazepinabusus und Benzodiazepinabhängigkeit}

\subsubsection{Definition von Abusus und Abhängigkeit}

Neben den Formen des normalen und therapeutischen Konsums bestimmter Substanzgruppen kann auf Basis der Klassifikation der American Psychological Association (APA) und der WHO (Weltgesundheitsorganisation) zwischen schädlichem Gebrauch bzw. Abusus und Abhängigkeitssyndrom unterschieden werden.

\subsubsection{Definition von Abusus}

\subsubsection{ICD-10 (WHO 1991)}

Abusus oder Missbrauch wird nach dem DSM-IV (APA 1994) als ein maladaptives Muster des Gebrauchs der entsprechenden Substanz definiert. Trotz des Wissens um anhaltende oder wiederkehrende soziale, berufliche, psychische oder körperliche Probleme, die durch die 
Substanzeinnahme ausgelöst werden, wird die Einnahme fortgesetzt. Ein weiteres Kriterium ist der wiederholte Gebrauch in Situationen, in denen es zu einer körperlichen Gefährdung durch die Substanzeinnahme kommen kann.

Auch nach den Leitlinien ICD 10 (WHO 1991), bezeichnet der Abusus oder schädliche Gebrauch ein Konsummuster mit schädlichen Gesundheitsfolgen für Psyche oder Physis des Konsumenten. Dazu zählen sowohl körperliche als auch psychische Störungen als Folge des Konsums. Wiederholter Abusus kann dabei zu Abhängigkeit führen.

\subsubsection{Definition von Abhängigkeit}

Nach dem DSM-IV (APA 1994) kann die Diagnose "Abhängigkeit“ gestellt werden, wenn während der letzten zwölf Monate mindestens drei der folgenden Kriterien gleichzeitig erfüllt waren:

1. Nachweis einer Toleranz. Mit dem Verlangen nach Dosissteigerung und verminderter Wirkung bei gleichbleibender Dosis.

2. Entzugssymptome mit Wiederaufnahme des Substanzkonsums zur Linderung oder Vermeidung der Symptome.

3. Konsum häufig in größeren Mengen als beabsichtigt.

4. Andauernder Wunsch oder mindestens ein erfolgloser Versuch zur Dosisverminderung oder Abstinenz.

5. Hoher Zeitaufwand für die Beschaffung, den Konsum oder die Erholung von der Substanz.

6. Einschränkung sozialer, beruflicher oder Freizeitaktivitäten aufgrund des Substanzmissbrauchs.

7. Fortsetzung des Konsums, trotz Einsicht in körperliche oder psychische Probleme.

Auch nach den Leitlinien ICD 10 (WHO 1991) ist das Abhängigkeitssyndrom klar definiert. Die Diagnose Abhängigkeit kann gestellt werden, wenn während der letzten zwölf Monate drei oder mehr der sechs folgenden Kriterien gleichzeitig erfüllt waren:

1. Ein starker Wunsch oder eine Art Zwang, eine psychotrophe Substanz zu konsumieren.

2. Verminderte Kontrollfähigkeit bezüglich des Beginns, der Beendigung und der Menge des Konsums.

3. Ein körperliches Entzugssyndrom bei Beendigung oder Reduktion des Konsums, mit substanzspezifischen Entzugssymptomen oder Ersatzkonsum, um Entzugssymptome zu mildern oder zu vermeiden

4. Wirkungsverlust und Toleranzentwicklung, so dass höhere Mengen erforderlich sind, um die ursprüngliche Wirkung hervorzurufen.

5. Vernachlässigung anderer Interessen zugunsten des Substanzkonsums, wegen erhöhtem Zeitaufwand, um die Substanz zu beschaffen, zu konsumieren oder sich von den Folgen zu erholen.

6. Anhaltender Substanzkonsum,trotz Nachweis eindeutiger schädlicher Folgen. 
Entsprechend den Leitlinien ICD 10 (WHO 1991) kann das Abhängigkeitssyndrom unterschieden werden in vorwiegend psychische oder vorwiegend körperliche Abhängigkeitssymptome. Psychische Abhängigkeit bezeichnet das Verlangen, den bekannten Effekt eines Suchtstoffs erneut zu erfahren und den Konsum dieses Stoffes fortzusetzen. Im englischen hat sich hierfür der Begriff des craving als Ausdruck von Verlangen nach dem Stoff geprägt. Ausdruck von psychischer Abhängigkeit ist ein auf die Beschaffung des Suchtstoffes ausgerichteter Lebensstil, hinter dem alle anderen Werte zurückzustehen haben (POSER W und POSER S 1996). Von körperlicher Abhängigkeit kann gesprochen werden, wenn durch das Absetzen einer Medikation eine Entzugssymptomatik auftritt, die einen vorhersehbaren Beginn und Verlauf hat und sich diese durch die Wiedereinnahme des Medikamentes beenden lässt. Als weiteres Symptom körperlicher Abhängigkeit gilt die Toleranzentwicklung, was bedeutet, dass einzelne bis alle Wirkqualitäten eines Stoffes im Verlauf abnehmen (ROSENBAUM 2005). Im Gegensatz zu früher müssen die klassischen Symptome der körperlichen Abhängigkeit, nämlich Toleranz und Entzugserscheinungen zur Diagnosefindung nicht mehr unbedingt gegeben sein, wenn andere Symptome zutreffen. Die psychische Abhängigkeit steht dann im Vordergrund.

\subsubsection{Benzodiazepinabusus und Benzodiazepinabhängigkeit}

\subsubsection{Benzodiazepinabusus}

Die Übergänge zwischen Gebrauch, kritischem bzw. riskantem oder schädlichem Gebrauch, Missbrauch und einer Abhängigkeit von Medikamenten sind oftmals fließend. Wie hoch der primäre Missbrauch / Abusus von Benzodiazepinen ist, ist nicht bekannt. Im Gegensatz z.B. zur Alkoholabhängigkeit, wo es bestimmte Grenzwerte gibt.

Zum Abusus kommt es insbesondere im Zusammenhang mit einer weiteren Suchterkrankung, wie z.B. einer Alkoholabhängigkeit oder der Abhängigkeit von illegalen Drogen. Die Konsumenten nehmen Benzodiazepine ein, um die euphorisierende Wirkung einer anderen Droge zu verstärken oder um deren Entzugssymptome zu lindern. So nutzen beispielsweise heroinsüchtige Personen, die Methadon einnehmen, Diazepam oder ein anderes Derivat, um das high des Methadons zu verstärken (O’BRIEN 2005). Sie verschaffen sich die Medikamente durch den Kauf gefälschter Rezepte oder direkt von Dealern auf dem Schwarzmarkt. Die Benzodiazepine werden dabei gelegentlich auch intravenös injiziert. So kam es beispielsweise in Großbritannien zu einer weitverbeiteten intravenösen Verwendung von flüssigkeitsgefüllten Temazepamkapseln. Diese Form des Abusus betreiben jedoch nahezu ausschließlich Angehörige der illegalen Drogenszene (LAUNCHBURY et al. 1992, 
POSER W und POSER S 1996). Klinische Erfahrungen zeigten, dass Benzodiazepine mit einem raschen Wirkungseintritt ein besonders hohes Potential zum Missbrauch haben, da diese stärker euphorisierend wirken. Ebenso besteht die Gefahr des Missbrauchs bei Präparaten mit einer kurzen Halbwertszeit, da deren Einnahme häufig wiederholt werden muss, um den erwünschten Effekt zu erhalten. Diazepam z.B. ist eines der Präparate, die ein hohes Missbrauchspotential beeinhalten. Zwar handelt es sich hierbei um ein Präparat mit langer Halbwertszeit, bedingt durch aktive Metabolite, es zeichnet sich jedoch ebenfalls durch eine rasche Anflutung aus. Der Entzug von Diazepam verläuft, bedingt durch die langwirksamen aktiven Metabolite, jedoch milder als der Entzug von anderen Präparaten (O’BRIEN 2005). Es wird insbesondere auch im Benzodiazepinentzug eingesetzt. Funderburk et al. fanden in einer Studie mit ehemals benzodiazepinabhängigen Patienten eine ähnlich hohe Beliebtheit von Lorazepam, welches sich ebenfalls durch ein hohes Missbrauchspotential auszeichnet. Dieses Präparat besitzt eine relativ kurze Halbwertszeit bei intermediärer Anflutungsgeschwindigkeit (FUNDERBURK et al.1988). Neben dem Wirkungsprofil haben die Verfügbarkeit und der Preis der Benzodiazepine auf dem Schwarzmarkt Auswirkungen auf den Missbrauch der verschiedenen Präparate. Auch bei Patienten, die Benzodiazepine zunächst zu therapeutischen Zwecken erhalten, kommt es in einem geringen Anteil zum Abusus. Dies geschieht durch eine, von der ursprünglichen Verschreibung abweichenden Einnahme, die zu einer unangemessenen Steigerung der Dosis führen und letztendlich ebenfalls in der Nutzung illegaler Resourcen münden kann (O’BRIEN 2005).

\subsubsection{Benzodiazepinabhängigkeit}

Benzodiazepinabhängigkeit scheint nach Studienlage zwei gänzlich verschiedene Personengruppen zu betreffen. Auf der einen Seite Personen, die Benzodiazepine wie auch andere Suchstoffe primär aufgrund von euphorisierenden Substanzeigenschaften konsumieren, auf der anderen Seite Patienten, die primär ärztlich verordnete Dosierungen einnehmen, um Krankheitssymptome zu behandeln (O’BRIEN 2005). Letzteres betrifft den überwiegenden Teil der benzodiazepinabhängigen Personen, somit beginnt die Mehrzahl der Abhängigkeiten im Rahmen ärztlicher Behandlungen (HOLZBACH et al. 2010). Das spezielle Problem der Benzodiazepine besteht darin, dass Abhängigkeit schon im Bereich von therapeutischen Dosierungen vorkommen kann, z.B im Rahmen einer sogenannten low-dosedependency oder Niedrigdosisabhängigkeit. Diese Form der Abhängigkeit betrifft Patienten die über Jahre therapeutische Dosen von Benzodiazepinen einnehmen, ohne dass es zu einer Dosissteigerung kommt. Dennoch kann bereits eine starke psychische oder auch körperliche 
Abhängigkeit bestehen, die erst zum Zeitpunkt des Absetzversuches bemerkt wird (POSER W et al 2006). Die Wahrscheinlichkeit der Entwicklung einer Abhängigkeit durch langjährigen Benzodiazepingebrauch wird auf ca. 50\% geschätzt (BARLOW 1997, PELISSOLO et al. 2000). Die Fachinformationen der einzelnen Präparate geben daher klare Empfehlungen für die maximale Verabreichungsdauer der verschiedenen Wirkstoffe. So wird beispielsweise im Fall von Diazepam und Lorazepam eine schrittweise Verringerung der Dosis bereits nach zweiwöchiger Therapie empfohlen. Weiterhin wird im Fall des Diazepam empfohlen, eine Behandlungsdauer von 4 Wochen nicht zu überschreiten. Studien von Holzbach et al, die sich auf die Auswertung von Apothekenrezepten über einen langen Zeitraum stützen, konnten zeigen, dass die Therapie dennoch in mindestens einem Drittel der Fälle über diesen Zeitraum hinaus fortgesetzt wird. Eine problematische Dauerverschreibung findet dabei in $80 \%$ zwischen Patient und nur einem einzigen betreuenden Arzt statt. Nur ein kleiner Anteil der Patienten betreibt ein sogenanntes Ärztehopping, um sich mit den entsprechenden Rezepten zu versorgen (HOLZBACH et al. 2010).

Noch bis Ende der 90er Jahre wurde im Fall von Patienten mit chronischen Angsterkrankungen eine langzeitige Therapie mit Benzodiazepinen empfohlen. Heute spielen insbesondere neuere Antidepressiva wie z.B. die SSRIs (Selective Serotonin Wiederaufnahmehemmer) eine große Rolle und werden als Medikamente der ersten Wahl in der Behandlung von Angsterkrankungen empfohlen (ROSENBAUM 2005).

Pharmakologische Abhängigkeit ist ein physiologischer Anpassungsvorgang an die kontinuierliche Einnahme vieler Medikamente. Toleranzentwicklung tritt im Fall der kontinuierlichen Einnahme von Benzodiazepinen seltener und später auf als bei anderen Suchtstoffen wie z.B. Opioiden. Die meisten Langzeitanwender befinden sich auch nach Jahren noch im Stadium der Niedrigdosisabhängigkeit (POSER W et al. 2006). Gegenüber den sedierenden und psychomotorisch einschränkenden Wirkungen der Benzodiazepine bildet sich sicher Toleranz aus; nach O’BRIEN (2005) ist umstritten, ob dies auch betreffend die anxiolytischen Wirkung der Fall ist. Vielfach aber berichten Patienten auch über Toleranzentwicklung hinsichtlich des anxiolytischen Effektes von Benzodiazepinen. Die Verminderung der erwünschten Wirkung kann dann zu einer kontinuierlichen Dosissteigerung führen.

Im Gegensatz zur Niedrigdosisabhängigkeit besteht bei Patienten mit einer Hochdosisabhängigkeit ein höheres Krankheits- und Leidensgefühl. Durch immense Dosissteigerungen und die folgende Toleranzentwicklung geht der gewünschte Effekt der Medikation schneller verloren oder verkehrt sich sogar ins Gegenteil. Hinzu kommen oft schwere Entzugssymptome. Einer Therapie sind diese Patienten, durch den hohen Leidensdruck bedingt, 
teilweise leichter zugänglich (POSER W et al. 2006). Über die Ursache, wer eine Niedrigoder Hochdosisabhängigkeit entwickelt, ist wenig bekannt.

\subsubsection{Komorbidität der Benzodiazepinabhängigkeit}

Benzodiazepinabhängigkeit ist in hohem Maße mit dem Auftreten komorbider psychiatrischer und somatischer Erkrankungen verknüpft. Um entsprechende präventive oder therapeutische Maßnahmen ergreifen zu können, ist es wichtig, ein Profil komorbider Erkrankungen der Personen zu erstellen, die zu einem unangemessenen Gebrauch von Benzodiazepinen neigen. Unter den psychiatrischen Erkrankungen tritt eine Benzodiazepinabhängigkeit in erster Linie in Verbindung mit komorbiden Angsterkrankungen, Depressionen oder chronischen Schlafstörungen auf (MANTHEY et al. 2011). Unter den Angsterkrankungen sind dies insbesondere die Panikstörung, die generalisierte Angsterkrankung und Phobien. Auch Persönlichkeitsstörungen und somatoforme Störungsbilder werden häufig mit Benzodiazepinabhängigkeit assoziiert (MARTINEZ-CANO et al. 1999). Patienten mit einer weiteren vorbestehenden Suchterkrankung entwicklen ebenfalls überdurchschnittlich häufig eine zusätzliche Benzodiazepinabhängigkeit (LONGO und JOHNSON 2000).

Unter den rein somatischen Erkrankungen kommt Benzodiazepinabhängigkeit häufig bei Patienten mit chronischen Erkrankungen oder Schmerzsyndromen vor. Es handelt sich hierbei insbesondere um rheumatologische, neurologische oder kardiovaskuläre Erkrankungen (MARTINEZ-CANO et al. 1999, MANTHEY et al. 2011).

Weiterhin scheinen Faktoren wie Arbeitslosigkeit und das Leben als Single zu einer Einnahme von Benzodiazepinen zu prädisponieren (MANTHEY et al. 2011).

\subsubsection{Epidemiologie/ Soziodemographie von Benzodiazepinabhängigkeit}

Im Bereich der rezeptpflichtigen Arzneimittel besitzen 4-5\% ein eigenes Missbrauchs- und Abhängigkeitspotential, dabei liegen Schlaf- und Beruhigungsmittel, insbesondere aus der Klasse der Benzodiazepine, an erster Stelle. Von einer Medikamentenabhängigkeit sind in Deutschland ca. 1,4-1,9 Millionen Menschen betroffen, wovon ca. 1,1-1,2 Mio. auf Benzodiazepinderivate entfallen. Im Jahr 2008 wurden in Deutschland insgesamt 28,9 Mio. Packungen Schlaf- und Beruhigungsmittel verkauft; hiervon 59\% aus der Klasse der Benzodiazepineoder benzodiazepinähnlichen Wirkstoffe (GLAESKE 2010).

Studien aus dem Jahr 2005 zeigten, dass 11\% der US-Population im Verlauf des Jahres ein Benzodiazepinpräparat eingenommen hatte (ROSENBAUM 2005). In den letzten Jahren zeichnen sich zwei Trends ab. Auf der einen Seite eine Abnahme der Verordnungen von langwirksamen Benzodiazepinen, auf der anderen Seite eine Zunahme der Verordnungen von 
Nichtbenzodiazepinen, aber benzodiazepinagonistisch wirkenden Mitteln wie Zolpidem, Zopiclon und Zaleplon (GLAESKE 2010). Auch diese Substanzen beinhalten das Risiko von Missbrauch und Abhängigkeit, dieses scheint jedoch niedriger als im Fall der Benzodiazepine (HAJAK et al. 2003). Die Vorteile dieser Substanzen bleiben abzuwarten.

Insgesamt werden Benzodiazepine zu einem wesentlich häufigeren Anteil von ca. 70\% Frauen verordnet, wobei allerdings der Anteil eines problematischen Dauerkonsums in beiden Geschlechtergruppen ähnlich hoch ist. Auch ein höheres Alter der Patienten ist mit der erhöhten Einnahme von Benzodiazepinen verknüpft. So betrifft ein großer Anteil problematischer Dauerverschreibungen ältere Menschen. Unter den über 70 jährigen Patienten, liegt der Anteil hierbei bei 25\%, während der Anteil bei den unter 50 jährigen Patienten nur bei 10\% liegt (HOLZBACH et al. 2010).

\subsection{Benzodiazepin- und komorbide Alkoholabhängigkeit}

\subsubsection{Alkoholabhängigkeit}

\subsubsection{Definition von Alkoholabhängigkeit}

Nach den Kriterien der ICD-10 (WHO 1991) handelt es sich bei dem Alkoholabhängigkeitssyndrom um eine Gruppe körperlicher, Verhaltens-, und kognitiver Phänomene, bei denen der Konsum von Alkohol für die betreffende Person Vorrang gegenüber anderen Verhaltensweisen hat, die von ihr früher höher bewertet wurden. Entscheidend ist der starke bis übermächtige Wunsch, Alkohol zu konsumieren. Analog den Kriterien der Benzodiazepinabhängigkeit kann die Diagnose der Alkoholabhängigkeit gestellt werden, wenn während der letzten zwölf Monate mindestens drei der folgenden Kriterien gleichzeitig erfüllt waren:

1. Ein starker Wunsch oder eine Art Zwang, Alkohol zu konsumieren.

2. Verminderte Kontrollfähigkeit bezüglich des Beginns, der Beendigung und der Menge des Konsums.

3. Ein körperliches Entzugssyndrom bei Beendigung oder Reduktion des Konsums, mit substanzspezifischen Entzugssymptomen oder Ersatzkonsum, um Entzugssymptome zu mildern oder zu vermeiden.

4. Wirkungsverlust und Toleranzentwicklung, so dass höhere Mengen erforderlich sind, um die ursprüngliche Wirkung hervorzurufen.

5. Vernachlässigung anderer Interessen zugunsten des Substanzkonsums wegen erhöhtem Zeitaufwand, um die Substanz zu beschaffen, zu konsumieren oder sich von den Folgen zu erholen.

6. Anhaltender Substanzkonsum, trotz Nachweis eindeutiger schädlicher Folgen. 
Durch den allgemein akzeptierten Konsum von Alkohol beginnen der Missbrauch oder schädliche Gebrauch und der Übergang zur Alkoholabhängigkeit meist schleichend.

\subsubsection{Wirkungen des Alkoholkonsums}

Die akute Wirkung von Alkohol hängt von der aufgenommenen Trinkmenge sowie der individuellen körperlichen und seelischen Verfassung des Einzelnen ab. Generell hat Alkohol ähnlich den Benzodiazepinen anxiolytische, sedierende und hypnotische Wirkungen. Gerade die anxiolytische Wirkung scheint wesentlich zu den positiven Verstärkereigenschaften von Alkohol auf das Verhalten beizutragen (SCHMIDT et al. 2003).

Während geringe Mengen von Alkohol tendenziell anregend und stimmungssteigernd wirken, wirken höhere Mengen eher betäubend. Mit zunehmender Blutalkoholkonzentration lassen Sehvermögen, Konzentrationsfähigkeit und Bewegungskoordination nach. Ab ca. 2 Promille kommt es zu Gedächtnis- und Orientierungsstörungen. Bei Werten über drei Promille handelt es sich um eine schwere Alkoholintoxikation, die ins Koma münden oder zum Tod durch Atemstillstand führen kann (MADER 2001). Bei alkoholabhängigen Patienten können aufgrund von Toleranzentwicklung Werte von 2 oder 3 Promille aber auch gerade erst zu unauffälligem Verhalten führen. Besonders gefährlich ist die Kombination von Alkohol und Benzodiazepinen, die sich gegenseitig in ihren Wirkungen verstärken. Hierunter kann es rasch zur Entwicklung paradoxer Effekte, zu Filmrissen/ blackouts und Amnesien kommen. Dosen, die einzeln nicht tödlich gewesen wären, können hier auch zu Todesfällen führen (POSER W und POSER S 1996). Ebenso kann das plötzliche Absetzen von Alkohol zu massiven vegetativen Entzugserscheinungen bis hin zu Entzugskrampfanfällen, optischen und akustischen Halluzinationen und zum Delir führen (MADER 2001).

Die langzeitigen Folgen chronischen Alkoholgebrauchs sind multipel. Es kommt zu Zellschädigungen praktisch aller Gewebe. Besonders hervorzuheben sind hierbei Schädigungen der Leber (z.B. Leberzirrhose), des Pankreas, des Herzens (z.B. dilatative Kardiomyopathie), des zentralen und peripheren Nervensystems (z.B. Hirnatrophie oder Polyneuropathie) und der Muskulatur. Der langfristige Gebrauch führt zu einem erhöhten Krebsrisiko im gesamten Gastrointestinaltrakt. Bei Frauen steigt auch das Brustkrebsrisiko (MADER 2001).

\subsubsection{Pharmakologische Gesichtspunkte}

Neben zahlreichen weiteren pharmakologischen Wirkmechanismen wirkt Alkohol wie die Benzodiazepine auf die Aktivität der Chlorid-Kanäle des GABA-A-Rezeptors. Auch Alkohol verstärkt in einem den Benzodiazepinen ähnlichen Mechanismus die hemmende Wirkung des Neurotransmitters GABA durch Aktivierung des Rezeptorkomplexes. Dies zeigt sich auch an 
den Wirkungen der Substanzgruppen, die beide ähnlich anxiolytisch, sedierend und hypnogen wirken. Adaptive Veränderungen des Rezeptorkomplexes führen auch bei chronischem Alkoholkonsum zu Toleranzentwicklung, die sich z.B. in einer Abnahme der sedierenden Wirkung zeigt (SCHMIDT et al. 2003).

\subsubsection{Komorbide Störungsbilder}

Komorbide psychiatrische Erkrankungen, die gehäuft bei alkoholabhängigen Patienten zu finden sind, sind in erster Linie Angststörungen. Hierbei ist die Alkoholabhängigkeit häufig Folge einer Angsterkrankung, wobei Angststörungen auch durch chronischen Alkoholkonsum erzeugt werden können. Die engste Beziehung besteht zwischen Panikstörungen und Alkoholismus (SCHNEIDER et al. 2001). Auch affektive Störungsbilder, vor allem Depressionen, kommen häufig vor. Weiterhin eng mit chronischem Alkoholkonsum verknüpft sind instabile Persönlichkeitsstörungen vom Borderline-Typ, dissoziale Persönlichkeitsstörungen, Zwangsstörungen und PTBS (SCHNEIDER et al. 2001, SCHMIDT et al. 2003, DRIESSEN et al. 2008). Auch die Häufigkeit von Selbstmordversuchen oder durchgeführtem Suizid ist bei Alkoholabhängigen deutlich höher als in der Allgemeinbevölkerung (SCHMIDT et al. 2003).

\subsubsection{Epidemiologie}

Laut Suchtbericht (2009) der Drogenbeauftragten der Bundesregierung konsumieren in Deutschland 9,5 Mio. Menschen Alkohol in gesundheitlich riskanter Form. Dabei gelten ca 1,3 Mio. Menschen als abhängig. 73.000 Menschen versterben im Jahr an den Folgen des Alkoholmissbrauchs bzw. der Alkoholabhängigkeit. Pro Kopf werden jährlich ca. zehn Liter reinen Alkohols konsumiert, womit Deutschland im internationalen Vergleich im oberen Zehntel liegt.

\subsubsection{Pathogenese der Doppelabhängigkeit von Benzodiazepinen und Alkohol}

Benzodiazepinabhängigkeit und Benzodiazepinabusus treten häufig in Verbindung mit Alkoholabhängigkeit auf. Generell scheinen vorbestehende Suchterkrankungen zur Entwicklung von Medikamentenabhängigkeiten zu prädisponieren (LADER 2011). Die Gefahr der Entwicklung einer weiteren Abhängigkeit ist insbesondere dann erhöht, wenn Erststoff und Arzneimittel aus der gleichen pharmakologischen Wirkstoffgruppe kommen. So sind Heroinabhängige besonders gefährdet für den Mißbrauch bzw. die Abhängigkeit von Opioiden, Alkoholiker für den Mißbrauch bzw. die Abhängigkeit von den wirkungsverwandten Benzodiazepinen (ROSS 1993, POSER W und POSER S 1996). Bereits bei regelmäßigem 
Alkoholkonsum in moderaten Dosen wird hierbei über eine gesteigerte euphorisierende Wirkung der Benzodiazepine berichtet (DE WIT und DOTY 1994). Problematisch stellt sich dar, dass Benzodiazepine häufig in der Alkoholentgiftung eingesetzt werden. Sie sind hier wirksame Medikamente, die der Entstehung eines Entzugssyndroms einschließlich deliranter Zustände und Entzugskrampfanfällen vorbeugen können. Gerade der Einsatz von Benzodiazepinen im Rahmen dieser Indikation ist jedoch mit einem erhöhten Umsteigerisiko und demzufolge der Gefahr der Suchtentwicklung verknüpft (POSER W und POSER S 1996). In Studien konnte gezeigt werden, dass Alkoholiker, die im Entzug Benzodiazepine erhielten oder im Verlauf aufgrund von Angst- oder Schlafstörungen mit diesen weiterbehandelt wurden, eine erhöhte Prävalenz der weiteren Nutzung zeigten. Behandelnden Ärzten wird daher empfohlen, vor dem Beginn einer Therapie das Vorhandensein weiterer Suchterkrankungen genau zu überprüfen (ROSENBAUM 2005). Die American Psychiatric Association (APA 1994) gab bereits in den 1990er Jahren die Empfehlung eines vorsichtigen Einsatzes der Substanzgruppe der Benzodiazepine im Fall vorbestehender Abhängigkeiten. Die in Deutschland gängigen Fachinformationen z.B. für Diazepam, Lorazepam oder auch andere Benzodiazepine raten in diesem Fall generell vom Einsatz der Präparate ab. Ob Patienten mit psychischen Erkrankungen und komorbider Suchterkrankung überhaupt Benzodiazepine verschrieben werden sollten, wird in der Literatur kontrovers diskutiert. Eine Studie von BRUNETTE et al. (2003) zeigte, dass Benzodiazepine in einem hohen Prozentsatz auch Patienten mit komorbidem Substanzmissbrach oder Substanzabhängigkeit verschrieben wurden. Ein signifikanter Anteil von 15\% betrieb dabei einen Missbrauch. In der Studie fand sich wenig Evidenz dafür, dass diesem Patientenkollektiv durch die Therapie mit Benzodiazepinen geholfen werden konnte. Im Gegensatz zum Vergleichskollektiv ohne Benzodiazepine zeigten sich weder Zielsymptome wie Angst oder Depression deutlich gebessert, noch zeigte sich ein besseres Outcome betreffend die vorbestehenden Suchterkrankung. Es wurde somit deutlich empfohlen, diese Patienten mit anderen Substanzen zu behandeln (BRUNETTE et al. 2003).

\subsection{Entzugstherapie bei Benzodiazepinabhängigkeit}

Die Behandlung von Patienten mit einer reinen Benzodiazepinabhängigkeit gestaltet sich in einigen Fällen schwierig. Die Patienten nehmen ihre Abhängigkeit nicht als solche wahr und sind teilweise schwer zu einer Entzugsbehandlung zu motivieren. Insbesondere der stationäre Entzug zusammen mit alkoholabhängigen Patienten wird als diskriminierend empfunden und schlecht akzeptiert. Die Entzugstherapie wird von drei Determinanten wesentlich beeinflusst. Der wichtigste Parameter ist hierbei der Zeitraum, über den Benzodiazepine eingenommen 
wurden. Je länger die Einnahme erfolgte, umso wahrscheinlicher ist das Auftreten von gegebenenfalls auch schweren Entzugssymptomen. Weitere Faktoren sind die im Vorfeld eingenommene Dosis und die Halbwertszeit des eingenommenen Präparates (O’BRIEN 2005).

Der Benzodiazepinentzug kann auf verschiedene Art durchgeführt werden. In erster Linie ist eine langsame Dosisreduktion des Medikamentes wichtig, um schwere Entzugssymptome zu vermeiden. Weiterhin wird empfohlen, im Vorfeld eingenommene Benzodiazepine, auf ein langwirksames Präparat wie Diazepam umzustellen, um den Entzug zu erleichtern (VOSHAAR et al. 2006, LADER 2011). Die Dosis des im Vorfeld eingenommenen Präparates wird hierbei auf eine äquivalente Dosis Diazepam umgerechnet. Klinische Erfahrungen zeigen, daß die Anfangsstadien des Benzodiazepinentzugs von den Patienten deutlich besser toleriert werden als die letzten Reduktionsschritte (LADER 2011). Zu Beginn der Therapie erfolgt daher üblicherweise eine starke Dosisreduktion von bis zu 50\% der ursprünglich eingenommenen Dosis. Diese Startdosis wird dann an den Bedarf des Patienten und möglicherweise auftretende Entzugssymptome angepasst. Im weiteren Verlauf wird die Reduktion in kleineren Schritten fortgesetzt, dies kann entweder durch Halbierung der Dosen oder nach festen Schemata erfolgen (POSER W et al. 2006). Letztendlich gibt es keine klaren Empfehlungen zur optimalen Höhe der Dosisreduktion oder zum zeitlichen Verlauf der einzelnen Reduktionsschritte (VOSHAAR et al. 2006, LADER 2011). Es scheint vielmehr wichtig, die Geschwindigkeit der Dosisreduktion an die individuelle Entzugssymptomatik der Patienten anzupassen. Im Fall starker Entzugssymptome kann auch eine Korrektur der Reduktionsschritte mit passagerer Dosiserhöhung notwendig werden (POSER W et al. 2006). Ein großer Anteil der Patienten kann über einen Zeitraum von ca 8-12 Wochen entzogen werden (LADER 2011). In Einzelfällen wird jedoch mehr als ein Jahr für eine erfolgreiche Entzugstherapie benötigt (POSER W et al. 2006). Die Prognose bei langsamer Reduktion der Benzodiazepindosen ist gut. Bis zu zwei Drittel der Patienten schließen die Therapie ab (LADER 2011).

Im Fall von Hochdosisabhängigkeiten verläuft der Entzug prinzipiell nach dem gleichen Schema, sollte aber grundsätzlich unter stationären Bedingungen durchgeführt werden. Nicht jeder Patient mit einer Niedrigdosisabhängigkeit von Benzodiazepinen muss entzogen werden. Bisher gibt es für diese Nutzen Risiko Abwägung keine allgemein akzeptierte Vorgehensweise (POSER W et al. 2006). Auch eine Niedrigdosisabhängigkeit kann vergleichbar starke Entzugssymptome wie eine Hochdosisabhängigkeit erzeugen. So kann es z.B. zum Auftreten von Entzugskrampfanfällen kommen. Eine begleitende medikamentöse Therapie kann den Entzug erleichtern. So werden beispielsweise bei komorbiden affektiven 
Störungsbildern Antidepressiva eingesetzt (LADER 2011). Sind Entzugskrampfanfälle vorbekannt wird der Einsatz von Antikonvulsiva wie z.B. Carbamazepin empfohlen (O’BRIEN 2005). Eine unterstützende psychotherapeutische Begleitung sollte routinemäßig erfolgen (LADER 2011). Je nach individuellem Bedarf kommen hierbei gruppentherapeutische Sitzungen und kognitive oder verhaltenstherapeutische Therapien in Frage.

Im Fall von Patienten mit einer Doppelabhängigkeit von Benzodiazepinen und Alkohol erfolgt zunächst der Alkoholentzug. Waren die Benzodiazepine die in der Abhängigkeit führende Substanz, wird zunächst die im Vorfeld eingenommenene Dosis in äquivalenter Dosis eines langwirksamen Benzodiazepins weitergegeben. Die Reduktion erfolgt dann nach dem Abklingen des Alkoholentzugs. War Alkohol die führende Substanz werden die Benzodiazepine zügiger reduziert (POSER W et al. 2006).

\subsubsection{Entzugssymptome im Benzodiazepinentzug}

Der zeitliche Ablauf einer Entzugssymptomatik ist an die pharmakokinetischen Eigenschaften der verschiedenen Benzodiazepin-Derivate gekoppelt. Entzugssymptome setzen mit einer Verzögerung von ca 2-3 Halbwertszeiten des ursprünglich eingenommenen Präparates ein. Wie lange diese Symptome anhalten ist nicht voraussagbar. Sie können für Tage bis Wochen oder insbesondere in Form von psychischen Symptomen sogar für Monate fortbestehen. Die mildeste Form einer Entzugssymptomatik beschreibt das sogenannte Rebound-Phänomen, hierbei kommt es zum Wiederauftreten der ursprünglich behandelten Symptome in verstärkter Form für einen bestimmten Zeitraum (LADER 2011). Häufig auftretende Symptome des Benzodiazepinentzuges sind insbesondere psychische Symptome wie Angstzunahme, Depressionen und massive Schlafstörungen (POSER W et al. 2006, LADER 2011). RICKELS et al. (2008) beschreiben zusätzlich eine Reihe weiterer für den Benzodiazepinentzug typischer Symptome. Diese beinhalten somatische Symptome wie vermehrtes Schwitzen, Tremor, Kopfschmerzen, Myalgien, Schwindel, Müdigkeit, Schwäche, Parästhesien, Appetitverlust, Übelkeit und Durchfälle. Aber auch psychische Symptome wie vermehrte Reizbarkeit, Ruhelosigkeit, Depersonalisation, Konzentrationsstörungen und eine Verschlechterung der motorischen Koordination. Ebenfalls typisch für den Benzodiazepinentzug sind perzeptuelle Störungen wie eine verstärkte Empfindlichkeit für Töne, Gerüche und Berührungen.

Um den Schweregrad einer Entzugssymptomatik einschätzen zu können, entwickelten RICKELS et al. (2008) die Physician Withdrawal Checklist, eine einfache Selbsteinschätzungsskala für Patienten, die in 20 Items häufig beobachtete Entzugssymptome abfragt. 
In seltenen Fällen können schwere Entzugserscheinungen wie zerebrale Krampfanfälle, Delirien oder Psychosen auftreten (LAUX und König 1985, LADER 2011). Dem Auftreten typischer Entzugssymptome oder auch einem ausgeprägten Rebound-Phänomen kann durch eine langsame Dosisreduktion entgegengewirkt werden (POSER W und POSER S 1996).

\subsection{Ziel der Arbeit}

Ziel der Arbeit ist, das Verständnis der Ursachen und Einflüsse bei der Entstehung von Benzodiazepinabhängigkeit zu verbessern. Darüber hinaus sollen die negativen Folgen der Abhängigkeit bei Patienten im Benzodiazepinentzug beleuchtet werden.

Der Fokus liegt dabei auf der Erhebung der Komorbidität benzodiazepinabhängiger Patienten einerseits und auf kognitiven Einschränkungen von Patienten während der Entzugstherapie andererseits. Berücksichtigt wird der Schweregrad der ursprünglichen Abhängigkeit.

Hierbei soll ein Vergleich zwischen ausschließlich benzodiazepinabhängigen Patienten und benzodiazepin- und alkoholabhängigen Patienten erfolgen.

Insgesamt liegen bisher nur wenige Untersuchungen zur Komorbidität benzodiazepinabhängiger Patienten vor. Daher müssen zusätzliche Daten über psychiatrische und somatische Begleiterkrankungen, die mögliche Auslöser einer Benzodiazepinabhängigkeit sein können, dringend erhoben werden.

Das Ausmaß möglicher kognitiver Defizite langzeitig benzodiazepinabhängiger Patienten wird in der Literatur viel und kontrovers diskutiert. In der aktuellen Literatur gibt es nur wenige Studien zu kognitiven Defiziten speziell im Entzug. Durch spezifische Tests sollen daher mögliche Verschlechterungen kognitiver Leistungen erfasst werden.

Zusätzlich soll der Schweregrad der Abhängigkeit der Patienten untersucht und mit allen weiteren erhobenen Parametern in Zusammenhang gesetzt werden.

Benzodiazepinabhängigkeit tritt in einem hohen Ausmaß zusammen mit komorbider Alkoholabhängigkeit auf. Daher ist es wichtig zu überprüfen, worin sich Patienten mit einer Doppelabhängigkeit von Benzodiazepinen und Alkohol von rein benzodiazepinabhängigen Patienten unterscheiden.

Um diesen Fragen nachzugehen, werden im Rahmen der vorliegenden Arbeit 30 rein benzodiazepinabhängige, sowie 30 alkohol- und benzodiazepinabhängige Patienten der Klinik für Psychiatrie und Psychotherapie befragt und getestet.

Im Anschluss wird geprüft, ob sich die verschiedenen Patientenkollektive hinsichtlich vorhandener Komorbiditäten, kognitiver Funktionen und des Schweregrades der Abhängigkeit unterscheiden. Auch zu diesem Thema liegen in der Literatur nur wenige Untersuchungen vor. 


\subsubsection{Hypothesen}

\section{Hypothese 1}

Benzodiazepinabhängige Patienten unterscheiden sich von benzodiazepin- und alkoholabhängigen Patienten im Benzodiazepinentzug hinsichtlich psychiatrischer und somatischer Komorbiditäten.

\section{Hypothese 2}

Benzodiazepinabhängige Patienten haben bei Komorbidität mit einer Alkoholabhängigkeit im Benzodiazepinentzug ein höheres Ausmaß an kognitiven Defiziten als isoliert benzodiazepinabhängige Patienten.

\section{Hypothese 3}

Benzodiazepinabhängige Patienten unterschieden sich von benzodiazepin- und alkoholabhängigen Patienten im Benzodiazepinentzug hinsichtlich des Schweregrades der Abhängigkeit.

\section{Hypothese 3.1}

Ein höherer Schweregrad der Abhängigkeit beider Patientengruppen korreliert mit dem Vorhandensein von psychiatrischen und somatischen Komorbiditäten.

\section{Hypothese 3.2}

Ein höherer Schweregrad der Abhängigkeit beider Patientengruppen korreliert mit einem höheren Ausmaß an kognitiven Defiziten im Benzodiazepinentzug.

\section{Hypothese 3.3}

Ein höherer Schweregrad der Abhängigkeit korreliert mit der Anzahl bisheriger Entgiftungsbehandlungen beider Patientengruppen.

\section{Hypothese 3.4}

Das Vorhandensein psychiatrischer Komorbiditäten korreliert mit der Anzahl bisheriger Entgiftungsbehandlungen.

\section{Hypothese 3.5}

Ein höherer ASI-Score des Problembereiches „körperlicher Zustand“ (im European Addiction Severity Index (EuropASI von GSELLHOFER et al. 1999), ein Fragebogen, den wir verwendeten um den Schweregrad der Benzodiazepin- und Alkoholabhängigkeit in verschiedenen potentiellen Problembereichen zu erfassen), korreliert mit pathologischen Laborwerten, pathologischen Abdomensonographie-Befunden, cCT (kraniale Computertomographie)-Befunden, cMRT (kraniale Magnetresonanztomographie)-Befunden, EEG (Elektroenzephalographie)-Befunden oder anderen somatischen Begeleiterkrankungen. 


\section{Material und Methoden}

\subsection{Patientenrekrutierung}

\subsubsection{Auswahl der Stichprobe}

In den Jahren 2001 bis 2010 wurden insbesondere auf der Suchtstation der Klinik für Psychiatrie und Psychotherapie der Universität Göttingen 30 benzodiazepinabhängige Patienten und 30 benzodiazepin- und alkoholabhängige Patienten im Alter von 18 bis 80 Jahren zum Thema Abhängigkeit befragt und untersucht. Es handelte sich dabei um Patienten, die zur Benzodiazepinentgiftungsbehandlung aufgenommen wurden und sich zum Zeitpunkt der Datenerhebung in der Entzugsbehandlung befanden. Die zusätzlich alkoholabhängigen Patienten hatten bereits eine Alkoholentgiftung abgschlossen. Die Einwilligung für die Teilnahme an der Studie erfolgte mündlich und schriftlich durch die betreuenden Stationsärzte der Suchtstation der Psychiatrischen Klinik und auf allen übrigen Stationen. Befragt wurden die Patienten zu einem Zeitpunkt, an dem die Entgiftung schätzungsweise halb abgeschlossen war. Ermittelt wurde dieser Zeitpunkt über die Menge an Benzodiazepinen, die noch substituiert werden musste, ohne dass es zum Auftreten von starken Entzugssymptomen kam. Die Patienten wurden somit zu dem Zeitpunkt befragt, an dem die Entzugsstartdosis um die Hälfte reduziert worden war. Dieser Zeitpunkt wurde gewählt, da spätestens in der 2. Hälfte des Benzodiazepinentzuges die Motivationstherapie und weitere Therapieplanung beginnen kann und das Vorhandensein von kognitiven Defiziten hierbei sicherlich bedeutend ist. Bei allen Patienten wurde das ursprünglich eingenommene Benzodiazepin auf eine äquivalente Dosis Diazepam umgestellt und der Entzug mit Diazepam durchgeführt.

\subsubsection{Einschlusskriterien}

Um in die Studie aufgenommen werden zu können, mussten die betreffenden Patienten eine Benzodiazepinabhängigkeit oder eine Benzodiazepin- und Alkoholabhängigkeit gemäß den Leitlinien ICD 10 (WHO 1991) aufweisen und sich im stationären Benzodiazepinentzug befinden. Die untersuchten Patienten mit komorbider Alkoholabhängigkeit hatten zum Zeitpunkt der Datenerhebung eine Alkoholentgiftungsbehandlung abgeschlossen und waren alkoholabstinent. 


\subsubsection{Ausschlusskriterien}

In der Befragung wurden keine Patienten berücksichtigt, bei denen zusätzlich eine Abhängigkeit von illegalen Substanzen bestand, die zum Einschlusszeitpunkt von einer weiteren psychotropen Substanz entgifteten oder unter schweren hirnorganischen Vorschäden oder Demenzen litten. Weitere Ausschlusskriterien waren ungenügende Deutschkenntnisse sowie die gleichzeitige Teilnahme an einer anderen Studie innerhalb der letzten Wochen.

Die Patienten nahmen freiwillig nach umfassender Aufklärung an der Studie teil und erhielten keine finanzielle Aufwendung. Begleittherapien, insbesondere medikamentöse mit Einfluss auf die Kognition, wurden erfasst und in die Auswertung mit einbezogen.

\subsubsection{Ethik}

Die Studie wurde von der Ethikkommission der Georg-August-Universität Göttingen geprüft und genehmigt.

\subsection{Erhebungsinstrumente}

\subsubsection{Standardisierte Interviews}

\subsubsection{European Addiction Severity Index (ASI)}

Um den Schweregrad der Benzodiazepin- und Alkoholabhängigkeit in verschiedenen potentiellen Problembereichen zu erfassen und um einen Überblick über vorangegangene Therapien zu bekommen, wurde der European Addiction Severity Index (EuropASI von GSELLHOFER et al. 1999) erhoben. Der EuropASI ist ein semi-strukturiertes klinisches Interview, das die Möglichkeit bietet, ein vielseitiges Profil von Personen mit Suchtproblemen zu erstellen. Die Fragen betreffen sieben Lebensbereiche, die vom Substanzmissbrauch betroffen sein können: den körperlichen Zustand, Arbeits- und Unterhaltssituation, Alkoholgebrauch, Drogengebrauch, rechtliche Situation, Familie und Sozialbeziehungen sowie den psychischen Status. In jedem der sieben Bereiche wird durch eine 5-Punkte-Rating-Skala (0-4) eine Selbsteinschätzung des Patienten getroffen, in der der Schweregrad der Probleme und das Ausmaß, in dem der Patient eine Behandlung als nötig erachtet, gemessen werden. Des Weiteren wird eine Schweregrad-Einschätzung des Interviewers in einer 9-Punkte Rating-Skala (0-9) vorgenommen, die auch den weiteren Therapiebedarf einschließt. Ein Wert zwischen 0-1 entspricht keinem echten Problem und sieht somit keinen Behandlungsbedarf vor, höhere Werte zeigen ein Problem an, so dass eine 
Behandlung in Betracht gezogen werden sollte und Werte um 8-9 zeigen ein extremes Problem mit absoluter Behandlungsnotwendigkeit an. Zusätzlich dokumentiert der Interviewer, ob die erhobenen Angaben durch eine falsche Selbsteinschätzung des Patienten oder die Unfähigkeit Fragen zu verstehen, verfälscht wurden. Die Auswertung der Fragebögen erfolgte anhand eines hierzu speziell entwickelten Computerprogramms von Dietrich, Scherf-Geschke, Havemann-Reinecke und Küfner (2001 unveröff.), wobei für jeden potentiellen Problembereich Composite-Scores errechnet wurden, die den Bedarf an einer Behandlung angeben. Diese Composit-Scores basieren auf der Situation des Patienten innerhalb der letzten 30 Tage, somit sind sie für eine Erfassung der Veränderung der aktuellen Situation geeignet. Es ergaben sich Zahlenwerte von 0 (entsprechend keinem Behandlungsbedarf) bis 1 (entsprechend einem ausgeprägten Behandlungsbedarf).

Die errechneten Composit-Scores wurden nach der offiziellen Auswertungsanleitung des EuropASI wie folgt eingeteilt:

- bis 0,44 Geringer Behandlungsbedarf

- 0,45 bis 0,74 Behandlung angebracht

- 0,75 bis 1,00 Behandlung notwendig.

\subsubsection{Diagnostisches Kurzinterview bei psychischen Störungen (MINI-DIPS)}

Psychiatrische Komorbiditäten, die häufig Ausgangspunkt einer Benzodiazepinabhängigkeit sein können, wurden mit Hilfe eines strukturierten Interviews, dem Diagnostischen Kurzinterview bei psychischen Störungen (Mini-DIPS nach MARGRAF 1994), erfaßt. Es ist eine Kurzfassung des Diagnostischen Interviews bei psychischen Störungen (DIPS). Das Mini-DIPS wurde für klinisch- diagnostische Zwecke entwickelt und dient der schnellen, überblickartigen Erfassung der wichtigsten psychischen Störungen nach den Leitlinien DSMIV (APA 1994) und ICD 10 (WHO 1991). Es erlaubt eine ausreichend genaue Diagnose von Angst-, Affektiven-, Somatoformen- und Eßstörungen, sowie des Substanzabusus und von Patienten mit Psychosen. Die Fragen beziehen sich dabei auf die Situation des Patienten innerhalb der letzten 6 Monate. Eine Kurzanamnese liefert zusätzlich Soziodemographische Daten und Informationen über psychische Probleme, Medikamenten- und Alkoholmissbrauch in der Vergangenheit. Im Mini-DIPS wird durch einen Interviewleitfaden die Art und Folge der Fragen sowie die Auswertung derselben vorgegeben, der Interviewer kann jedoch bei Unklarheiten nachfragen und sein klinisches Urteil mit in die Diagnose einfließen lassen.Das Mini-DIPS gliedert die erhobenen Daten in eine primäre und in zusätzliche Diagnosen, wobei 
die primäre Diagnose die klinisch signifikantesten und schwersten Symptome des Patienten erfasst und die sekundären Diagnosen weitere leichtere psychische Störungen beschreiben.

\subsubsection{Physician Withdrawal Checklist (PWC 20)}

Die Physician Withdrawal Checklist (PWC nach RICKELS et al. 1990) wurde verwendet um Symptome zu erfassen, die häufig im Benzodiazepinentzug auftreten. Es handelt sich hierbei um eine Selbsteinschätzungsskala für Patienten, die in 20 Items häufig beobachtete Entzugssymptome abfragt. Folgende Symptome wurden hierbei abgefragt:

1. Angst- Nervosität

2. Gereiztheit

3. Dysphorische Stimmung / Depression

4. Schlaflosigkeit

5. Ermüdung- Lethargie / Energiemangel

6. Ruhelosigkeit- Erregung

7. Zittern-Zittrigkeit

8. Kopfschmerzen

9. Schwäche

10. Appetitverlust

11. Übelkeit-Erbrechen

12. Durchfälle

13. Schlechte Koordination

14. Konzentrationsschwierigkeiten-Erinnerungsprobleme

15. Muskelschmerzen-Muskelsteifheit

16. Parästhesien

17. Schwitzen

18. Schwindelgefühl-Benommenheit

19. Gesteigerte Empfindlichkeit für Geräusche, Gerüche, Berührungen oder Schmerzen

20. Depersonalisation-Derealisation.

Die Patienten sollten die Stärke der möglichen o.g. Entzugssymptome auf einer vierstufigen Skala einschätzen, die von $0=$,aktuell keine“, 1= „leichte“, 2=“mittelschwere“ bis 3= „schwere“ Symptome reichte. Berechnet wurden im Anschluss die Mittelwerte der RickelsScores der einzelnen Entzugssymptome und die Summe aller Itemscores für jeden Patienten. 


\subsubsection{Neuropsychologische Tests}

\subsubsection{Mini Mental Status Test (MMST)}

Zur orientierenden Erfassung kognitiver Defizite wurde der Mini Mental State Test (MMST nach FOLSTEIN et al. 1975) durchgeführt. Es handelt sich hierbei um einen psychometrischen Schnelltest, der eine Abschätzung verschiedener kognitiver Leistungen innerhalb von 10-15 Minuten erlaubt. Die Aufgabenstellungen des Testes überprüfen folgende Fähigkeiten: Zeitliche und örtliche Orientierung (jeweils 5 Punkte), Aufnahmefähigkeit und Gedächtnis (jeweils 3 Punkte), Aufmerksamkeit und Rechnen (5 Punkte), Sprache (3 Punkte), Lesen (1 Punkt), Schreiben (1 Punkt), konstruktive Praxis (1 Punkt) sowie das Ausführen eines Befehls (3 Punkte). Der MMST vergibt dabei maximal 30 Punkte. Vom Vorhandensein von Hirnleistungsstörungen wird bei Werten unter 25 Punkten ausgegangen (FOLSTEIN et al. 1975).

\subsubsection{Uhren-Test (Clockdrawing Test, CDT)}

Der Uhrentest (Clockdrawing Test, CDT nach SHULMAN et al. 1993) wurde zusätzlich zum MMST zur orientierenden Erfassung kognitiver Defizite verwendet. Er gilt als Screeningtest insbesondere zur Erfassung visuokonstruktiver Fähigkeiten und Handlungsplanung. Er ist kein ausreichend standardisierter Test, hat sich aber als qualitatives Messinstrument bewährt, da er sehr sensibel auf kognitive Defizite reagiert (GÜRTLER et al. 1997). Der Test ist relativ schnell und einfach umsetzbar. Die Patienten erhalten ein Blatt mit einem vorgezeichneten Kreis. Dieses „Ziffernblatt“ sollen sie mit Zahlen und Zeigern ausfüllen und eine vorgegebene Uhrzeit von 10 Minuten nach 11 Uhr einzeichnen. Für die eingezeichnete Uhr werden anschließend Scores von 1 bis 6 nach den folgenden Kriterien vergeben:

$1=$,perfekt ${ }^{\text {“ }}$

2= „leicht visuell-räumlicher Fehler“

3= „Fehlerhafte Uhrzeit bei erhaltener visuell-räumlicher Darstellung der Uhr“

4= „Mittelgradige visuell-räumliche Desorganisation, so dass ein korrektes Einzeichnen der Uhrzeit unmöglich wird“

$5=$ „Schwergradige visuell-räumliche Desorganisation“

6= „Keinerlei Darstellung einer Uhr (Ausschluß Depression/ Delir)“.

Ein Scorewert von drei oder über drei Punkten ist nach dem Uhrentest als pathologisch anzusehen. Der Test bietet die Möglichkeit, Planungsfähigkeit, numerische und visuelle Erinnerung, Verständnis, graphomotorische Fähigkeiten und visuospatiale sowie konstruktive 
Fertigkeiten des Patienten zu testen (SHULMAN 2000, KREMER 2002). Die durchschnittliche Sensitivität und Spezifität des Testes beträgt nach Shulman 85 \%.

\subsection{Daten aus Patientenakten}

Neben den o.g. psychometrischen Testverfahren erfassten wir durch Auswertung der Patientenakten zusätzliche Parameter wie:

a) Soziodemographische Gesichtspunkte:

1. Geschlecht

2. Alter

3. Partnerschaftliche Verhältnisse

4. Berufliche Situation

5. Zeitraum der Benzodiazepin- und Alkoholabhängigkeit

6. Bisherige Entgiftungsbehandlungen

7. Vor der Entzugstherapie eingenommene Wirkstoffe mit Dosierungen

b) Pharmakotherapeutische Begleitmedikation insbesonsondere Psychopharmaka:

1. Antidepressiva

2. Neuroleptika

3. Antikonvulsiva

c) Psychiatrische Komorbiditäten wie:

1. Affektive Störungen

2. Angststörungen

3. Somatoforme Störungen

4. PTBS

5. Essstörungen

6. Psychosen

d) Somatische Komorbiditäten wie:

1. Erkrankungen des Bewegungsapparates

2. Chronische Schmerzsyndrome

3. Neurologische Erkrankungen

4. Erkrankungen des Herz-Kreislauf-Systems

5. Stoffwechselerkrankungen 
e) Laborwerte wie:

1. Leberwerte

2. Harnsäure

f) Bildgebende Befunde wie:

1. Sono Abdomen

2. CCT und cMRT Befunde

g) EEG-Befunde.

\subsection{Statistische Datenanalyse}

Alle Datenauswertungen wurden mit SPSS 18.0 und Microsoft Excel 2007 durchgeführt.

Im Fall ordinalskalierter Daten (z.B. ASI-Scores) wurden die entsprechenden nichtparametrischen Verfahren Mann-Whitney-U-Test, Spearman-Korrelationen verwendet. Diese nichtparametrischen Tests wurden auch bei metrischen Daten verwendet, da auf Grund der Spezifität der Stichprobe die Voraussetzungen zur Verwendung parametrischer Verfahren (Normalverteilung) nicht immer gegeben waren. Dichotome Daten wurden mit Hilfe des ChiQuadrat-Tests ausgewertet. 


\section{Ergebnisse}

\subsection{Soziodemographische Gesichtspunkte}

\subsubsection{Patientenkollektiv}

60 Patienten der Klinik für Psychiatrie und Psychotherapie der Universität Göttingen in aktueller stationärer Behandlung konnten nach Überprüfung der Ein- und Ausschlusskriterien in die Studie eingeschlossen werden. Es handelte sich dabei um Teilnehmer, die zur Benzodiazepin-Entgiftungsbehandlung aufgenommen wurden und sich zum Zeitpunkt der Datenerhebung in der Entzugsbehandlung befanden. Um einen Vergleich zwischen den verschiedenen Patientengruppen durchführen zu können, wurden 30 Patienten mit isolierter Benzodiazepinabhängigkeit und 30 Patienten mit einer Doppelabhängigkeit von Benzodiazepinen und Alkohol eingeschlossen. Die Einwilligung für die Teilnahme an der Studie erfolgte mündlich und schriftlich durch die betreuenden Stationsärzte der Suchtstation der Psychiatrischen Klinik und auf allen übrigen Stationen. Befragt wurden alle Patienten zu einem Zeitpunkt, an dem die Entgiftung schätzungsweise halb abgeschlossen war. Dieser Zeitpunkt wurde gewählt, da spätestens in der 2. Hälfte des Benzodiazepinentzuges die Motivationstherapie und weitere Therapieplanungen beginnen und das Vorhandensein von kognitiven Defiziten hierbei sicherlich bedeutend ist. Ermittelt wurde dieser Zeitpunkt über die Menge an Benzodiazepinen, die noch substituiert werden musste. Die Patienten wurden somit zu dem Zeitpunkt befragt, an dem die Entzugsstartdosis um die Hälfte reduziert worden war. Bei allen Patienten wurde das ursprünglich eingenommene Benzodiazepin auf eine äquivalente Dosis Diazepam umgestellt und der Entzug im Weiteren mit Diazepam durchgeführt. Im weiteren Text wird die Gruppe der rein benzodiazepinabhängigen Patienten als Gruppe A, die der Benzodiazepin- und Alkoholabhängigen Patienten als Gruppe B benannt.

\subsubsection{Geschlecht}

In der gesamten Studiengruppe befanden sich 46,7\% Frauen ( $\mathrm{n}=28)$ und 53,3\% Männer $(\mathrm{n}=32)$. In Gruppe A als Gruppe der rein benzodiazepinabhängigen Patienten befanden sich 56,6\% Frauen ( $n=17)$ und 43,3\% Männer ( $n=13)$. In Gruppe B als Gruppe der benzodiazepinund alkoholabhängigen Patienten befanden sich 36,7\% Frauen (n=11) und 63,3\% Männer $(n=19)$. 


\subsubsection{Altersverteilung}

Das Durchschnittsalter der gesamten Studiengruppe lag bei 51,7 Jahren (SD 12,5; Median 53; Spanne 21-78). Dabei lag das mittlere Alter in Gruppe A bei 53,7 Jahren (SD 14; Median 56,5). Das mittlere Alter in Gruppe B bei 49,6 Jahren (SD 10,6; Median 51). Somit ergaben sich keine signifikanten Gruppenunterschiede.

\subsubsection{Partnerschaft und Berufstätigkeit}

Bei Betrachtung der partnerschaftlichen Beziehungen zeigte sich, dass $40 \%$ der Teilnehmer verheiratet waren. 30\% der Teilnehmer waren ledig geblieben und ca 22\% geschieden. Der Anteill lediger und auch geschiedener Probanden lag in der Gruppe B, als Gruppe der zusätzlich alkoholabhängigen Patienten, höher. Der Anteil verheirateter Teilnehmer betrug hier 36,7\%, der Anteil an ledigen Teilnehmern 33,3\% und die Scheidungsrate lag bei 23,3\%. Die erhobenen Daten werden in Tabelle 1 zusammengefasst.

Tabelle 1: Partnerschaft und berufliche Situation der gesamten Studiengruppe (n=60), in Gruppe A $(n=30)$ und Gruppe B (n=30). Angabe in Prozent und Anzahl der Patienten.

\begin{tabular}{|c|c|c|c|c|c|c|}
\hline & \multicolumn{2}{|c|}{$\begin{array}{l}\text { Gesamte Studiengruppe } \\
\qquad(n=60)\end{array}$} & \multicolumn{2}{|c|}{$\begin{array}{c}\text { Gruppe A } \\
(n=30)\end{array}$} & \multicolumn{2}{|c|}{$\begin{array}{l}\text { Gruppe B } \\
(n=30)\end{array}$} \\
\hline & Prozent & Anzahl & Prozent & Anzahl & Prozent & Anzahl \\
\hline \multicolumn{7}{|l|}{ Partnerschaft } \\
\hline verheiratet & 40 & 24 & 43,3 & 13 & 36,7 & 11 \\
\hline geschieden & 21,7 & 13 & 20,0 & 6 & 23,3 & 7 \\
\hline ledig & 30 & 18 & 26,7 & 8 & 33,3 & 10 \\
\hline verwitwet & 5 & 3 & 6,7 & 2 & 3,3 & 1 \\
\hline getrennt lebend & 3,3 & 2 & 3,3 & 1 & 3,3 & 1 \\
\hline \multicolumn{7}{|l|}{ Berufstätigkeit } \\
\hline berufstätig & 28,3 & 17 & 26,7 & 8 & 30,0 & 9 \\
\hline arbeitslos & 26,7 & 16 & 23,3 & 7 & 30,0 & 9 \\
\hline Rentner & 36,7 & 22 & 40,0 & 12 & 33,3 & 10 \\
\hline Hausfrau & 8,3 & 5 & 10,0 & 3 & 6,7 & 2 \\
\hline \multicolumn{7}{|c|}{ Medizinische Berufe } \\
\hline $\begin{array}{l}\text { Angehörige med. } \\
\text { Berufsgruppen }\end{array}$ & 33 & 20 & 33 & 10 & 33 & 10 \\
\hline
\end{tabular}

In der gesamten Studiengruppe waren 26,7\% der Patienten arbeitslos; nur ungefähr ein Drittel befand sich überhaupt in einem geregelten Arbeitsverhältnis. Auffallend groß war das Quantum der Patienten, die vollständig aus dem Arbeitsleben ausgeschieden waren (Rentner und Frührentner), insbesondere in Bezug auf den Altersdurchschnitt. Weiterhin auffallend hoch stellte sich der Anteil an Mitarbeitern aus medizinischen Berufen dar, der bei 33\% lag. Signifikante Gruppenunterschiede ergaben sich die berufliche Situation betreffend nicht. 


\subsubsection{Zeitraum der Abhängigkeit}

\subsubsection{Zeitraum der Benzodiazepinabhängigkeit}

Der Einnahmezeitraum beschreibt den anamnestisch erhobenen Zeitraum der Benzodiazepineinnahme in Jahren bis zum Entzugsstart. Der mittlere Wert des gesamten Kollektivs lag bei 10 Jahren (SD 15,7; Median 6; Spanne 1-43). Die mittlere Einnahmedauer in Gruppe A lag bei 8,62 Jahren (SD 8,5; Median 6). Die mittlere Einnahmedauer in Gruppe B bei 11,5 Jahren (SD 10,8; Median 8). Statistisch signifikante Unterschiede zwischen den Gruppen bestanden nicht.

\subsubsection{Zeitraum der Alkoholabhängigkeit}

In der Gruppe der doppelabhängigen Patienten dokumentierten wir den abhängigen Alkoholkonsum in Jahren. Definiert wurde die Alkoholabhängigkeit dabei nach den Kriterien der ICD 10 (siehe oben). Der mittlere Wert an Jahren, in denen regelmäßig abhängig getrunken wurde, lag bei 14,2 Jahren (SD 8,6; Median 8).

\subsubsection{Entgiftungsbehandlungen}

Als weiteren Parameter dokumentierten wir die Anzahl der im Vorfeld der aktuellen Entzugsbehandlung stattgehabten Entgiftungen. Im gesamten Kollektiv betrug die Anzahl der stattgefundenen Entgiftungen im Mittel 4,1 (SD 7; Median 2; Spanne 0-50). In Gruppe A lag sie im Mittel bei 1,6 Entgiftungen (SD 1,5; Median 1). In der Gruppe B bei 6,6 Entgiftungen (SD 9,3, Median 4,5). Die stark erhöhten Werte der doppelabhängigen Patienten scheinen verständlich unter Berücksichtigung der in diesem Kollektiv stattgehabten multiplen Alkoholentzüge.

\subsubsection{Benzodiazepindosis vor der Entzugstherapie}

Die Patienten wurden nach der zu Hause eingenommenen Benzodiazepinmenge befragt. Da teilweise mehrere verschiedene Präparate eingenommen wurden, sind die verschiedenen Dosen der Präparate auf eine Äquivalenzdosis Diazepam umgerechnet worden. Das gesamte Patientenkollektiv nahm laut eigenen Angaben vor dem Entzug im Mittel täglich eine Diazepamäquivalenzdosis von 35,7 mg ein (SD 30; Median 28,5; Spanne 4-167). In Gruppe A lagen die Werte dabei bei im Mittel bei einer Äquivalenzdosis von 38,3 mg (SD 33,8; Median 27,5). In Gruppe B im Mittel bei einer Äquivalenzdosis von 33,0 mg (SD 26,2; Median 28,5). Die Gruppen unterschieden sich nicht signifikant ( $p=0,43$; n.s.) in der Menge der vor der Entzugstherapie eingenommen Benzodiazepine. Erfahrungen der psychiatrischen 
Kollegen zeigten, dass die Werte jedoch nur eingeschränkt beurteilbar sind, da Entzugspatienten oftmals zu hohe Dosen angeben, um zum Entzugsbeginn höhere Substitutionsdosen zu erhalten. Andere Patienten dagegen geben niedrigere Werte an, um die Stärke der Abhängigkeit zu bagatellisieren. Wir dokumentierten daher zusätzlich die tatsächliche Entzugsstartdosis.

\subsubsection{Entzugsstartdosis}

Bei der Entzugsstartdosis handelt es sich um den Wert der täglichen Diazepamdosis, die vom Patienten nach 1-3 Tagen Entzug toleriert wurde, ohne dass es zur Entwicklung starker Entzugssymptome kam. Begonnen wurde der Benzodiazepinentzug dabei meist mit etwa der Hälfte der Menge des zu Hause eingenommen Benzodiazepins, umgerechnet in eine Äquivalenzdosis Diazepam. Diese Dosis wurde je nach Verträglichkeit in den ersten drei Tagen nach oben oder unten korrigiert. Die mittlere Entzugsstartdosis des Gesamtkollektivs lag bei einer Äquivalenzdosis von 22,6 mg Diazepam pro Tag (SD 17,3; Median 20; Spanne 0-70). Die mittlere Entzugsstartdosis in Gruppe A lag bei einer Äquivalenzdosis von 24,2 mg Diazepam pro Tag (SD 19,1; Median 22,5). Die mittlere Dosis in Gruppe B bei einer Äquivalenzdosis von 20,9 mg Diazepam pro Tag (SD 15,7; Median 20).

\subsubsection{Wirkstoffe}

Die Teilnehmer nahmen im Vorfeld verschiedenste Wirkstoffe ein. Teilweise wurden auch mehrere Präparate gleichzeitig konsumiert. Tabelle 2 zeigt eine Auflistung der Wirkstoffe, die vor der Entzugstherapie eingenommen wurden.

Tabelle 2: Vor der Entzugstherapie eingenommene Benzodiazepine. Auflistung der verschiedenen Wirkstoffe und Anzahl der Teilnehmer, die diese konsumierten

\begin{tabular}{|l|c|}
\hline Im Vorfeld eingenommene Wirkstoffe & Gesamte Studiengruppe $(\mathbf{n = 6 0})$ \\
\hline & Anzahl der Teilnehmer \\
\hline Diazepam & 34 \\
\hline Lorazepam & 10 \\
\hline Bromazepam & $\mathbf{8}$ \\
\hline Lormetazepam & 6 \\
\hline Clonazepam & 5 \\
\hline Oxazepam & 4 \\
\hline Alprzolam & 2 \\
\hline Tetrazepam & 2 \\
\hline Temazepam & 1 \\
\hline
\end{tabular}


Der am häufigsten im Vorfeld eingenommene Wirkstoff war Diazepam. Mehr als die Hälfte (57\%) der Patienten nahm ein Präparat ein, das diesen Wirkstoff beinhaltete. Auch Lorazepam wurde häufig verwendet. So konsumierten ca. 17\% der Studienteilnehmer im Vorfeld lorazepamhaltige Präparate. Von 12 Teilnehmern wurden mehrere verschiedene Wirkstoffe gleichzeitig eingenommen.

\subsubsection{Zusammenfassung der Patientencharakteristika}

Tabelle 3 fasst alle Patientencharakteristika zusammen.

Tabelle 3: Zusammenfassung der Patientencharakteristika im gesamten Kollektiv ( $n=60)$, in Gruppe A $(n=30)$ und Gruppe $B(n=30)$

\begin{tabular}{|c|c|c|c|c|c|c|}
\hline \multirow[b]{2}{*}{ Geschlecht } & \multicolumn{2}{|c|}{$\begin{array}{l}\text { Gesamte Studiengruppe } \\
\qquad(\mathrm{n}=60)\end{array}$} & \multicolumn{2}{|c|}{$\begin{array}{c}\text { Gruppe } A \\
(n=30)\end{array}$} & \multicolumn{2}{|c|}{$\begin{array}{c}\text { Gruppe B } \\
(n=30)\end{array}$} \\
\hline & Mittelwert & Median & Mittelwert & Median & Mittelwert & Median \\
\hline Frauen (\%) & 46,7 & & 56,6 & & 36,7 & \\
\hline Männer (\%) & 53,3 & & 43,3 & & 63,3 & \\
\hline \multicolumn{7}{|l|}{ Alter } \\
\hline Angabe in Jahren & 51,7 & 53 & 53,7 & 56 & 49,6 & 51 \\
\hline \multicolumn{7}{|l|}{ Partnerschaft } \\
\hline verheiratet (\%) & 40,0 & & 43,3 & & 36,7 & \\
\hline geschieden (\%) & 21,7 & & 20,0 & & 23,3 & \\
\hline ledig (\%) & 30,0 & & 26,7 & & 33,3 & \\
\hline verwitwet (\%) & 5,0 & & 6,7 & & 3,3 & \\
\hline getrennt lebend (\%) & 3,3 & & 3,3 & & 3,3 & \\
\hline \multicolumn{7}{|l|}{ Berufstätigkeit } \\
\hline berufstätig (\%) & 28,3 & & 26,7 & & 30,0 & \\
\hline arbeitslos (\%) & 26,6 & & 23,3 & & 30,0 & \\
\hline Rentner (\%) & 36,7 & & 40,0 & & 33,3 & \\
\hline Hausfrau (\%) & 8,3 & & 10,0 & & 6,7 & \\
\hline \multicolumn{7}{|c|}{ Einnahmezeitraum der Benzodiazepine } \\
\hline Angabe in Jahren & 10,0 & 6 & 8,6 & 6 & 11,5 & 8 \\
\hline \multicolumn{7}{|l|}{ Alkoholkonsum } \\
\hline Angabe in Jahren & & & 0 & 0 & 14,2 & 11 \\
\hline \multicolumn{7}{|c|}{ Anamnestisch eingenommene Benzodiazepinmenge } \\
\hline $\begin{array}{l}\text { Äquivalenzdosis } \\
\text { Diazepam (in mg) }\end{array}$ & 35,7 & 28 & 38,3 & 27 & 33,0 & 28 \\
\hline \multicolumn{7}{|l|}{ Entzugsstartdosis } \\
\hline Äquivalenzdosis & 26 & O & $21 ?$ & 22 & 210 & 20 \\
\hline \multicolumn{7}{|c|}{ Anzahl bisheriger Entgiftungen } \\
\hline Anzahl & 4,1 & 2 & 1,6 & 1,0 & 6,5 & \\
\hline
\end{tabular}

\subsection{Pharmakotherapie in der Entzugsbehandlung}

Aus den Patientenakten entnahmen wir die Begleitmedikation während des Entzugs zum Zeitpunkt der Befragung. Graphik 1 zeigt die Verteilung der verschiedenen Substanzgruppen. 


\section{Begleitmedikation}

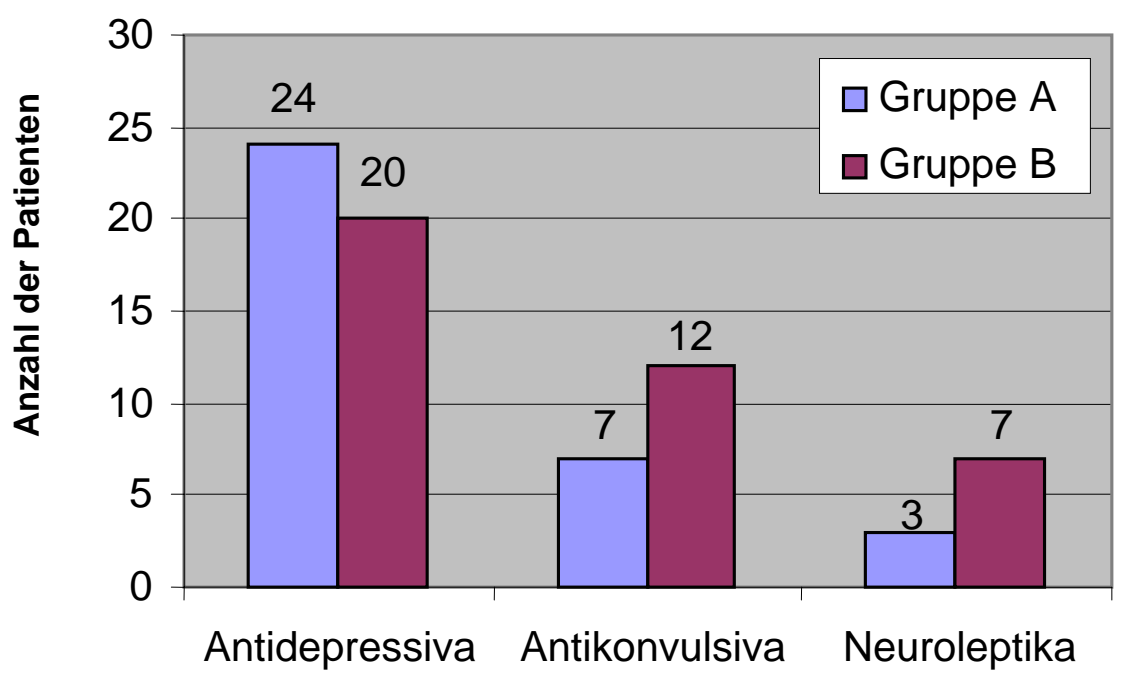

Graphik 1: Verteilung der verschiedenen Substanzgruppen von Psychopharmaka in Gruppe A $(n=29)$ und Gruppe B (n=30) zum Zeitpunkt der Befragung. Angabe in Anzahl der Patienten.

Im Folgenden werden die am häufigsten vorhandenen Wirkstoffe der verwendeten Psychopharmaka in Prozent der Patienten bzw. Anzahl der Patienten angegeben. Als weiterer Parameter wird die Durchschnittsdosis angegeben (vgl. Tabelle 4).

Tabelle 4: Am häufigsten im Entzug verwendete Psychopharmaka in Gruppe A $(n=29)$ und Gruppe B $(\mathbf{n}=30)$. Angaben in Anzahl und Prozent der Patienten sowie Angabe der Durchschnittsdosis des Medikamentes zum Zeitpunkt der Befragung.

\begin{tabular}{|c|c|c|c|c|c|}
\hline & \multicolumn{2}{|c|}{$\begin{array}{c}\text { Gruppe A } \\
(n=29)\end{array}$} & \multicolumn{2}{|c|}{$\begin{array}{c}\text { Gruppe B } \\
(n=30)\end{array}$} & \multirow{2}{*}{$\begin{array}{c}\text { Durschnittsdosis } \\
(\mathrm{mg})\end{array}$} \\
\hline & (n) & $(\%)$ & (n) & $(\%)$ & \\
\hline \multicolumn{6}{|l|}{ Antidepressiva } \\
\hline \multicolumn{6}{|c|}{$\begin{array}{l}\text { Tri-l Tetrazyklische } \\
\text { Antidepressiva }\end{array}$} \\
\hline Trimipramin & 4 & 13,8 & 8 & 26,7 & 156,8 \\
\hline Doxepin & 12 & 41,4 & 6 & 20,0 & 59,1 \\
\hline \multicolumn{6}{|c|}{ Atypische Antidepressiva } \\
\hline Mirtazapin & 9 & 31,0 & 3 & 10,0 & 33,2 \\
\hline \multicolumn{6}{|l|}{ SSRI } \\
\hline Citalopram & 4 & 13,8 & 2 & 6,7 & 21,7 \\
\hline \multicolumn{6}{|c|}{ Antikonvulsiva } \\
\hline Carbamazepin & 6 & 20,7 & 11 & 36,7 & 522,9 \\
\hline \multicolumn{6}{|l|}{ Neuroleptika } \\
\hline Quetiapin & 3 & 10,3 & 4 & 13,3 & 175,0 \\
\hline
\end{tabular}


Der Kommentar zu den verabreichten Wirkstoffen beschränkt sich zur Vereinfachung auf die Häufigkeit des Einsatzes der einzelnen Substanzen. Anmerkungen zu den Dosierungen erscheinen schwierig, da es sich bei den ermittelten Werten um Durchschnittsdosen handelt, die durch Ein- und Ausschleichen der Medikamente nicht die tatsächliche therapeutische Dosis widerspiegeln können. Medikamentenspiegel lagen nur in wenigen Fällen vor. Während der stationären Entzugsbehandlung erhielt ein Großteil der Patienten eine begleitende antidpressive Medikation. In Gruppe A wurden 82,8\% der Patienten unterstützend antidepressiv behandelt, in Gruppe B 66,7\%. Verwendet wurde in ca. einem Drittel der Fälle das trizyklische Antidepressivum Doxepin (30,7\%) ein weiterer großer Anteil der Patienten erhielt Trimipramin (20,2\%). Aus der Gruppe der atypischen Antidepressiva wurde Mirtazapin (20,5\%) am häufigsten verabreicht. Unter den SSRIs wurde vorwiegend Citalopram (10,2\%) verordnet. Zur Vorbeugung von Entzugskrampfanfällen wurden $40 \%$ der Patienten aus Gruppe B und 24,1\% der Patienten aus Gruppe A antikonvulsiv behandelt. Die Patienten erhielten dabei in erster Linie Carbamazepin (28,7\%). Ca. 17\% der Patienten wurden mit einem Neuroleptikum behandelt, wobei vorwiegend Quetiapin $(11,9 \%)$ gegeben wurde.

\subsection{Komorbide psychiatrische und somatische Erkrankungen}

\subsubsection{Psychiatrische komorbide Störungsbilder}

Psychiatrische komorbide Störungsbilder wurden mit Hilfe eines strukturierten Interviews, dem Diagnostischen Kurzinterview bei psychischen Störungen Mini-DIPS (nach MARGRAF 1994) erfasst. Es erlaubt eine ausreichend genaue Diagnose von Angst-, Affektiven-, Somatoformen- und Essstörungen; weiterhin werden Substanzabusus und Psychosen miterfasst. Zusätzliche Daten wurden durch Aktenrecherche erhoben.

Ein hoher Prozentsatz der Patienten wies zusätzliche psychiatrische Störungen auf. Die folgende Graphik 2 zeigt die Verteilung psychischer komorbider Störungsbilder angegeben in Prozent der Patienten. 


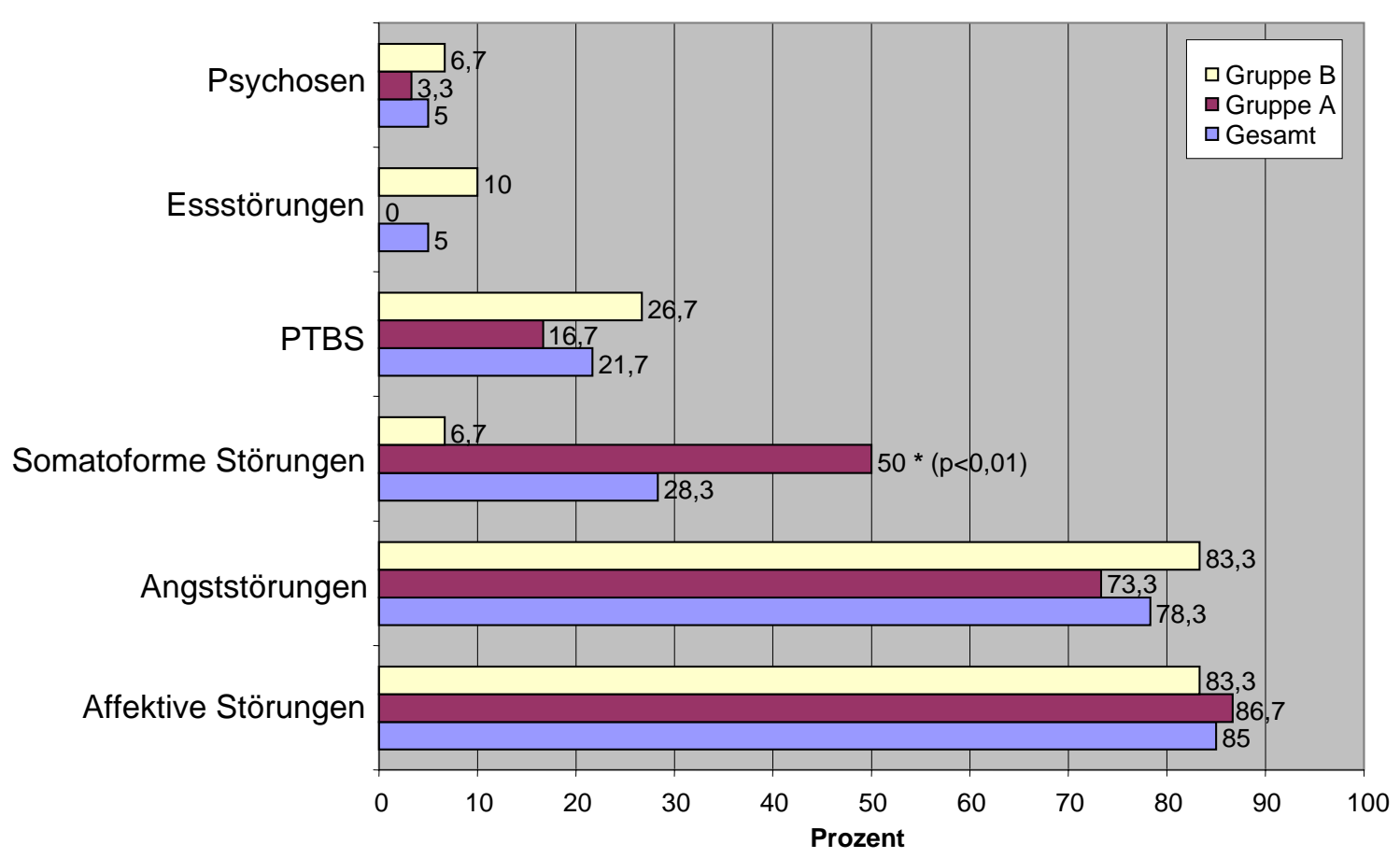

Graphik 2: Verteilung (in \%) komorbider psychischer Störungen im gesamten Patientenkollektiv (n=60), in Gruppe A $(n=30)$ und Gruppe B $(n=30)$. Im Vergleich der Gruppen mittels des Chi-Quadrat-Tests ergaben sich signifikante Unterschiede lediglich in der Häufigkeit des Auftretens somatoformer Störungsbilder, die in Gruppe A signfikant häufiger auftraten $(\mathbf{p}<0,01)$.

Ein Großteil dieser Störungen ist allerdings als wesentlicher Ausgangspunkt bzw. Ursache der Benzodiazepinabhängigkeit zu sehen. Im Vordergrund standen affektive Störungen, die von 85\% der Patienten beschrieben wurden. Mit einem Anteil von 80\% waren dies in erster Linie depressive Störungsbilder. Einen weiteren großen Anteil machten Angststörungen aus, die von 78\% der Patienten beschrieben wurden. Es handelte sich hierbei zu 55\% um Panikstörungen. Beide Störungsbilder wiesen im Gruppenvergleich eine ähnliche Verteilung auf. 28\% der Patienten erfüllten die Kriterien für eine somatoforme Störung, wobei 21\% auf Somatisierungsstörungen entfielen und 15\% auf Schmerzsyndrome. Im Vergleich der Gruppen zeigte sich ein wesentlich häufigeres Auftreten der Störungen in Gruppe A. Eine PTBS (Posttraumatische Belastungsstörung) wurde von 21\% der Patienten beschrieben mit ebenfalls ähnlicher Verteilung in den Gruppen. Nur selten traten Essstörungen auf, die 5\% der Patienten beschrieben und Psychosen, die ebenfalls von 5\% der Patienten beschrieben wurden. Die folgende Tabelle zeigt eine Untergliederung der psychiatrischen komorbiden Störungsbilder. 
Tabelle 5: Komorbide psychische Erkrankungen des gesamten Kollektivs ( $n=60)$, in Gruppe $A(n=30)$ und in Gruppe B $(n=30)$. Angabe in Prozent. Im Vergleich der Gruppen mittels des Chi-Quadrat-Tests ergaben sich signifikante Unterschiede * lediglich in der Häufigkeit des Auftretens somatoformer Störungsbilder, die in Gruppa A signifikant häufiger auftraten $(\mathbf{p}<0,01)$.

\begin{tabular}{|l|r|r|r|}
\hline & $\begin{array}{l}\text { Gesamte Studiengruppe (n=60) } \\
\text { Angaben in Prozent }\end{array}$ & $\begin{array}{l}\text { Gruppe A (n=30) } \\
\text { Angaben in Prozent }\end{array}$ & $\begin{array}{l}\text { Gruppe B (n=30) } \\
\text { Angaben in Prozent }\end{array}$ \\
\hline Affektive Störungen & $\mathbf{8 5 , 0}$ & $\mathbf{8 6 , 7}$ & $\mathbf{8 3 , 3}$ \\
\hline Depressionen & 80,0 & 86,6 & 73,3 \\
\hline Bipolare Störungen & 5,0 & 0 & 10,0 \\
\hline Angststörungen & $\mathbf{7 8 , 3}$ & $\mathbf{7 3 , 3}$ & $\mathbf{8 3 , 3}$ \\
\hline Panikstörung & 56,7 & 60,0 & 53,3 \\
\hline Agoraphobie & 36,7 & 40,0 & 33,3 \\
\hline GAS & 25,0 & 30,0 & 20,0 \\
\hline Spezifische Phobien & 15,0 & 10,0 & 20,0 \\
\hline Sozialphobie & 11,7 & 10,0 & 13,3 \\
\hline Somatoforme Störungen * & $\mathbf{2 8 , 3}$ & $* 50,0$ & $\mathbf{6 , 7}$ \\
\hline Somatisierungsstörung & 21,7 & 40,0 & 3,3 \\
\hline Schmerzsyndrom & 15,0 & 26,7 & 3,3 \\
\hline PTBS & $\mathbf{2 1 , 7}$ & $\mathbf{1 6 , 7}$ & $\mathbf{2 6 , 7}$ \\
\hline Essstörungen & $\mathbf{5 , 0}$ & $\mathbf{0}$ & $\mathbf{1 0 , 0}$ \\
\hline Psychosen & $\mathbf{5 , 0}$ & $\mathbf{3 , 3}$ & $\mathbf{6 , 7}$ \\
\hline
\end{tabular}

\subsubsection{Somatische komorbide Störungsbilder}

\subsubsection{Begleiterkrankungen}

Somatische Komorbiditäten wurden durch Aktenrecherche und Befragung der Patienten erfasst. In einem hohen Prozentsatz der Studiengruppe fanden sich somatische Komorbiditäten. Wir dokumentierten Störungen des Bewegungsapparates, chronische Schmerzsyndrome und neurologische Erkrankungen als organische Erkrankungen, deren medikamentöse Behandlung sekundär zu einer Benzodiazepinabhängigkeit führen kann. Weiterhin wurden Erkrankungen des Herz-Kreislauf Systems und Stoffwechselerkrankungen erfasst (vgl. Graphik 3).

Erkrankungen des Bewegungsapparates traten mit einem Anteil von 48\% Prozent der Patienten häufig auf. Es handelte sich hierbei mit 43\% in erster Linie um degenerative Veränderungen des Bewegungsapparates. Im Vergleich der Gruppen zeigte sich ein häufigeres Auftreten in Gruppe A. Einen weiteren großen Anteil machten chronische Schmerzsyndrome aus, die von 45\% der Patienten beschrieben wurden. In erster Linie bedingt durch muskuloskelettale Probleme. Im Vergleich zwischen den Gruppen zeigte sich auch hier ein höherer Anteil in Gruppe A. Neurologische Erkrankungen traten in 23\% der Fälle auf. HerzKreislauf-Erkrankungen in 33\%. Wobei 53\% der Patienten einen arteriellen Hypertonus aufwiesen. Stoffwechselerkrankungen zeigten 25\% der Patienten. Beide Gruppen wiesen hierbei eine ähnliche Verteilung auf. In der folgenden Tabelle 6 werden die somatischen Störungen in ihrer Untergliederung aufgelistet. 


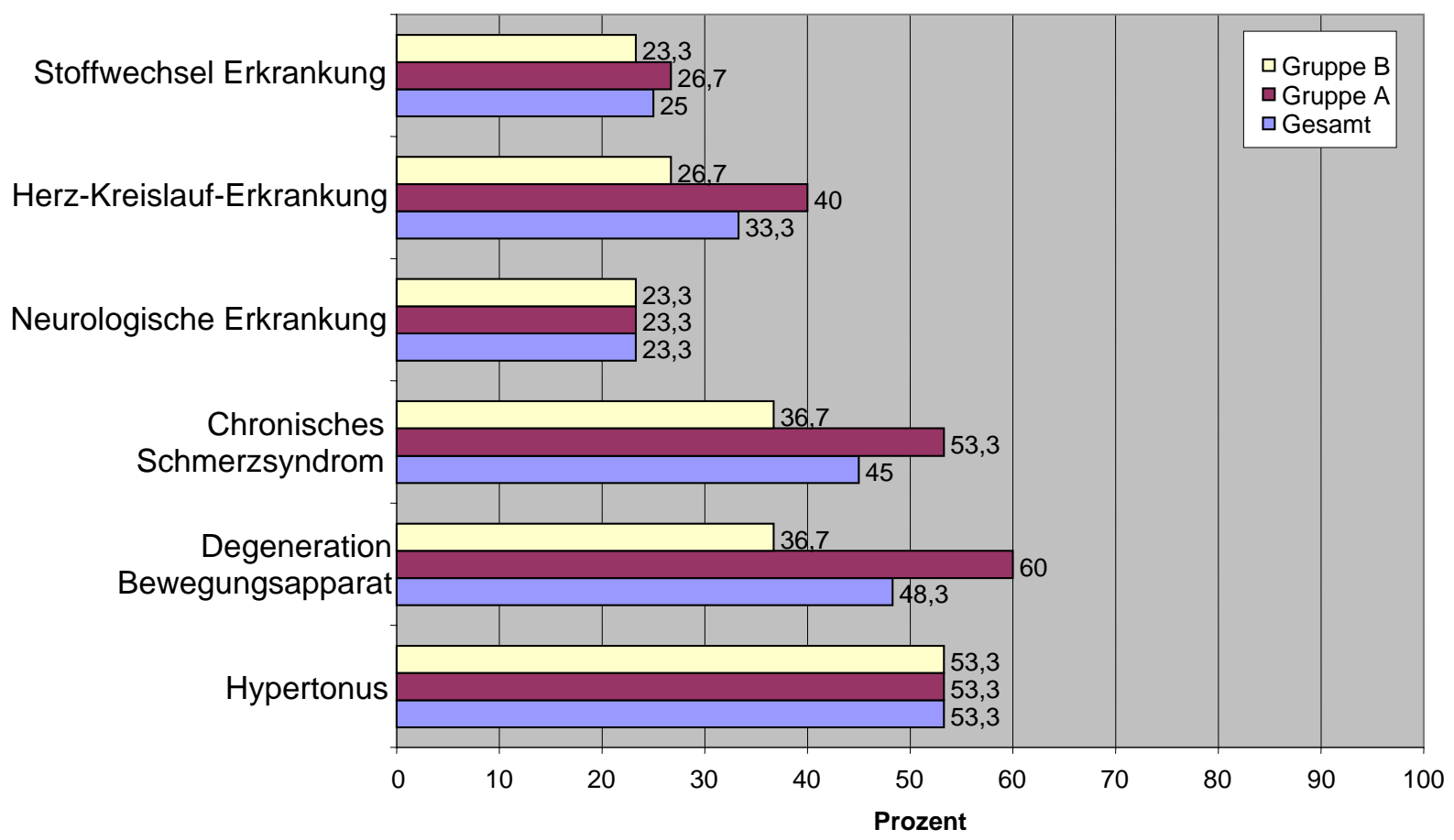

Graphik 3: Abbildung somatische Begleiterkrankungen im gesamten Kollektiv(n=60), in Gruppe A $(n=30)$ und Gruppe B $(n=30)$. Im Vergleich der Gruppen mittels des Chi-Quadrat-Tests ergaben sich keine signifikanten Unterschiede zwischen den Gruppen.

Tabelle 6: Komorbide somatische Erkrankungen des gesamten Kollektivs $(n=60)$, in Gruppe A $(n=30)$ und in Gruppe B $(n=30)$. Angabe in Prozent. Im Vergleich der Gruppen mittels des Chi-Quadrat-Test ergaben sich keine signifikanten Unterschiede zwischen den Gruppen.

\begin{tabular}{|c|c|c|c|}
\hline & $\begin{array}{l}\text { Gesamte } \\
\text { Studiengruppe }(n=30)\end{array}$ & $\begin{array}{l}\text { Gruppe A } \\
(n=30)\end{array}$ & $\begin{array}{l}\text { Gruppe B } \\
(n=30)\end{array}$ \\
\hline & Angaben in Prozent & Angaben in Prozent & Angaben in Prozent \\
\hline Erkrankungen des Bewegungsapparates & 48,3 & 60,0 & 36,7 \\
\hline Degenerative Veränderungen & 43,3 & 56,7 & 30,0 \\
\hline Bandscheibenprolaps & 15,0 & 20,0 & 10,0 \\
\hline Rheumatische Erkrankungen & 11,7 & 16,7 & 6,6 \\
\hline Chronisches Schmerzsyndrom & 45,0 & 53,3 & 36,7 \\
\hline Muskuloskelettaler Schmerz & 45,0 & 56,7 & 33,3 \\
\hline Weichteilschmerzen & 11,7 & 13,3 & 10,0 \\
\hline Cephalgien & 5,0 & 6,7 & 3,3 \\
\hline Neurologische Erkrankungen & 23,3 & 23,3 & 23,3 \\
\hline Epilepsie & 10,0 & 10,0 & 10,0 \\
\hline Polyneuropathie & 10,0 & 6,7 & 13,3 \\
\hline Chronisch entzündliche ZNS-Erkrankungen & 3,3 & 6,7 & 0 \\
\hline Erkrankungen des Herz-Kreislauf-Systems & 33,3 & 40,0 & 26,7 \\
\hline KHK & 10,0 & 20,0 & 0 \\
\hline Myokardinfarkt & 3,3 & 3,3 & 3,3 \\
\hline Rhythmusstörungen & 25,0 & 26,7 & 23,3 \\
\hline Arterieller Hypertonus & 53,3 & 53,3 & 53,3 \\
\hline Stoffwechselerkrankungen & 25,0 & 26,7 & 23,3 \\
\hline Schilddrüsenerkrankungen & 23,3 & 23,3 & 23,3 \\
\hline Diabetes mellitus & 3,3 & 3,3 & 3,3 \\
\hline
\end{tabular}




\subsubsection{Leberwerte und abdomensonographische Befunde}

\subsection{Leberwerte}

Durch Aktenrecherche erfassten wir Laborwerte wie Leberwerte und Harnsäure, welche häufig bei einer Alkohol- und Medikamentenabhängigkeit pathologisch verändert sind. Weiterhin erfolgte ein Vergleich der Werte des gesamten Kollektivs und spezifisch der Patientengruppe, die im Vorfeld Lorazepam eingenommen hatte.

Zur Beurteilung der Leberzellschädigung dokumentierten wir die Höhe der Leberenzyme. Als empfindlichsten Indikator für Störungen der Integrität der Leberzellen und alkoholtoxische Schädigungen ist dies die Gamma-Glutamyl-Transferase (gamma-GT). Weiterhin wurden die Glutamat-Oxalazetat-Transaminase (GOT) und die Glutamat-Pyruvat-Transaminase (GPT) erfasst. Zusätzlich berechneten wir den De-Ritis-Quotienten als Quotienten aus GOT/ GPT, der mit dem Umfang der Leberzellschädigung korreliert. Auch wenn die Leberwerte ansonsten im Referenzbereich liegen, kann ein erhöhter De-Ritis-Quotient auf eine der genannten Schädigungen hinweisen Bei leichten Leberschäden liegt der De-Ritis-Quotient $<1$ bei schweren Leberzellschäden verschiebt er sich zu Gunsten der GOT und liegt bei Werten $>1$ (HEROLD 2008). Im gesamten Patientenkollektiv zeigten sich erhöhte Leberwerte (vgl. Tabelle 7). Eine Erhöhung der Leberenzyme zeigte sich in beiden Gruppen, mit deutlich schlechteren Werten in Gruppe B. Die erhöhten Werte aller drei Enzyme in Gruppe B weisen deutlich auf die alkoholtoxisch verursachten Schäden hin. Die gamma-GT lag in dieser Gruppe im Mittel bei 95,7 U/l (Referenzbereich bis 60 U/l), wobei die Medianwerte hier für wenige Extremwerte sprechen, die möglicherweise zu einem erhöhten Mittelwert führten. Die GOT lag bei 46,4 U/l (Referenzbereich bis $38 \mathrm{U} / \mathrm{l}$ ) und die GPT bei 41,37 U/l (Referenzbereich bis 41 U/l). Am deutlichsten zeigt sich das Ausmaß der Schädigung im DeRitis-Quotienten, der mit einem Mittelwert von 1,24 bereits auf eine schwere Leberschädigung hinweist. Es zeigten sich allerdings auch in der isoliert benzodiazepinabhängigen Gruppe A erhöhte Werte von gamma-GT und GOT. Der De-Ritis-Quotient lag bei 1,04 was auf eine dezente mögliche lebertoxische Wirkung der Benzodiazepine hinweist. In Gruppe A zeigten sich etwas stärkere Erhöhungen von Gamma-GT, GOT und GPT der Teilnehmer, die im Vorfeld Lorazepam eingenommen hatten, in Gruppe B traf dies für die GOT und GPT ebenfalls zu. Deutliche Gruppenunterschiede ergaben sich jedoch nicht. Der De-RitisQuotient lag in der Gruppe der Teilnehmer mit vorausgegangener Lorazepammedikation etwas niedriger. Signifikante Gruppenunterschiede ergaben sich im Chi-Quadrat-Test aber 
auch hier nicht ( $p=0,527 ;$ n.s.). Aufgrund der niedrigen Teilnehmerzahl mit vorausgegangener Lorazepammedikation ( $\mathrm{n}=10)$ sind diese Ergebnisse nur sehr eingeschränkt beurteilbar.

Tabelle 7: Leberwerte, De-Ritis-Quotient und Harnsäure des gesamten Kollektivs (n=60), in der Gruppe A ( $(n=30)$ und gesondert für die Teilnehmer der Gruppe A, die Lorazepam einnahmen (n=4), in Gruppe B $(n=30)$ und gesondert für die Teilnehmer in Gruppe B, die Lorazepam einnahmen (n=6). Angabe in Mittelwert, Median und Standardabweichung (SD).

\begin{tabular}{|c|c|c|c|c|c|}
\hline & $\begin{array}{l}\text { Gesamte } \\
\text { Studiengrupe }\end{array}$ & $\begin{array}{l}\text { Gruppe A } \\
\text { Teilnehmer } \\
\text { alle Benzos }\end{array}$ & $\begin{array}{l}\text { Gruppe A } \\
\text { Teilnehmer } \\
\text { mit } \\
\text { Lorazepam } \\
\text { Medikation } \\
(n=4)\end{array}$ & \begin{tabular}{|l|} 
Gruppe B \\
Teilnehmer \\
alle Benzos
\end{tabular} & $\begin{array}{l}\text { Gruppe B } \\
\text { Teilnehmer } \\
\text { mit } \\
\text { Lorazepam } \\
\text { Medikation } \\
(\mathrm{n}=6)\end{array}$ \\
\hline \multicolumn{6}{|c|}{ Laborwerte (Referenzb. der UMG) } \\
\hline \multicolumn{6}{|c|}{ Gamma-GT (Referenzb.: Frauen bis $40 \mathrm{U} / \mathrm{l}$, Männer bis $60 \mathrm{U} / \mathrm{l})$} \\
\hline Mittelwert & 80,93 & 66,13 & 85,5 & 95,73 & 49,8 \\
\hline Median & 48 & 54 & 49 & 38 & 29 \\
\hline SD & 113,24 & 62,45 & 84,5 & 147,43 & 46,0 \\
\hline \multicolumn{6}{|c|}{ GOT (Referenzb.: Frauen bis $32 \mathrm{U} / \mathrm{l}$, Männer bis $38 \mathrm{U} / \mathrm{l}$ ) } \\
\hline Mittelwert & 39,1 & 31,77 & 40,5 & 46,37 & 55,8 \\
\hline Median & 25 & 25 & 27 & 25 & 24,5 \\
\hline SD & 42,69 & 26,83 & 32,0 & 53,65 & 64,3 \\
\hline \multicolumn{6}{|c|}{ GPT (Referenzb.: Frauen bis 31 U/l, Männer bis $41 \mathrm{U} / \mathrm{l}$ ) } \\
\hline Mittelwert & 37,86 & 34,37 & 48,0 & 41,37 & 66 \\
\hline Median & 22,5 & 23,5 & 29 & 22 & 27,5 \\
\hline SD & 38,42 & 28,61 & 46,5 & 46,47 & 72,5 \\
\hline \multicolumn{6}{|c|}{ De-Ritis-Quotient (Refernzb. 0,6- 0,8) Leichte Schädigung < 1 Schwere Schädigung >1 } \\
\hline Mittelwert & 1,14 & 1,04 & 0,91 & 1,24 & 0,97 \\
\hline Median & 1,02 & 0,95 & 0,93 & 1,08 & 0,92 \\
\hline SD & 0,45 & 0,41 & 0,13 & 0,47 & 0,41 \\
\hline \multicolumn{6}{|c|}{ Harnsäure (Referenzb. < 6,4 mg/ dl) } \\
\hline Mittelwert & 5,81 & 5,53 & 5,2 & 6,08 & 6,2 \\
\hline Median & 5,3 & 5,3 & 4,5 & 5,9 & 6,0 \\
\hline SD & 2,34 & 2,08 & 1,9 & 2,59 & 1,5 \\
\hline
\end{tabular}

\subsection{Abdomensonographische Befunde}

Ebenfalls durch Aktenrecherche erfassten wir alle vorhandenen Abdomen-Sonographien $(n=55)$. Ziel war die Auswertung insbesondere pathologischer Veränderungen der Leber die häufig bei alkoholabhängigen Patienten auftreten, aber auch im Rahmen von Benzodiazepinabhängigkeit vorkommen. Wir dokumentierten Normbefunde, bildgebende Veränderungen wie Steatosis hepatis, Hepatomegalie und Splenomegalie und klinisch relevante Veränderungen wie Aszites und Leberzirrhose (vgl. Tabelle 8). In der gesamten Studiengruppe zeigte sich mit 41,8\% ein hoher Anteil an abdomensonographisch erhobenen Veränderungen. In Gruppe A zeigten sich diese Veränderungen bei 21,4\% der Patienten, während der Anteil in Gruppe B bei 63\% lag. Die Gruppen unterschieden sich somit deutlich voneinander, was auf 
die in Gruppe B zusätzlich vorhandenen alkoholtoxischen Effekte zurückzuführen ist. Klinisch relevante Veränderungen im Sinne von Aszites oder Leberzirrhose wiesen nur Patienten mit einer Alkohol- und Benzodiazepinabhängigkeit auf. Der Anteil lag dabei in Gruppe B mit nur 3,7\% erstaunlich niedrig.

Tabelle 8: Sono-Abdomen-Befunde des gesamten Kollektivs $(n=60)$, in Gruppe A $(n=30)$ und in Gruppe B $(n=30)$. Angabe in Prozent und Anzahl.

\begin{tabular}{|c|c|c|c|c|c|c|}
\hline & \multicolumn{2}{|c|}{$\begin{array}{c}\text { Gesamte Gruppe } \\
(n=55)\end{array}$} & \multicolumn{2}{|c|}{$\begin{array}{c}\text { Gruppe A } \\
(n=28)\end{array}$} & \multicolumn{2}{|c|}{$\begin{array}{c}\text { Gruppe B } \\
(\mathrm{n}=27)\end{array}$} \\
\hline & Prozent & Anzahl & Prozent & Anzahl & Prozent & Anzahl \\
\hline \multicolumn{7}{|c|}{ Sono-Abdomen-Befunde } \\
\hline Normbefund & 56,4 & 31 & 78,6 & 22 & 33,3 & 9 \\
\hline \multicolumn{7}{|c|}{ Bildgebende Veränderungen Steatosis hepatis, Splenomegalie, Hepatomegalie } \\
\hline & 41,8 & 23 & 21,4 & 6 & 63,0 & 17 \\
\hline \multicolumn{7}{|c|}{ eränderung Aszites, Zirrhose } \\
\hline & 1,8 & 1 & 0 & 0 & 3,7 & \\
\hline
\end{tabular}

\subsubsection{Bildgebende pathologische Befunde des Neurokraniums}

Bildgebende pathologische Befunde des Neurokraniums erfassten wir durch die Auswertung von CT- und MRT-Befunden. Einschränkend muss angemerkt werden, dass nur in ca. zwei Drittel der Fälle bildgebende Befunde vorlagen $(n=42)$. Ingesamt zeigte sich ein hohes Ausmaß an pathologischen Befunden im cCT/ cMRT (vgl. Tabelle 9).

Tabelle 9: Pathologische Befunde des Neurokraniums des gesamten Kollektivs (n=42), in Gruppe A $(n=19)$ und in Gruppe B (n=23). Angabe in Prozent und Anzahl.

\begin{tabular}{|c|c|c|c|c|c|c|}
\hline & \multicolumn{2}{|c|}{$\begin{array}{l}\text { Gesamte Studiengruppe } \\
\qquad(\mathrm{n}=42)\end{array}$} & \multicolumn{2}{|c|}{$\begin{array}{c}\text { Gruppe A } \\
(n=19)\end{array}$} & \multicolumn{2}{|c|}{$\begin{array}{c}\text { Gruppe B } \\
(n=23)\end{array}$} \\
\hline & Prozent & Anzahl & Prozent & Anzahl & Prozent & Anzahl \\
\hline \multicolumn{7}{|l|}{ MRT-I CT-Befunde } \\
\hline Hirnatrophie & 35,7 & 15 & 26,3 & 5 & 43,5 & 10 \\
\hline Marklagerläsionen & 19 & 8 & 31,6 & 6 & 8,7 & 2 \\
\hline Mikroangiopathie & 11,9 & 5 & 26,3 & 5 & 0 & 0 \\
\hline Makroangiopathie & 4,8 & 2 & 5,3 & 1 & 4,3 & 1 \\
\hline
\end{tabular}

Den größten Anteil machten hirnatrophische Veränderungen aus, die im gesamten Kollektiv bei 35,7\% lagen. Im Gruppenvergleich zeigte sich mit 43,5\% ein höherer Anteil in Gruppe B. Statistisch signifikante Unterschiede zwischen den Gruppen bestanden jedoch nicht. Einen weiteren großen Anteil machten Marklagerläsionen und mikroangiopathische Veränderungen aus, wobei sich hier ein deutlich höherer Anteil in Gruppe A zeigte. 
Makroangiopathische Veränderungen kamen in 4,8\% der Fälle vor, mit gleicher Verteilung in den Gruppen. Signifikante positive Korrelationen zwischen pathologischen Befunden des Neurokraniums, insbesondere hirnathrophischen Veränderungen, und kognitiven Defiziten im Uhrentest oder MMST, bestanden nicht (vgl. Tabelle 10).

Tabelle 10: Korrelationen (r) mit Wahrscheinlichkeit (p) im Vergleich hirnathrophischer Veränderungen mit den Ergebnissen des MMST und Uhrentest im gesamten Patientenkollektiv (n=15).

\begin{tabular}{|l|r|r|r|r|}
\hline & \multicolumn{4}{|c|}{ Kognitive Defizite } \\
\hline & \multicolumn{2}{|c|}{ MMST } & \multicolumn{2}{c|}{ Uhrentest } \\
\hline \multicolumn{1}{|c|}{ Korrelationen } & \multicolumn{1}{c|}{$\mathbf{r}$} & \multicolumn{1}{c|}{$\mathbf{p}$} & \multicolumn{1}{c|}{$\mathbf{~}$} & \multicolumn{1}{c|}{$\mathbf{p}$} \\
\hline Hirnathrophie & 0,170 & 0,282 & 0,149 & 0,346 \\
\hline Mikroangiopathie & 0,041 & 0,797 & $-0,074$ & 0,644 \\
\hline Makroangiopathie & 0,277 & 0,760 & 0,033 & 0,861 \\
\hline Marklagerläsionen & $-0,171$ & 0,279 & 0,034 & 0,908 \\
\hline
\end{tabular}

\subsubsection{EEG-Befunde}

Zur Erfassung von Hirnleistungsstörungen werteten wir alle vorhandenen EEG-Befunde aus ( $n=55)$. Pathologische Befunde zeigten sich in 9\% der Fälle. Es zeigte sich mit 13,8\% ein höherer Anteil in Gruppe A ( $\mathrm{n}=29)$.

\subsection{Kognitive Defizite benzodiazepinabhängiger Patienten}

\subsubsection{Kognitive Defizite im Uhrentest (CDT)}

Zur orientierenden Erfassung von Hirnleistungsstörungen verwendeten wir den Uhrentest/ Clockdrawing Test (CDT nach SHULMAN et al. 1993), der insbesondere die Beurteilung von visuokonstruktiven Fähigkeiten und Handlungsplanung erlaubt. Im Uhrentest wurden Scores von 1 bis 6 vergeben wobei ein Score von über drei oder gleich drei als pathologisch anzusehen ist.

Die folgende Graphik 4 zeigt die Testergebnisse im gesamten Kollektiv und in beiden Gruppen. Eine Teilnehmerin war aufgrund eines starken Tremors nicht in der Lage den Test durchzuführen. Bei Betrachtung des gesamten Kollektivs zeigte sich, dass nur etwa ein Viertel der Patienten einen Score von 1, entsprechend einer korrekten Darstellung der Uhr, erreichte. Mehr als ein Drittel der Patienten erreichte einen pathologischen Score von 3, entsprechend einer fehlerhaften eingezeichneten Uhrzeit. Mehr als 10\% der Patienten lagen im Bereich mittelgradiger visuell-räumlicher Desorganisation und über $5 \%$ im Bereich 
schwergradiger visueller Desorganisation. Im gesamten Kollektiv zeigten sich somit insgesamt hochgradige kognitive Defizite im Uhrentest.

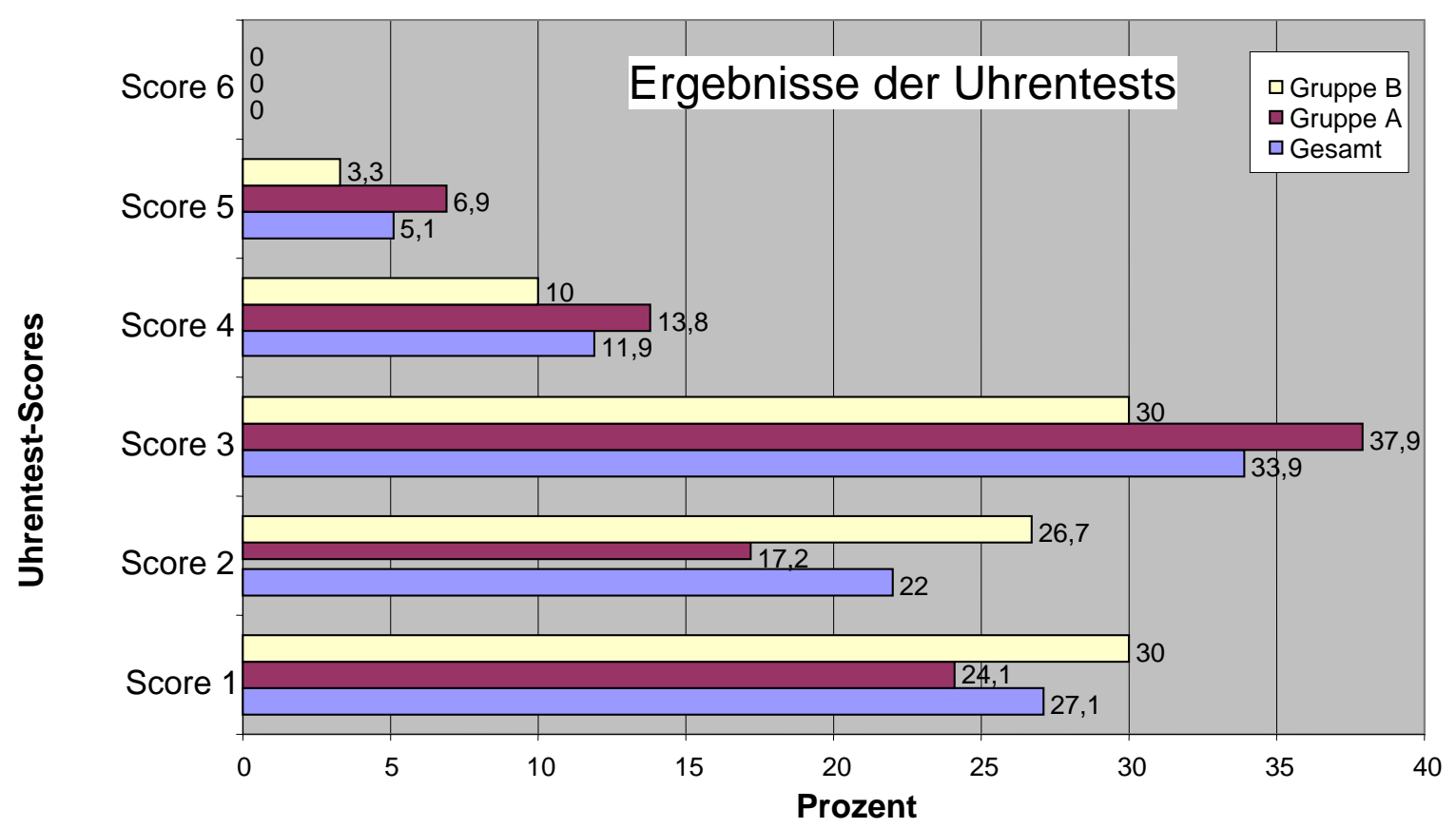

Graphik 4: Uhrentestergebnisse des gesamten Patientenkollektivs $(n=59)$ und der einzelnen Patientengruppen. Gruppe A (n=29), Gruppe B (n=30).

Die gesamte Studiengruppe $(n=59)$ erreichte im Mittel einen Score von 2,46 (SD 1,16; Median 3). Der Score lag dabei in Gruppe A ( $n=29)$ im Mittel bei 2,62 (SD 1,2; Median 3) und in Gruppe B (n=30) im Mittel bei 2,3 (SD 1,12; Median 2) (vgl. Graphik 5).

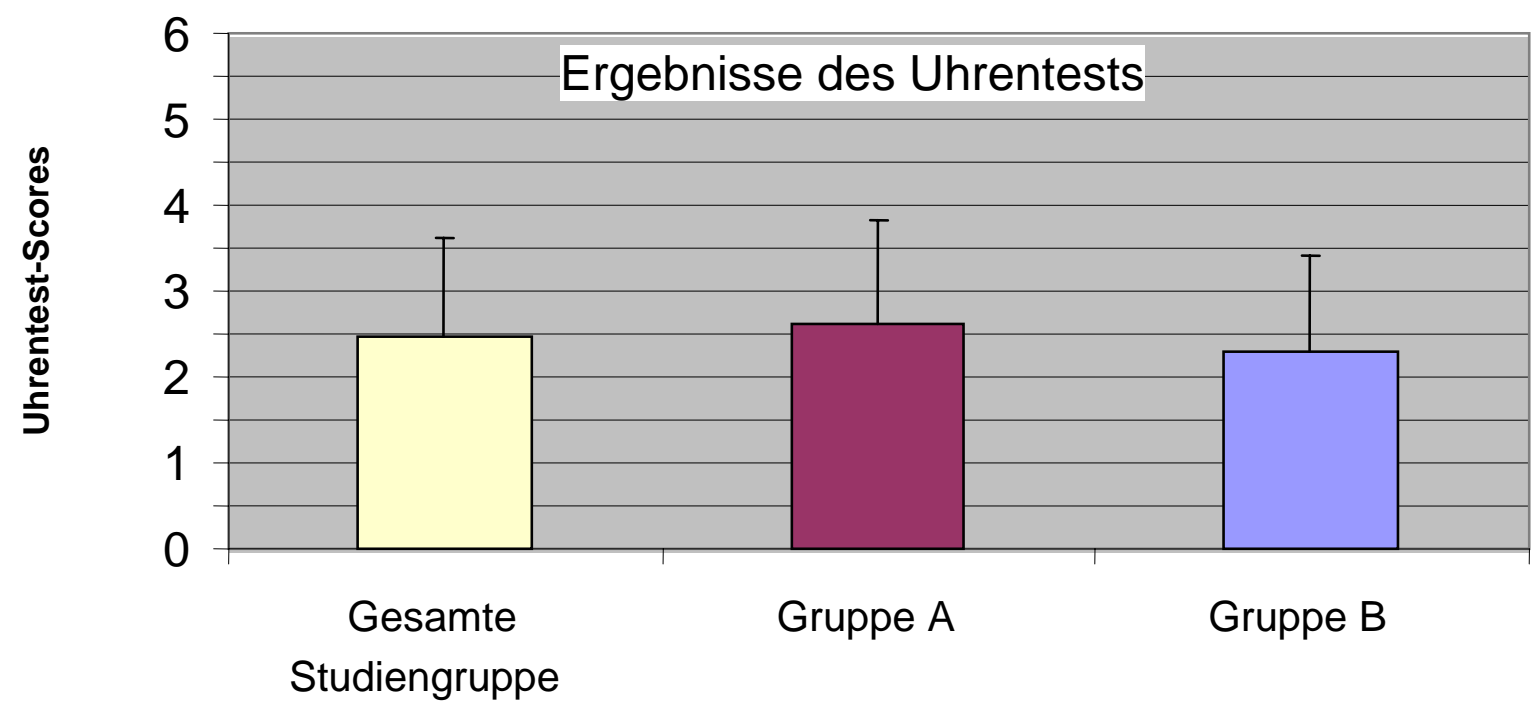

Graphik 5: Vergleich der Ergebnisse des Uhrentests. Angaben in Mittelwerten mit SD des gesamten Kollektivs (n=59) und der einzelnen Patientengruppen. Gruppe A (n=29), Gruppe B (n=30). 


\subsubsection{Mini Mental Status Test (MMST)}

Als weiterer Screeningtest zur orientierenden Erfassung kognitiver Defizite wurde der Mini Mental Status Test (MMST nach FOLSTEIN et al. 1975) durchgeführt. Die Patienten konnten hierbei maximal 30 Punkte erreichen. Von Hirnleistungsstörungen wird ab Werten von unter 25 Punkten ausgegangen. Im gesamten Kollektiv wurden im Mittel 28,3 Punkte erreicht (SD 1,6; Median 29; Spannweite 24-30). In Gruppe A lag der Mittelwert bei 28,13 Punkten (SD 1,65; Median 29) und in Gruppe B bei 28,47 Punkten (SD 1,54; Median 29). Die im Uhrentest aufgefallenen kognitiven Defizite ließen sich somit hier nicht nachvollziehen.

\subsection{Schweregrade der Abhängigkeit im EuropASI}

\subsubsection{ASI-Scores}

Um den Schweregrad der Benzodiazepin- und Alkoholabhängigkeit in verschiedenen potentiellen Problembereichen zu erfassen, wählten wir den EuropASI (nach GSELLHOFER et al. 1999) als standardisierten Fragebogen. Es wurden Composite-Scores für die Problembereiche körperlicher Zustand, Arbeits- und Unterhaltssituation, Alkoholgebrauch, Drogengebrauch, rechtliche Situation, Familie und Sozialbeziehungen sowie psychischer Status errechnet. Für jeden einzelnen Bereich konnten Punktwerte zwischen 0 und 1 erreicht werden. Hierbei steht ein Punktwert von 0-0,44 für eine geringe, Punktwerte von 0,45-0,74 für eine mäßige und Werte von 0,75-1 für eine starke Ausprägung eines Problems. Diese CompositScores basieren nur auf der Situation des Patienten innerhalb der letzten 30 Tage, somit sind sie für eine Erfassung der Veränderung der aktuellen Situation geeignet. Wir befragten die Patienten über die letzten 30 Tage vor dem Entzug, um eine reale Situationsabschätzung vornehmen zu können. Einen Überblick über die einzelnen Composite-Scores des EuropASI berechnet für das gesamte Patientenkollektiv zeigt die folgende Tabelle 11. Es zeigte sich im Mittel im gesamten Kollektiv eine Ausprägung von Problemen in den Bereichen körperlicher Zustand, ökonomischer Status und psychische Situation. Wobei sich eine höhere Ausprägung der körperlichen Probleme in Gruppe A zeigte. Es interessierten insbesondere die Parameter des Drogengebrauchs, um die Problematik der Benzodiazepin- Einnahme verifizieren zu können. Wie in der Tabelle 11 veranschaulicht, lagen die Scores, die wir zur Abschätzung des Schweregrades der Abhängigkeit verwendeten, definiert als Drogengebrauch 1 und Drogengebrauch 2, im Mittel bei 0,23 bzw. 0,25 betreffend das gesamten Kollektivs (SD 0,06 bzw SD 0,07 Spanne 0,11-0,35 bzw. 0,09-0,39). 
Tabelle 11: Abbildung der ASI-Scores der verschiedenen Problembereiche im gesamten Kollektiv ( $n=60)$, in Gruppe A $(n=30)$ und in Gruppe B $(n=30)$ angegeben in Mittelwert, Median und SD.

\begin{tabular}{|l|r|r|r|r|r|r|r|r|r|}
\hline ASI-Scores & \multicolumn{3}{|c|}{ Ges. Studiengruppe $(\mathbf{n}=\mathbf{6 0})$} & \multicolumn{3}{|c|}{ Gruppe A (n=30) } & \multicolumn{3}{|c|}{ Gruppe B (n=30) } \\
\hline & Mittelwert & Median & \multicolumn{1}{c|}{ SD } & Mittelwert & Median & SD & Mittelwert & Median & SD \\
\hline Körperlicher Zustand & $\mathbf{0 , 4 9}$ & 0,49 & 0,34 & $\mathbf{0 , 5 6}$ & 0,64 & 0,34 & $\mathbf{0 , 4 2}$ & 0,43 & 0,34 \\
\hline Ökonomischer Status & $\mathbf{0 , 6 5}$ & 0,67 & 0,35 & $\mathbf{0 , 6 8}$ & 0,67 & 0,34 & $\mathbf{0 , 6 1}$ & 0,67 & 0,37 \\
\hline Arbeitssituation & $\mathbf{0 , 2 8}$ & 0,17 & 0,32 & $\mathbf{0 , 3 0}$ & 0,17 & 0,17 & $\mathbf{0 , 2 6}$ & 0,17 & 0,30 \\
\hline Alkoholgebrauch & $\mathbf{0 , 2 8}$ & 0,02 & 0,33 & $\mathbf{0 , 0 0}$ & 0,00 & 0,01 & $\mathbf{0 , 5 5}$ & 0,65 & 0,26 \\
\hline Drogengebrauch 1 & $\mathbf{0 , 2 3}$ & 0,21 & 0,06 & $\mathbf{0 , 2 2}$ & 0,21 & 0,05 & $\mathbf{0 , 2 4}$ & 0,25 & 0,06 \\
\hline Drogengebrauch 2 & $\mathbf{0 , 2 5}$ & 0,24 & 0,07 & $\mathbf{0 , 2 4}$ & 0,23 & 0,05 & $\mathbf{0 , 2 7}$ & 0,28 & 0,08 \\
\hline Rechtliche Situation & $\mathbf{0 , 0 1}$ & 0,00 & 0,08 & $\mathbf{0 , 0 0}$ & 0,00 & 0,02 & $\mathbf{0 , 0 3}$ & 0,00 & 0,11 \\
\hline Familiäre Situation & $\mathbf{0 , 3 2}$ & 0,33 & 0,26 & $\mathbf{0 , 2 5}$ & 0,28 & 0,23 & $\mathbf{0 , 3 9}$ & 0,40 & 0,27 \\
\hline Soziale Beziehungen & $\mathbf{0 , 0 8}$ & 0,00 & 0,14 & $\mathbf{0 , 0 6}$ & 0,00 & 0,12 & $\mathbf{0 , 1 0}$ & 0,00 & 0,16 \\
\hline Psychischer Status & $\mathbf{0 , 4 9}$ & 0,52 & 0,16 & $\mathbf{0 , 5}$ & 0,52 & 0,14 & $\mathbf{0 , 4 9}$ & 0,52 & 0,17 \\
\hline
\end{tabular}

In der Gruppe A lagen die Scores dabei im Mittel bei 0,22 bzw. 0,24 (SD 0,05 bzw. 0,05). In der Gruppe B bei 0,24 bzw. 0,27 (SD 0,06 bzw. 0,07). Die Gruppe der zusätzlich alkoholabhängigen Patienten wies somit einen etwas höheren Wert auf, bei insgesamt jedoch verwunderlich niedrigen Scores bei aus klinischer Sicht teilweise schwer benzodiazepinabhängigen Patienten. Graphik 6 zeigt die Verteilung der ASI-Scores im gesamten Patientenkollektiv und in den einzelnen Gruppen.

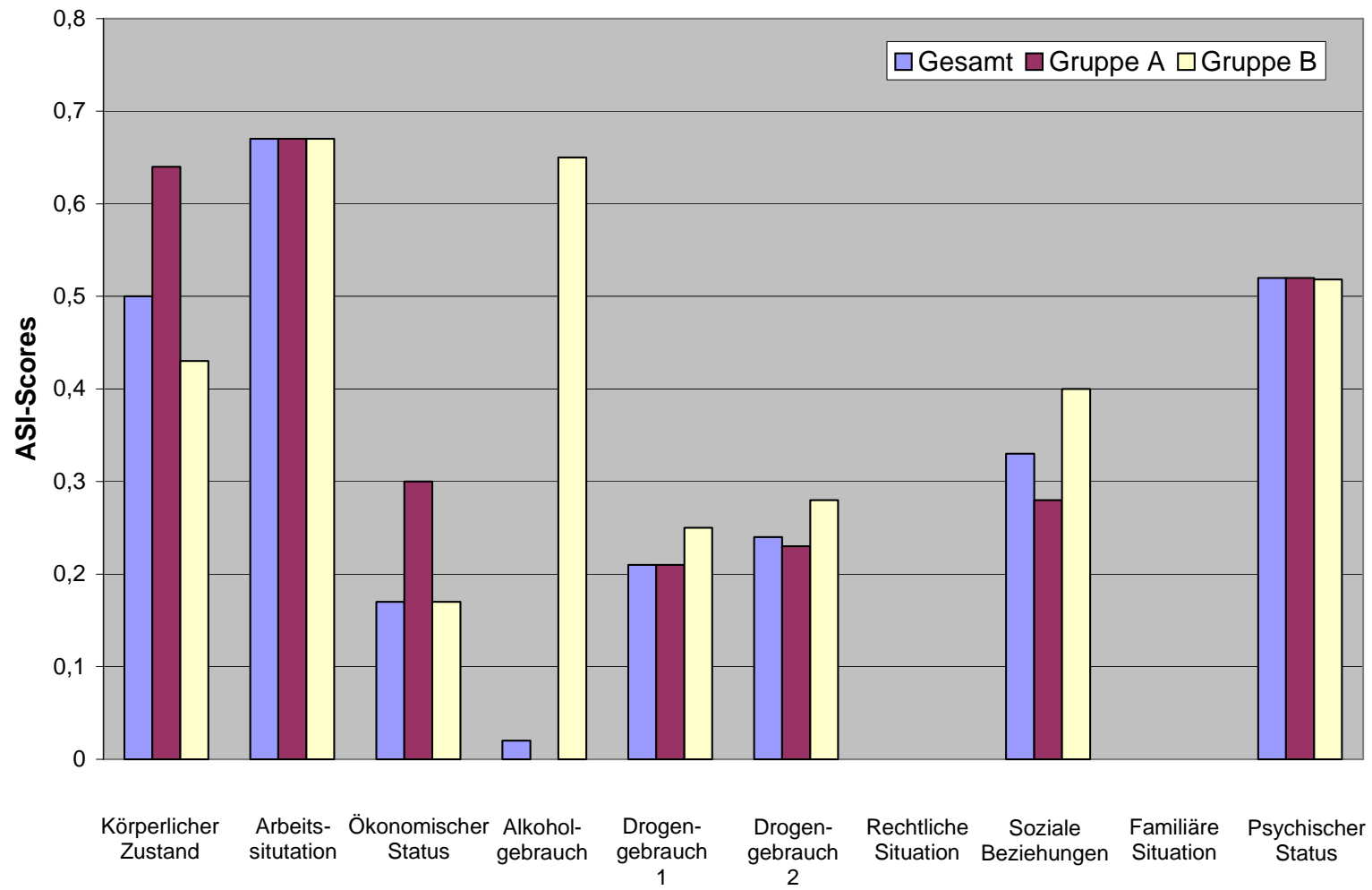

\section{Problembereich}

Graphik 6: Abbildung der ASI-Scores im gesamten Kollektiv $(n=60)$, in Gruppe A $(n=30)$ und in Gruppe $B(n=30)$ angegeben als Mittelwerte. 


\subsubsection{ASI-Schweregrade}

Neben den Scores wurden für die einzelnen Problembereiche Schweregrade definiert. In jedem der Bereiche wurde hierbei durch eine 5-Punkte-Rating-Skala (0-4) eine Selbsteinschätzung des Patienten getroffen, in der der Schweregrad der Probleme und das Ausmaß, in dem der Patient eine Behandlung als nötig erachtet, gemessen werden. Danach erfolgte die Schweregrad Einschätzung des Untersuchenden in einer 9-Punkte-Rating-Skala (0-9). Ein Wert zwischen 0-1 entspricht keinem echten Problem und sieht somit keinen Behandlungsbedarf vor. Höhere Werte zeigen ein Problem an, so dass eine Behandlung in Betracht gezogen werden sollte und Werte um 8-9 zeigen ein extremes Problem mit absoluter Behandlungsnotwendigkeit an. Tabelle 12 gibt einen Überblick der Schweregrade des EuropASI, berechnet für das gesamte Patientenkollektiv.

Tabelle 12: Abbildung der ASI-Schweregrade der verschiedenen Problembereiche im gesamten Kollektiv $(n=60)$, in Gruppe A $(n=30)$ und in Gruppe B $(n=30)$ angegeben in Mittelwert, Median und SD.

\begin{tabular}{|c|c|c|c|c|c|c|c|c|c|}
\hline \multirow[t]{2}{*}{ ASI-Schweregrad } & \multicolumn{3}{|c|}{$\begin{array}{l}\text { Gesamte Studiengruppe } \\
\qquad(n=60)\end{array}$} & \multicolumn{3}{|c|}{$\begin{array}{c}\text { Gruppe A } \\
(n=30)\end{array}$} & \multicolumn{3}{|c|}{$\begin{array}{c}\text { Gruppe B } \\
(n=30)\end{array}$} \\
\hline & Mittelwert & Median & $\mathrm{SD}$ & Mittelwert & Median & SD & Mittelwert & Median & SD \\
\hline Körperlicher Zustand & 4,58 & 5 & 2,59 & 4,93 & 6 & 2,52 & 4,23 & 4 & 2,65 \\
\hline $\begin{array}{l}\text { Ökonomischer Status/ } \\
\text { Arbeitssituation }\end{array}$ & 3,38 & 3 & 2,94 & 3,17 & 3 & 3,11 & 3,58 & 3 & 2,80 \\
\hline Alkoholgebrauch & 3,13 & 2 & 3,23 & 0,20 & 0 & 0,48 & 6,07 & 7 & 1,86 \\
\hline Drogengebrauch & 6,47 & 6 & 1,24 & 6,43 & 6 & 1,07 & 6,45 & 6 & 1,40 \\
\hline Rechtliche Situation & 0,38 & 0 & 1,29 & 0,13 & 0 & 0,73 & 0,63 & 0 & 1,65 \\
\hline $\begin{array}{l}\text { Familiäre Situation/ } \\
\text { Soziale Beziehungen }\end{array}$ & 4,37 & 5 & 2,58 & 3,77 & 4 & 2,56 & 4,97 & 5 & 2,48 \\
\hline Psychischer Status & 6,88 & 7 & 1,64 & 6,73 & 7 & 1,82 & 7,03 & 7 & 1,45 \\
\hline
\end{tabular}

Auch in der Ermittlung der Schweregrade zeigte sich eine Ausprägung von Problemen, besonders in den Bereichen körperlicher Zustand, ökonomischer Status und psychischer Status. Die Werte lagen dabei in beiden Gruppen ähnlich verteilt. Zusätzlich zeigten sich Probleme in der familiären Situation mit einer höheren Ausprägung in Gruppe B. Die Schweregrade des Drogengebrauchs lagen dabei im Mittel, wie auch in der Tabelle ersichtlich, für das gesamte Kollektiv bei 6,4 Punkten (SD 1,3; Spanne 4-9). Die Werte in Gruppe A lagen dabei im Mittel bei 6,4 Punkten (SD 1). Die Werte in Gruppe B bei 6,5 Punkten (SD 1,4). In der Bewertung der Schweregrade zeigte sich somit an den durchschnittlich hohen Werten wesentlich deutlicher das Ausmaß der Benzodiazepinabhängigkeit. 
Die folgende Graphik 7 zeigt die Verteilung der ASI-Schweregrade im gesamten Patientenkollektiv und in den einzelnen Gruppen.

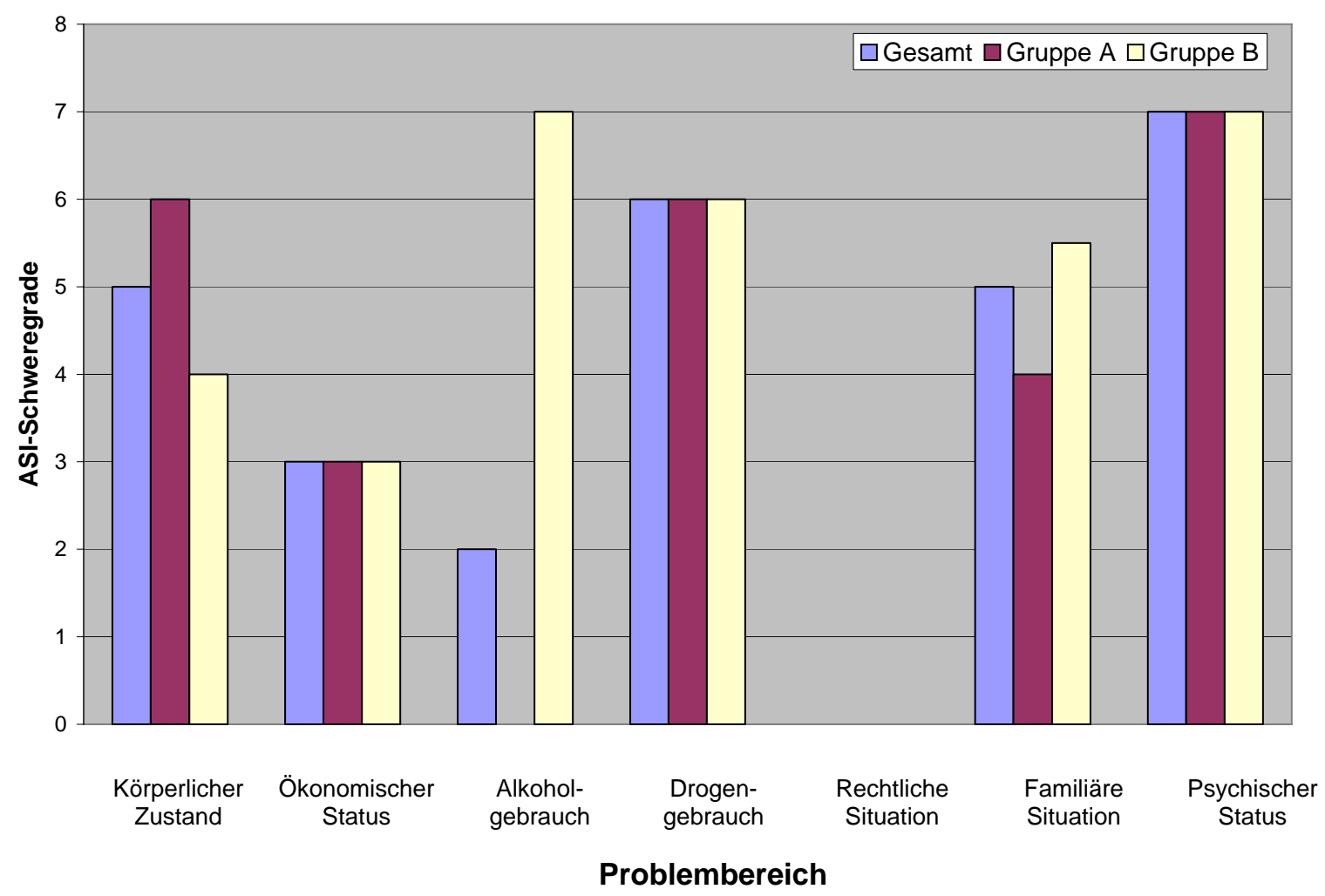

Graphik 7: Abbildung der ASI-Schweregrade im gesamten Kollektiv $(n=60)$, in Gruppe A $(n=30)$ und in Gruppe B ( $n=30)$ angegeben als Mittelwerte.

\subsection{Entzugssymptome gemessen durch die Physician Withdrawal Checklist}

Die Physician Withdrawal Checklist (PWC nach RICKELS et al. 1990) wurde verwendet, um Symptome zu erfassen, die häufig im Benzodiazepinentzug auftreten. Die Schwere der Entzugssymptome wurde von 0 = ,aktuell keine“, 1 = „leichte“, 2 = „mittelschwere“ bis 3 = „schwere“ bewertet. Die Einschätzung der Entzugssymptome nach RICKELS wurde im gesamten Kollektiv bei 32 der 60 teilnehmenden Patienten vorgenommen, da uns die Tabellen von Herrn Rickels erst nach Studienbeginn zur Verfügung gestellt werden konnten. Die folgende Tabelle 13 zeigt die Verteilung der möglichen Entzugssymptome, angegeben als Anzahl der Patienten pro Symptom und Scorewert. 
Tabelle 13: Abbildung der Rickels-Scores. Angabe in Anzahl der Patienten pro Symptom und Score. Auswertung für das gesamte Kollektiv ( $n=32)$.

\begin{tabular}{|c|c|c|c|c|}
\hline & \multicolumn{4}{|c|}{ Rickels-Scores der gesamten Studiengruppe $(n=32)$} \\
\hline & Score 0 & Score 1 & Score 2 & Score 3 \\
\hline & Anzahl & Anzahl & Anzahl & Anzahl \\
\hline \multicolumn{5}{|l|}{ Entzugssymptome } \\
\hline Angst & 6 & 6 & 7 & 13 \\
\hline Depressionen & 7 & 5 & 10 & 10 \\
\hline Schlaflosigkeit & 9 & 4 & 9 & 10 \\
\hline Ermüdung & 9 & 7 & 11 & 5 \\
\hline Ruhelosigkeit & 12 & 4 & 12 & 4 \\
\hline Zittern & 14 & 5 & 8 & 5 \\
\hline Konzentrationsschwierigkeiten & 12 & 9 & 9 & 2 \\
\hline Kopfschmerz & 18 & 4 & 4 & 6 \\
\hline Schwitzen & 15 & 8 & 5 & 4 \\
\hline Appetitverlust & 19 & 3 & 5 & 5 \\
\hline Übelkeit & 20 & 4 & 2 & 6 \\
\hline Myalgien & 18 & 7 & 5 & 2 \\
\hline Schwindel & 19 & 6 & 5 & 2 \\
\hline Schwäche & 17 & 10 & 4 & 1 \\
\hline Geräuschempfindlichkeit & 18 & 7 & 6 & 1 \\
\hline Parästhesien & 20 & 5 & 5 & 2 \\
\hline Koordinationsprobleme & 20 & 7 & 4 & 1 \\
\hline Gereiztheit & 20 & 7 & 5 & 0 \\
\hline Depersonalisation & 27 & 1 & 2 & 2 \\
\hline Durchfälle & 29 & 2 & 0 & 1 \\
\hline
\end{tabular}

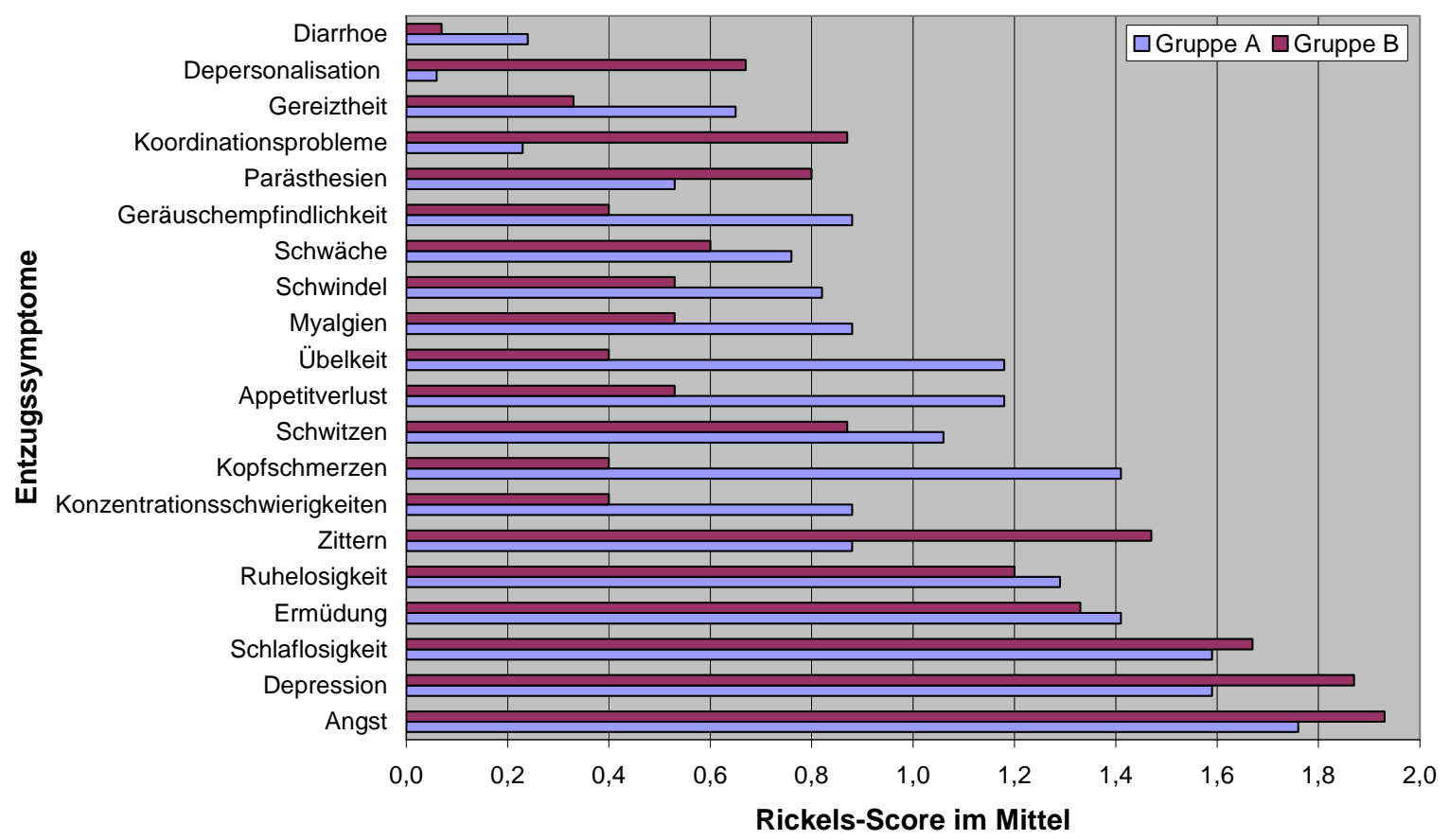

Graphik 8: Vergleich der Mittelwerte der Rickels-Scores aufgelistet für jedes Symptom. Auswertung für Gruppe A (n=17) und Gruppe B (n=15). 
Graphik 8 zeigt einen Vergleich der Mittelwerte der Rickels-Scores der einzelnen Entzugssymptome.

Im Vordergrund stehende Symptome des Benzodiazepinentzugs waren somit Angst, Depression bzw. dysphorische Stimmung und Schlaflosigkeit. Darauf folgten Symptome wie Ermüdung, Ruhelosigkeit, Zittern und Konzentrationsschwierigkeiten. Wir berechneten weiterhin die Summe aller Itemscores für jeden Patienten mit einem Maximalwert von 60 Punkten. Die mittleren Werte für das gesamte Kollektiv lagen dabei bei 18,7 Punkten (SD 8,9; Median 17; Spanne 6-38). Die Werte in Gruppe A ( $n=17)$ lagen im Mittel bei 19,5 Punkten (SD 8,8; Median 18). Die Werte in Gruppe B $(n=15)$ lagen im Mittel bei 17,5 Punkten (SD 9,4; Median 17,5). Die Gruppen unterschieden sich damit kaum in der Ausprägung der Entzugssymptome.

\subsection{Hypothesen}

Im weiteren Abschnitt werden Vergleiche der Gruppe A als isoliert benzodiazepinabhängige Patienten und Gruppe B als benzodiazepin- und alkoholabhängige Patienten dargestellt. Zur Überprüfung statistisch signifikanter Unterschiede wurde bei ordinal- und intervallskalierten Daten auf den Mann-Whitney-U-Test zurückgegriffen. Dichotome Daten wurden mit Hilfe des Chi-Quadrat-Tests ausgewertet.

\subsubsection{Hypothese 1: Benzodiazepinabhängige Patienten unterscheiden sich von benzodiazepin- und alkoholabhängigen Patienten im Benzodiazepinentzug hinsichtlich psychiatrischer und somatischer Komorbiditäten}

Zur Überprüfung signifikanter Unterschiede in der Häufigkeit des Auftretens komorbider Störungsbilder wurden alle psychiatrischen und somatischen Begleiterkrankungen zwischen den beiden Gruppen verglichen.

\subsubsection{Vergleich komorbider psychiatrischer Störungsbilder}

Als Parameter komorbider psychiatrischer Störungsbilder verglichen wir die Häufigkeit des Auftretens von Angststörungen, posttraumatischen Belastungsstörungen, affektiven Störungen, Essstörungen, Psychosen und somatoformen Störungen zwischen den Gruppen. Die folgende Graphik 9 zeigt die Verteilung in den einzelnen Gruppen angegeben in Anzahl der Patienten. 


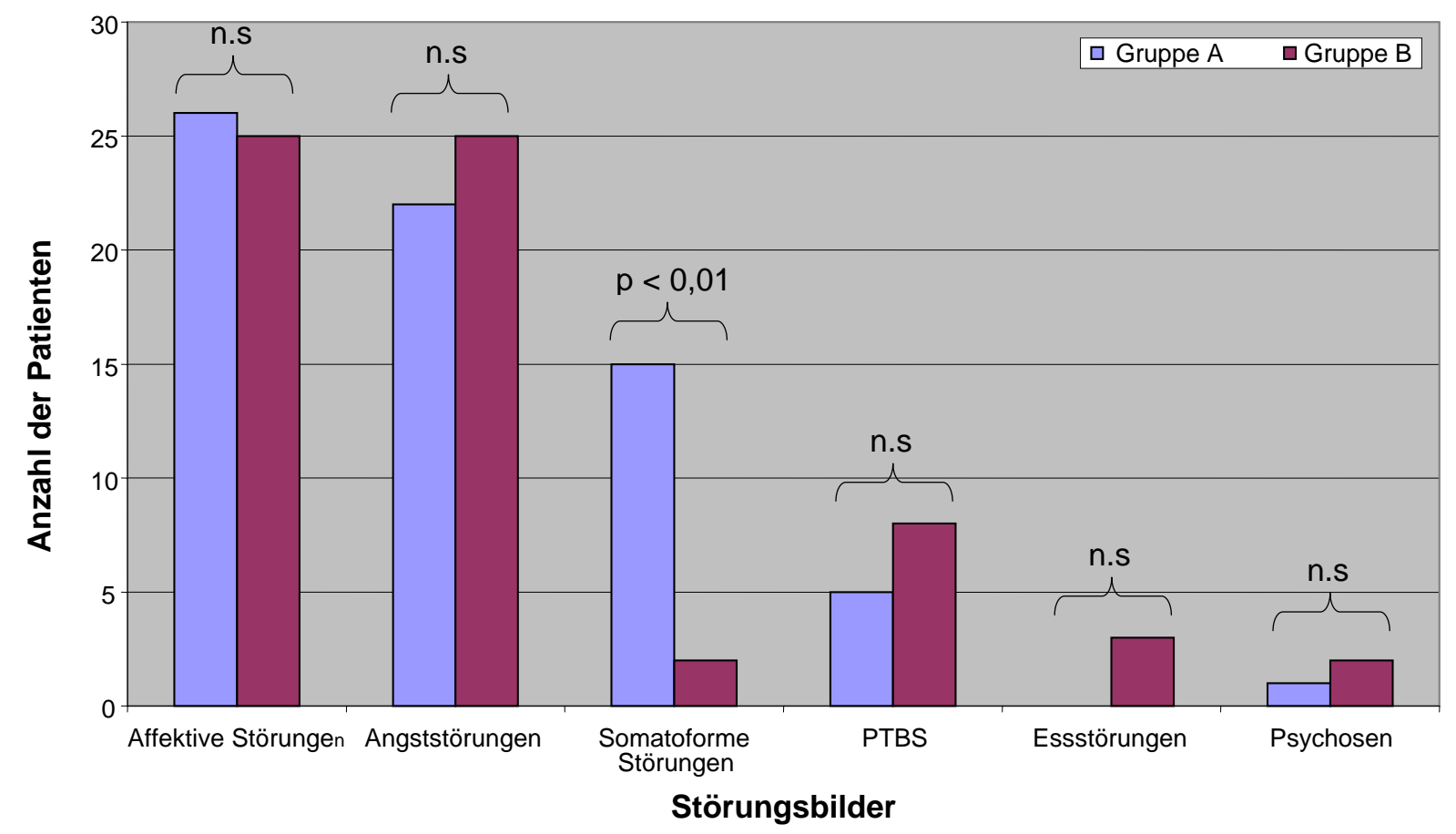

Graphik 9: Psychische komorbide Störungsbilder. Angabe in Anzahl der Patienten in Gruppe A (n=30) und Gruppe B (n=30).

Im Chi-Quadrat-Test zeigten sich hierbei keine signifikanten Unterschiede in der Häufigkeit des Auftretens von Angststörungen ( $\mathrm{p}=0,347$; n.s.), posttraumatischen Belastungsstörungen ( $\mathrm{p}=0,347$; n.s.), affektiven Störungen ( $\mathrm{p}=0,718$; n.s.), Essstörungen ( $\mathrm{p}=0,076$; n.s.), und Psychosen ( $\mathrm{p}=0,554 ;$ n.s.). Wobei sich die Gruppen hochsignifikant häufig $(\mathrm{p}<0,01)$ im Auftreten von somatoformen Störungen unterschieden, die sich in einem deutlich höheren Anteil in Gruppe A zeigten. Somit zeigte sich Hypothese 1 in Bezug auf das Auftreten psychiatrischer komorbider Störungsbilder bestätigt. Die rein benzodiazepinabhängigen Patienten zeigten signifikant mehr somatoforme Störungen.

\subsubsection{Vergleich komorbider somatischer Störungsbilder}

Als Parameter komorbider somatischer Störungsbilder verglichen wir die Häufigkeit des Auftretens von Begleiterkrankungen, Leberwerte und abdomensonographische Befunde, bildgebende pathologische Befunde des Neurokraniums, EEG-Befunde und Entzugskrampfanfälle.

\subsection{Begleiterkrankungen}

Es erfolgte der Vergleich komorbider somatischer Begleiterkrankungen wie Störungen des Bewegungsapparates, chronischen Schmerzsyndromen, neurologischen Erkrankungen, Herz- 
Kreislauf-Erkrankungen, Arteriellem Hypertonus und Stoffwechselstörungen zwischen den Gruppen. Graphik 10 zeigt die Verteilung in den einzelnen Gruppen angegeben in Anzahl der Patienten.

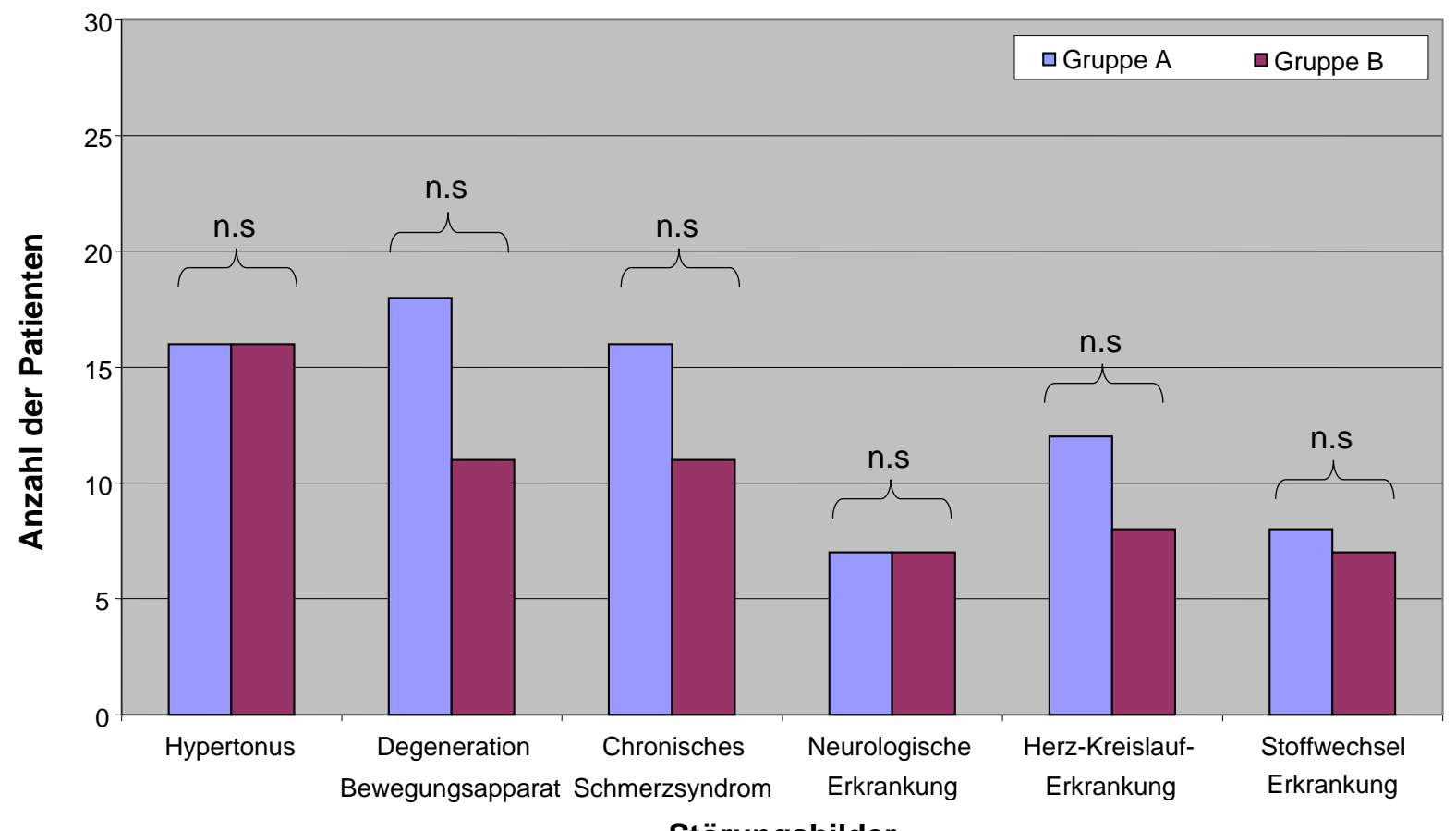

Störungsbilder

Graphik 10: Somatische komorbide Störungsbilder. Angabe in Anzahl der Patienten in Gruppe A (n=30) und Gruppe B (n=30).

Im Chi-Quadrat-Test zeigten sich hierbei trotz nomineller Unterschiede keine signifikanten Unterschiede in der Häufigkeit des Auftretens von chronischen Schmerzsyndromen ( $p=0,194$; n.s.), neurologischen Erkrankungen ( $p=0,76$; n.s.), Herz-Kreislauf-Erkrankungen ( $p=1,0$; n.s.), Arteriellem Hypertonus ( $p=0,273$; n.s.) und Stoffwechselerkrankungen ( $p=0,766$; n.s.). Im Vergleich degenerativer Störungen des Bewegungsapparates zeigte sich ein statistischer Trend ( $p=0,071 ; n . s$.$) zu vermehrtem Auftreten in Gruppe A.$

\subsection{Leberwerte}

Weiterhin erfolgte der Vergleich von Laborwerten wie Leberwerten, De-Ritis-Quotient und Harnsäure. Graphik 11 zeigt die Höhe der Transaminasen gamma-GT, GOT und GPT in den einzelnen Gruppen. Im Vergleich der Häufigkeit pathologisch erhöhter Leberwerte mittels des Mann-Whitney-U-Test ergaben sich hochsignifikante Unterschiede im Grad der Erhöhung der Gamma-GT $(\mathrm{U}=239,5 ; \mathrm{p}<0,01)$ und der GOT $(\mathrm{U}=300 ; \mathrm{p}<0,01)$. Beide lagen in der Gruppe der zusätzlich alkoholabhängigen Patienten deutlich höher, während sich keine signifikanten Unterschiede der Erhöhung der GPT (U=370; p=0,2; n.s.) zeigten. Im Vergleich der Höhe der 
De-Ritis-Quotienten und Harnsäurewerte beider Gruppen zeigten sich keine signifikanten Unterschiede ( $U=325 ; p=0,09$; n.s.).

\section{Leberwerte im Gruppenvergleich}

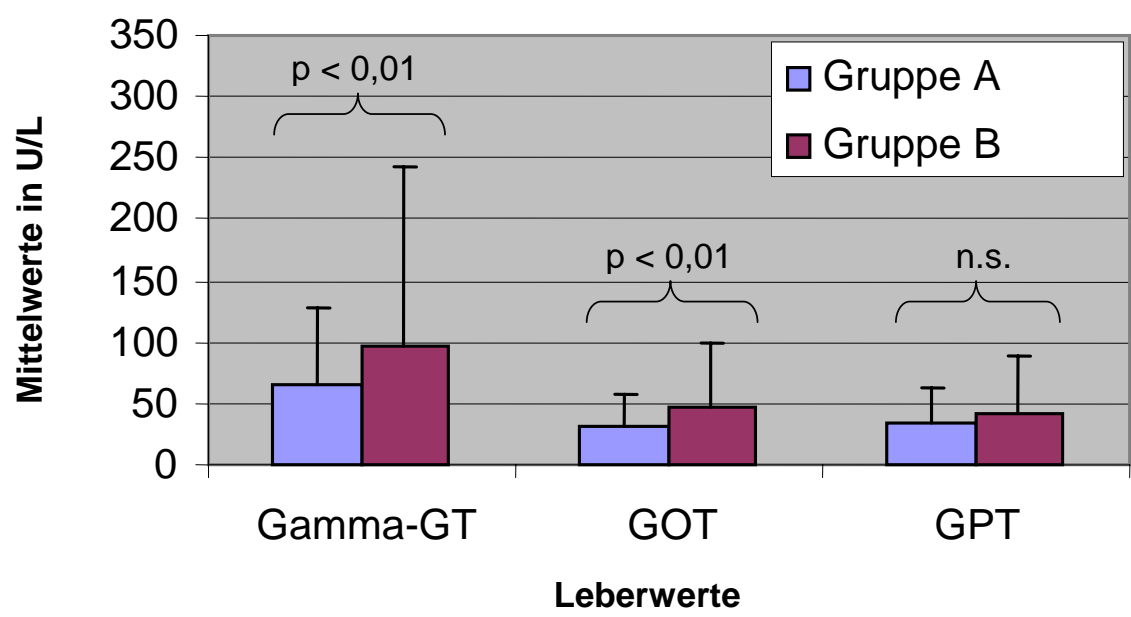

Graphik 11: Leberwerte im Vergleich zwischen den Gruppen. Gruppe A (n=30) und Gruppe B ( $n=30)$, Angabe der Mittelwerte in $U / l$.

Graphik 12 zeigt die Höhe der Harnsäurewerte, bzw. Graphik 13 die des De-Ritis-Quotienten in den einzelnen Gruppen

\section{Harnsäurewerte im Gruppenvergleich}

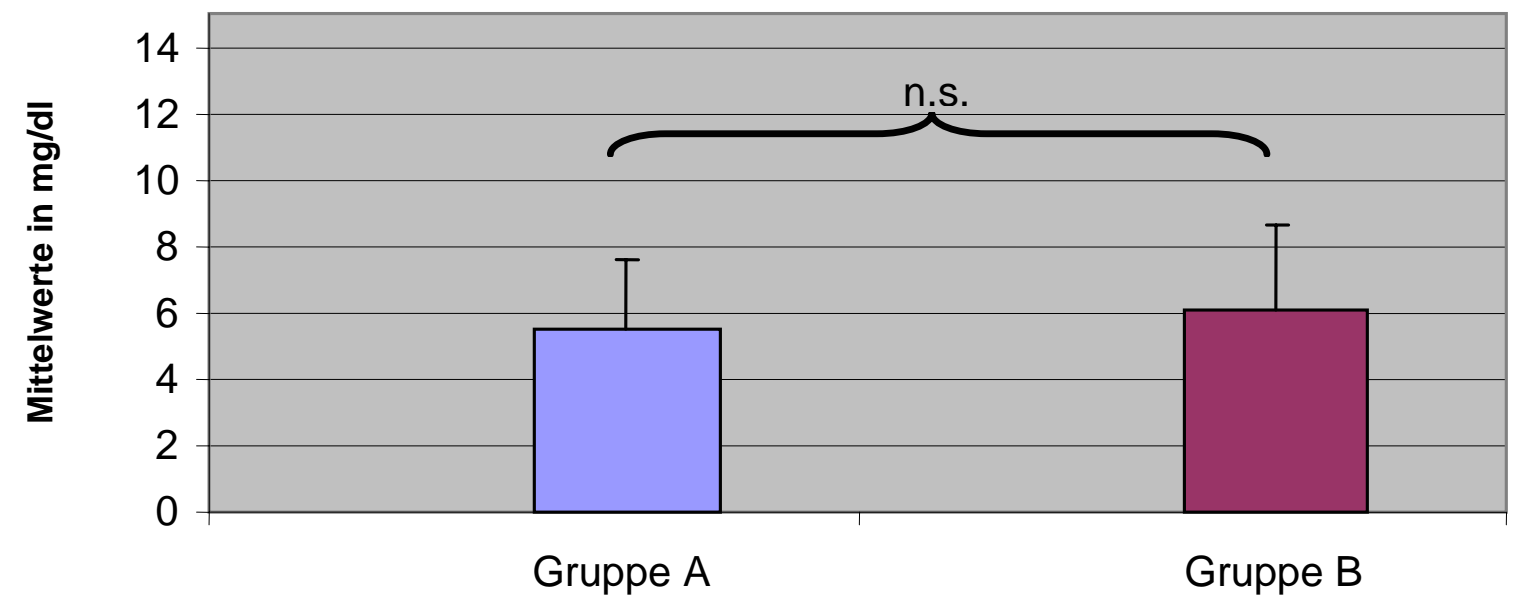

Graphik 12: Harnsäurewerte im Vergleich zwischen den Gruppen. Gruppe A $(n=30)$ und Gruppe B $(n=30)$, Angabe der Mittelwerte in $\mathbf{m g} / \mathbf{d l}$. 


\section{De-Ritis-Quotient im Gruppenvergleich}

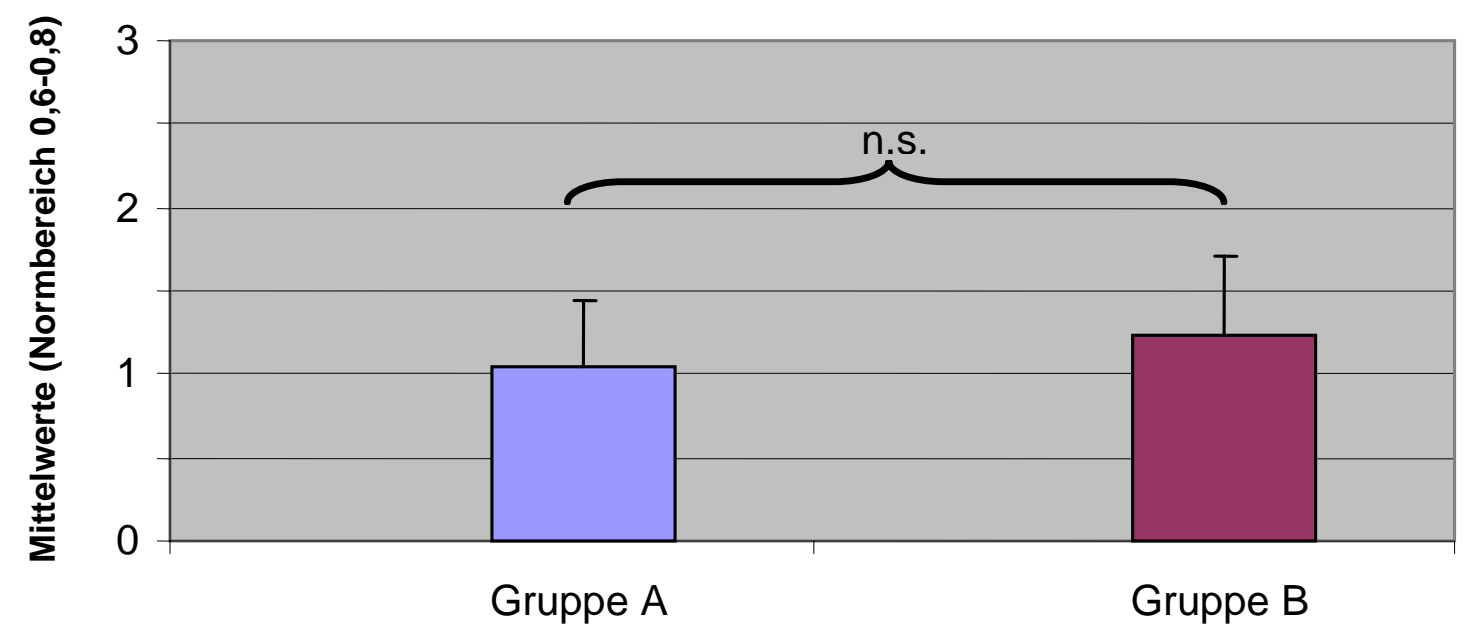

Graphik 13: De-Ritis-Quotienten im Vergleich zwischen den Gruppen. Gruppe A (n=30) und Gruppe B $(n=30)$, Angabe der Mittelwerte in U/l.

\subsection{Adomensonographische Befunde}

Die Häufigkeit des Auftretens pathologischer abdomensonographischer Befunde wurde geprüft und zwischen den Gruppen verglichen. Graphik 14 zeigt die Anzahl der Patienten mit bildgebenden Veränderungen.

\section{Abdomensonographie im Gruppenvergleich}

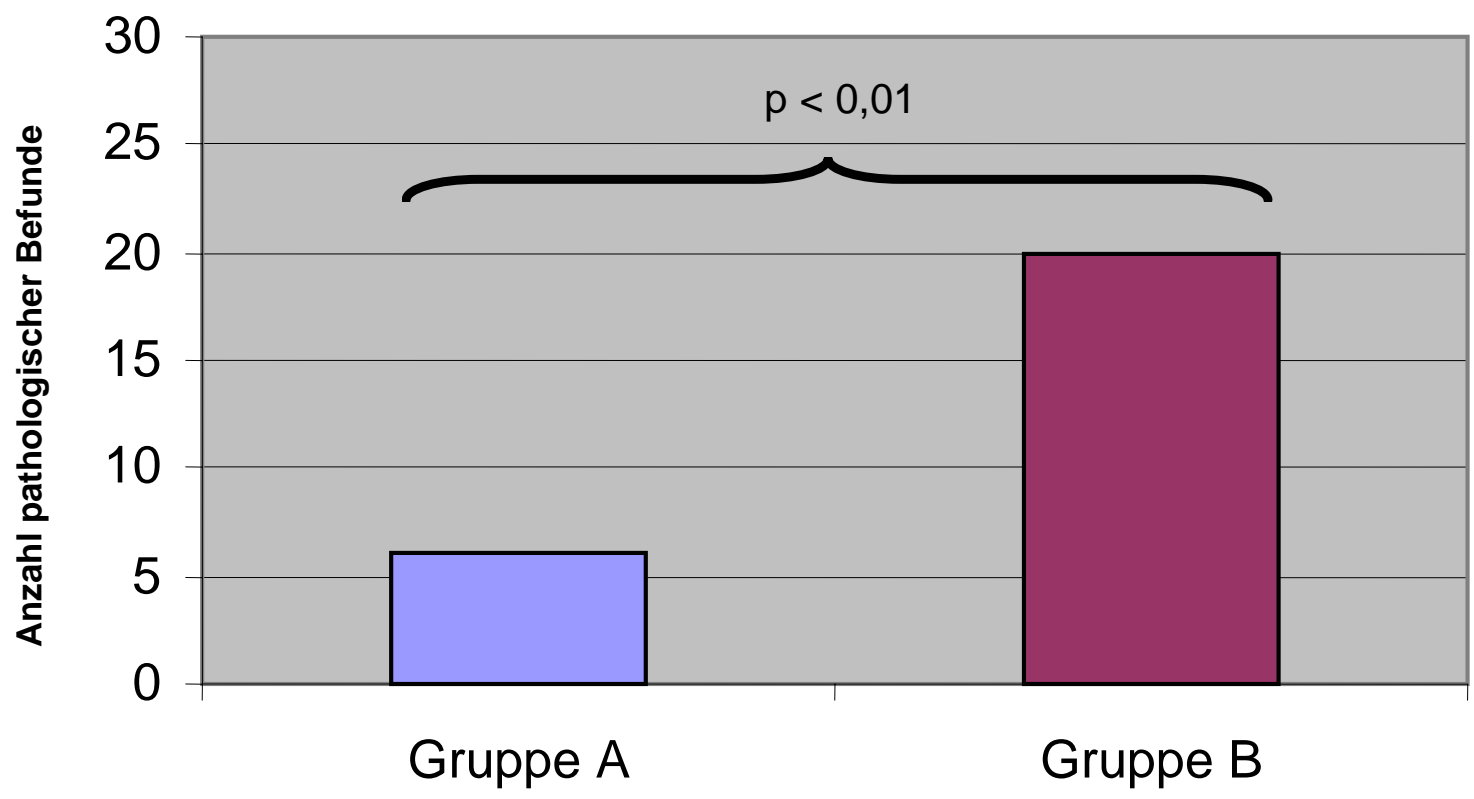

Graphik 14: Abdomensonographische Befunde im Vergleich zwischen den Gruppen. In Gruppe A (n=28) und Gruppe B (n=27), Angabe in Anzahl der Patienten. 
Die abdomensonographisch erhobenen Befunde wurden aufgrund der nominalverteilten Daten mittels Chi-Quadrat-Tests ausgewertet. Es ergab sich ein signifikant häufigeres Auftreten $(\mathrm{p}<0,01)$ von bildgebenden Veränderungen wie Steatosis hepatis, Hepatomegalie, Splenomegalie, Aszites und Leberzirrhose in Gruppe B, passend zu den Befunden der Leberwerte.

\subsection{Bildgebende pathologische Befunde des Neurokraniums}

Weiterhin wurde die Häufigkeit des Auftretens pathologischer Befunde des Neurokraniums zwischen den Gruppen verglichen. Im Chi-Quadrat-Test ergaben sich hierbei keine signifikanten Unterschiede in der Häufigkeit des Auftretens von hirnatrophischen Veränderungen ( $p=0,248$; n.s.) und makroangiopathischen Veränderungen ( $p=0,89$; n.s.). Auch das Auftreten von Marklagerläsionen zeigte keine signifikanten Gruppenunterschiede ( $\mathrm{p}=0,06$; n.s.). Mikroangiopathische Veränderungen zeigten sich dagegen signifikant häufiger in Gruppe A $(\mathrm{p}<0,01)$. Einschränkend muss angemerkt werden, dass nur in ca. zwei Drittel der Fälle bildgebende Befunde vorlagen $(n=42)$.

\subsection{EEG-Befunde}

Im Vergleich der Häufigkeit des Auftretens pathologischer EEG-Befunde zeigten sich ebenfalls keine signifikanten Gruppenunterschiede ( $p=0,2$; n.s.) im Chi-Quadrat-Test.

\subsection{Entzugskrampfanfälle}

Entzugskrampfanfälle traten in Gruppe A ( $n=27)$ in 3,7\% der Fälle und in Gruppe B (n=28) in 28,3\% der Fälle auf. Somit traten signifikant häufiger Entzugskrampfanfälle in Gruppe B auf ( $\mathrm{p}=0,013)$, was im Kollektiv der zusätzlich alkoholabhängigen Patienten verständlich erscheint.

\subsection{Zusammenfassung der Vergleiche zwischen den Gruppen}

Somit zeigte sich Hypothese 1 in Bezug auf das Auftreten von somatischen komorbiden Störungsbildern teilweise positiv. Signifikante Unterschiede zwischen den Gruppen zeigten sich im Grad der Erhöhung der Transaminasen, im Rahmen von pathologischen SonoAbdomen-Befunden und Entzugskrampfanfällen. Alle diese Parameter zeigten in Gruppe B einen höheren Schweregrad. Da diese Gruppe die zusätzlich alkoholabhängigen Patienten beinhaltet, werteten wir dies als zu erwartendes Geschehen. Signifikante Unterschiede zwischen anderen somatischen komorbiden Erkrankungen zeigten sich nicht. 


\subsubsection{Hypothese 2: Benzodiazepinabhängige Patienten haben bei Komorbidität mit einer Alkoholabhängigkeit im Benzodiazepin- entzug ein höheres Ausmaß an kognitiven Defiziten als isoliert benzodiazepinabhängige Patienten}

Das Ausmaß kognitiver Defizite im Benzodiazepinentzug wurde mittels Uhrentest und MMST geprüft und zwischen den Gruppen verglichen.

\subsubsection{Vergleich kognitiver Defizite im Uhrentest (CDT)}

Die folgende Graphik 15 zeigt die Ergebnisse des Uhrentest nach SHULMAN (1993) im Gruppenvergleich angegeben in Prozent der Patienten.

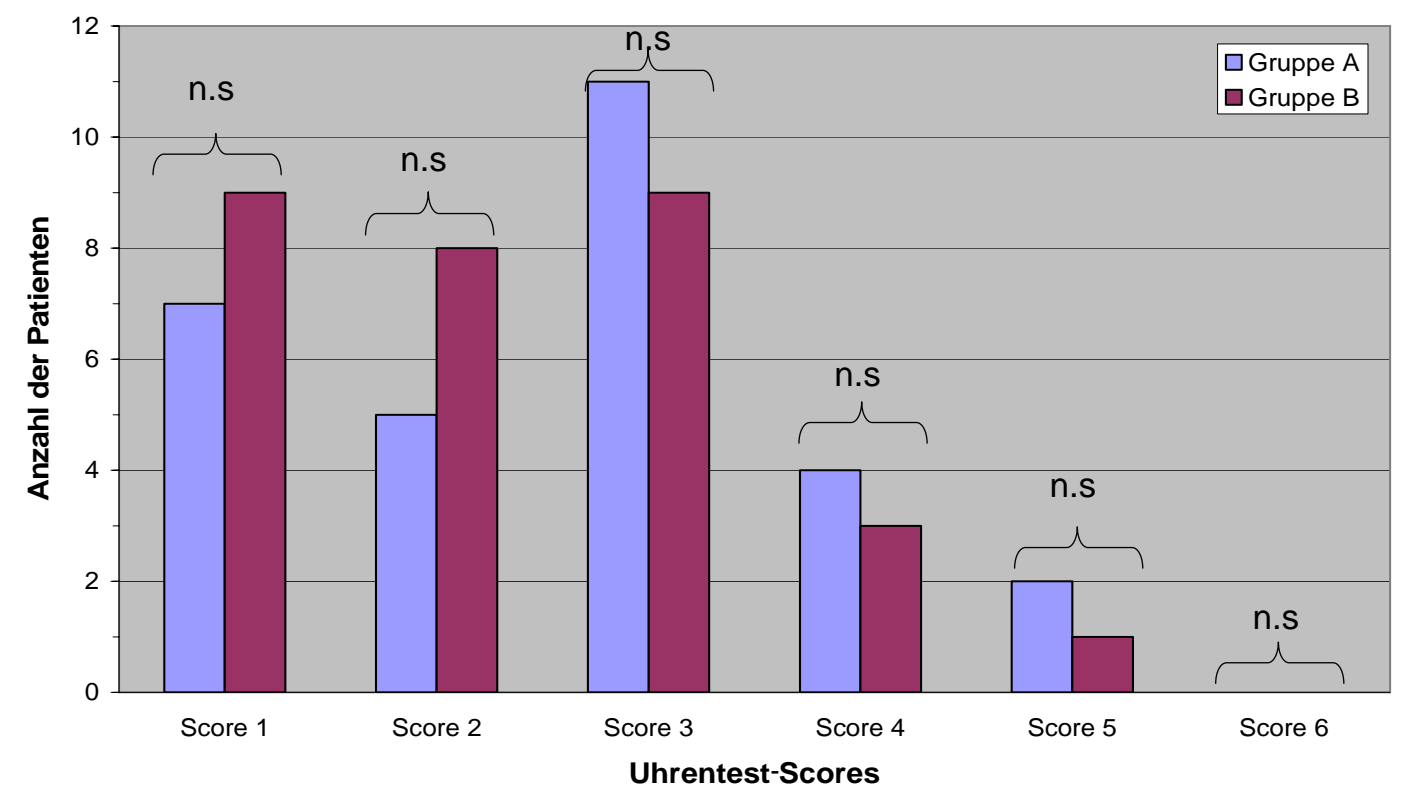

Graphik 15: Ergebnisse des Uhrentest im Vergleich zwischen den Gruppen. Gruppe A (n=29) und Gruppe B (n=30), Angabe in Anzahl der Patienten.

Im Vergleich der Ergebnisse des Uhrentest ergaben sich im Mann-Whitney-U-Test trotz nomineller Unterschiede keine signifikanten Unterschiede zwischen den Gruppen (U=368; $\mathrm{p}=0,21 ;$ n.s.).

\subsubsection{Vergleich kognitiver Defizite im MMST}

Auch der Vergleich des MMST nach FOLSTEIN et al. (1975) zeigte keinen signifikanten Unterschied ( $U=395 ; p=0,4$; n.s.). 


\subsection{Zusammenfassung der Vergleiche zwischen den Gruppen}

Hypothese 2 konnte somit nicht bestätigt werden. Es fanden sich keine Anhaltspunkte für Unterschiede kognitiver Defizite zwischen beiden Gruppen, bei jedoch schlechtem Ausgangsniveau in beiden Gruppen.

Bei einem hohen Anteil an Somatisierungsstörungen in Gruppe A verglichen wir weiterhin die Ergebnisse der Uhrentests von Patienten mit Somatisierungsstörungen mit denen der Patienten ohne Somatisierungsstörungen. Graphik 16 zeigt den Vergleich der Mittelwerte der Uhrentest-Scores von Patienten mit und ohne Somatisierungsstörungen.

Insgesamt ergaben sich keine signifikanten Gruppenunterschiede bei etwas schlechteren Durchschnittswerten der Patienten ohne Somatisierungsstörung.

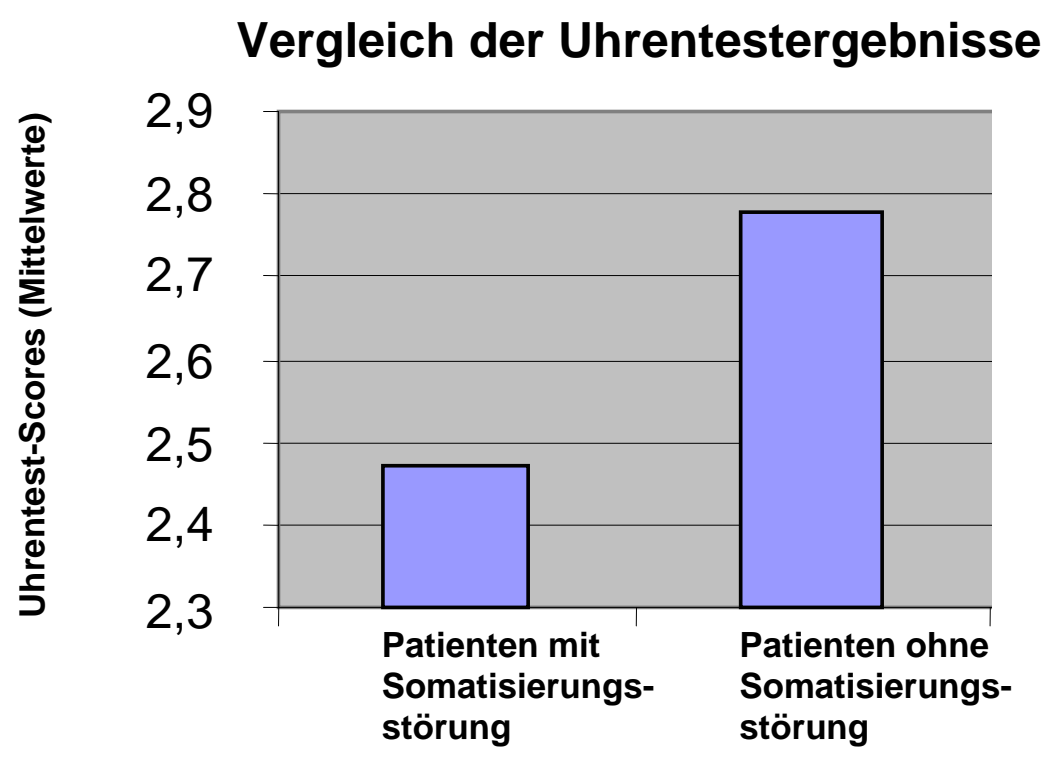

Graphik 16: Vergleich der Uhrentest-Scores angegeben in Mittelwerten von Patienten mit Somatisierungsstörungen $(n=15)$ und ohne Somatisierungsstörungen $(n=15)$ in Gruppe A 


\subsubsection{Hypothese 3: Benzodiazepinabhängige Patienten unterscheiden sich von benzodiazepin- und alkoholabhängigen Patienten im Benzodiazepinentzug hinsichtlich des Schweregrades der Abhängigkeit}

Zur Überprüfung signifikanter Unterschiede im Schweregrad der Abhängigkeit wurden die ASI-Scores und Schweregrade des Suchtbereiches (Drogengebrauch 1, Drogengebrauch 2) und Schweregrade der Abhängigkeit, die Entzugsstartdosis und die Mittelwerte der RickelsScores beider Gruppen miteinander verglichen.

Graphik 17 zeigt die ASI-Scores zum Schweregrad der Abhängigkeit angegeben als Mittelwerte.

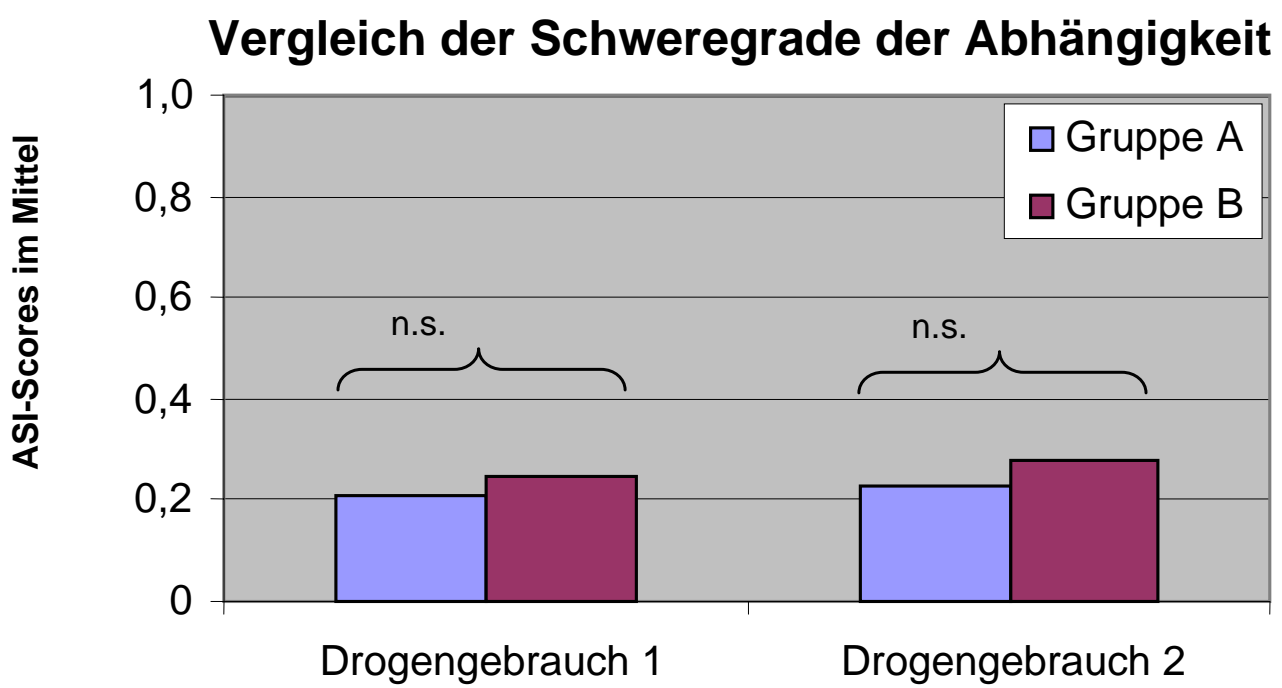

Graphik 17: ASI-Scores zum Schweregrad der Abhängigkeit. Angabe als Mittelwerte Gruppe A (n=30) und Gruppe B $(\mathbf{n}=30)$.

Im Vergleich der ASI-Scores Drogengebrauch 1 (U=380,00; $\mathrm{p}=0,30$; n.s.) und Drogengebrauch 2 ( $U=319,50 ; p=0,053 ; n . s)$ sowie in den Schweregraden des Drogengebrauchs (U=440,5; p=0,88; n.s), zeigte sich im Mann-Whitney-U-Test kein signifikanter Unterschied zwischen beiden Gruppen.

Im Vergleich der Entzugsstartdosen ( $U=379,00 ; p=0,665$; n.s.) ließen sich ebenfalls keine signifikanten Unterschiede zwischen den Gruppen nachweisen.

Auch im Vergleich der Mittelwerte der Rickels-Scores ( $U=111,50 ; p=0,433$; n.s) zeigten sich keine signifikanten Gruppenunterschiede. Es muss bei den Rickels-Scores allerdings die unvollständige Teilnehmerzahl mitberücksichtigt werden (Gruppe 1: n=17, Gruppe 2 n=15). 
Hypothese 3 konnte somit nicht bestätigt werden. In unserer Studiengruppe zeigte sich kein Unterschied des Schweregrades der Abhängigkeit im Vergleich zwischen isoliert benzodiazepinabhängigen Patienten und benzodiazepin- und alkoholabhängigen Patienten.

Im Folgenden wurde der Zusammenhang zwischen dem Schweregrad der Abhängigkeit und dem Vorhandensein von psychiatrischen und somatischen Komorbiditäten, kognitiven Defiziten und der Anzahl im Vorfeld stattgefundener Entzugsbehandlungen geprüft. Zur Beurteilung des Schweregrades der Abhängigkeit wurden die oben bereits genannten Parameter ASI-Scores und Schweregrade des Suchtbereiches (Drogengebrauch 1, Drogengebrauch 2 und Schweregrad der Abhängigkeit), die Entzugsstartdosis und die Mittelwerte der Rickels-Scores verwendet.

Wir verwendeten den Spearman-Test zur Berechnung statistisch signifikanter Korrelationen.

\subsubsection{Hypothese 3.1: Ein höherer Schweregrad der Abhängigkeit korreliert mit dem Vorhandensein von psychiatrischen und somatischen Komorbiditäten}

\subsection{Korrelation Schweregrad der Abhängigkeit/ psychiatrische Komorbiditäten}

Wir setzten die Parameter des Schweregrades der Abhängigkeit in Zusammenhang mit psychiatrischen komorbiden Störungsbildern. Der Vergleich erfolgte mit den im Mini-Dips erfassten Störungsbildern aus den Bereichen der Angsstörungen, posttraumatischen Belastungsreaktionen, affektiven Störungen, somatoformen Störungen, Essstörungen, und Psychosen.

Im Vergleich der Entzugsstartdosen mit den psychiatrischen Störungsbildern ergaben sich keine signifikanten positiven Korrelationen.

Auch die Höhe der ASI-Scores zeigte keinen signifikanten Zusammenhang zur Häufigkeit des Auftretens psychiatrischer Störungsbilder. Der Vergleich der ASI-Schweregrade zeigte dagegen in Gruppe A einen negativen Zusammenhang zum Auftreten von posttraumatischen Belastungsstörungen. Patienten mit hohen ASI-Scores zeigten signifikant weniger PTBS $(\mathrm{r}=-0,373 ; \mathrm{p}<0,05)$. In Gruppe $\mathrm{B}$ zeigte sich kein Zusammenhang $\mathrm{zu}$ psychiatrischen Störungsbildern.

Die Höhe der Rickels-Scores ergab in beiden Gruppen keine signifikanten Korrelationen in Bezug auf das Auftreten psychiatrischer Störungsbilder.

Auch Hypothese 3.1 konnte somit weitestgehend nicht bestätgt werden. Es zeigte sich lediglich ein negativer Zusammenhang zwischen hohen Schweregraden der Abhängigkeit und PTBS in Gruppe A. 


\subsection{Korrelation des Schweregrades der Abhängigkeit mit somatischen Komorbiditäten}

Die oben genannten Parameter des Schweregrades der Abhängigkeit wurden mit komorbiden somatischen Begleiterkrankungen, Leberwerten, abdomensonographischen Befunden, pathologischen bildgebenden Befunden und EEG-Befunden in Verbindung gesetzt.

Tabelle 14 zeigt eine Übersicht der signifikanten Korrelationen. Wir prüften den Zusammenhang zwischen der Höhe der Entzugsstartdosis und dem Auftreten pathologischer somatischer Befunde. Im Spearman-Test zeigten sich in keiner der beiden Gruppen signifikante Korrelationen. Weiterhin verglichen wir die ASI-Gesamtscores aller Problembereiche mit den somatischen Befunden.

Tabelle 14: Signifikante Korrelationen im Vergleich der Parameter der Schwere der Abhängigkeit mit somatischen Komorbiditäten. Gruppe A $(n=30)$, Gruppe B $(n=30)$.

\begin{tabular}{|c|c|c|}
\hline & \multicolumn{2}{|c|}{$\begin{array}{c}\text { Somatische Komorbiditäten } \\
\text { (Laborwerte, bildgebende-Befunde, EEG, somatische Erkrankungen) }\end{array}$} \\
\hline & $\begin{array}{l}\text { Signifikante Korrelationen } \\
\text { Gruppe A }(n=30)\end{array}$ & $\begin{array}{l}\text { Signifikante Korrelationen } \\
\text { Gruppe B }(n=30)\end{array}$ \\
\hline $\begin{array}{l}\text { Höhe der } \\
\text { Entzugsstartdosis }\end{array}$ & Keine & Keine \\
\hline $\begin{array}{l}\text { Höhe der } \\
\text { ASI-Scores }\end{array}$ & Keine & $\begin{array}{l}\text { Positive Korrelation mit der Höhe } \\
\text { der gamma-GT }(r=0,383 ; p<0,05)\end{array}$ \\
\hline \begin{tabular}{|l|} 
Höhe der \\
ASI-Schweregrade
\end{tabular} & $\begin{array}{l}\text { Negative Korrelation mit der Anzahl } \\
\text { degenerativer Erkrankungen des } \\
\text { Bewegungsapparates }(r=0,398 ; p<0,05)\end{array}$ & $\begin{array}{l}\text { Positive Korrelation mit der Höhe } \\
\text { der gamma-GT }(r=0,398 ; p<0,05) \\
\text { Positive Korrelation mit der Höhe } \\
\text { der gamma-GT }(r=0,368 ; p<0,05) \\
\text { Positive Korrelation mit der Höhe } \\
\text { der gamma-GT }(r=0,394 ; p<0,05)\end{array}$ \\
\hline \begin{tabular}{|l|} 
Höhe der \\
Rickels-Scores
\end{tabular} & $\begin{array}{l}\text { Negative Korrelation mit der Anzahl von } \\
\text { Herz-Kreislauf-Erkrankungen }(r=0,398 ; \\
p<0,05)\end{array}$ & $\begin{array}{l}\text { Positive Korrelation mit der Höhe des } \\
\text { De-Ritis-Quotienten }(r=0,677, p<0,01) \\
\text { Positive Korrelation mit der Anzahl } \\
\text { hirnatrophischer Veränderungen } \\
(r=0,394 ; p<0,05)\end{array}$ \\
\hline
\end{tabular}

In Gruppe A zeigte sich kein Zusammenhang zwischen der Höhe der ASI-Scores und somatischen Befunden. In Gruppe B ergab sich ein positiver Zusammenhang zu den dokumentierten Laborwerten. Patienten mit hohen ASI-Scores zeigten signifikant höhere Werte des Leberenzyms gamma-GT $(\mathrm{r}=0,383, \mathrm{p}<0,05)$. Auch die Gesamtwerte der ASISchweregrade wurden in Bezug zu den somatischen Befunden gesetzt. In Gruppe A ergab sich ein negativer Zusammenhang zu degenerativen Erkrankungen des Bewegungsapparates. Bei höheren Schweregraden im ASI zeigten die Patienten signifikant weniger degenerative Erkrankungen des Bewegungsapparates $(r=-0,425, p<0,05)$. In Gruppe B ergab sich im 
Vergleich der ASI-Schweregrade mit den somatischen Befunden, genau wie im Vergleich der ASI-Scores mit den somatischen Befunden, ein positiver Zusammenhang zu den gemessenen Laborwerten. Die Höhe der Schweregrade korrelierte signifikant positiv mit erhöhten Werten von gamma-GT $(r=0,398 ; \mathrm{p}<0,05)$ und GOT $(0,368 ; \mathrm{p}<0,05)$. Zusätzlich zeigte sich im Zusammenhang von ASI-Schweregraden und sonographischen Befunden ein signifikant häufigeres Auftreten pathologischer Leberbefunde $(r=0,394$; $\mathrm{p}<0,05)$ bei hohen ASI-Werten.

Die Gesamtwerte der Rickels-Scores als Parameter vorhandener Entzugssymptomatik wurden in Zusammenhang mit den somatischen Befunden gesetzt. In Gruppe A stellte sich ein negativer Zusammenhang zu vorhandenen Herz-Kreislauf-Erkrankungen dar. Patienten mit stärkerer Entzugssymptomatik wiesen signifikant weniger Herz-Kreislauf-Erkrankungen auf $(\mathrm{r}=-0,459 ; \mathrm{p}<0,05)$. In Gruppe B zeigte der Vergleich von Rickels-Scores und Laborwerten einen positiven Zusammenhang zum De-Ritis-Quotienten. Patienten mit schwerer Entzugssymptomatik wiesen einen hochsignifikant höheren De-Ritis-Quotienten auf ( $\mathrm{r}=0,677$; $\mathrm{p}<0,01$ ). Der Vergleich der Rickels-Scores mit bildgebenden Veränderungen zeigte ein hochsignifikant häufigeres Auftreten von hirnatrophischen Veränderungen im CT/ MRT ( $\mathrm{r}=0,826 ; \mathrm{p}<0,01)$ bei hohen Rickels-Scores.

Hypothese 3.1 konnte insbesondere in Gruppe B bestätigt werden, wo sich positive Korrelationen zwischen den verschiedenen Parametern des Schweregrades der Abhängigkeit und pathologisch erhöhten Leberwerten bzw. De-Ritis-Quotienten und hirnatrophischen Veränderungen zeigten. In Gruppe A zeigten sich lediglich negative Korrelationen zwischen den Parametern der Abhängigkeit und degenerativen Veränderungen des Bewegungsapparates bzw. Herz-Kreislauf-Erkrankungen.

\subsubsection{Hypothese 3.2: Ein höherer Schweregrad der Abhängigkeit korreliert mit einem höheren Ausmaß an kognitiven Defiziten im Benzodiazepinentzug}

Hierbei wurden die oben genannten Parameter der Schwere der Abhängigkeit mit den Ergebnissen des MMST und des Uhrentests verglichen. Im Spearman-Test konnten keine signifikanten Korrelationen zwischen der Höhe der Entzugsstartdosis, der Höhe der ASIScores und der Schweregrade, der Höhe der Rickels-Scores und den im Uhrentest und MMST erhobenen Parametern nachgewiesen werden. Es zeigten sich in drei Korrelationen allerdings Trends, die wir aufgrund der multiplen Korrelationsrechnungen nicht weiter verfolgten.

Tabelle 15 zeigt eine Übersicht der Korrelationen. Hypothese 3.2 konnte somit nicht bestätigt werden. Es zeigten sich keine signifikanten Zusammenhänge zwischen einem höheren Schweregrad der Abhängigkeit und kognitiven Defiziten. 
Weiterhin wurde geprüft ob ein Zusammenhang zwischen kognitiven Defiziten und komorbiden psychiatrischen Erkrankungen besteht. Dies bestätigte sich nicht.

Tabelle 15: Korrelationen (r) mit Wahrscheinlichkeit (p) im Vergleich der Parameter der Schwere der Abhängigkeit mit den Ergebnissen des Mini Mental Status Test und der Uhrentests. Gruppe A (n=30), Gruppe B (n=30).

\begin{tabular}{|c|c|c|c|c|c|c|c|c|}
\hline \multirow{3}{*}{ Signifikante Korrelationen } & \multicolumn{8}{|c|}{$\begin{array}{l}\text { Kognitive Defizite } \\
\text { Mini Mental Status Test }\end{array}$} \\
\hline & \multicolumn{2}{|c|}{ Gruppe A } & \multicolumn{2}{|c|}{ Gruppe B } & \multicolumn{2}{|c|}{ Gruppe A } & \multicolumn{2}{|c|}{ Gruppe B } \\
\hline & $\mathbf{r}$ & p & $\mathbf{r}$ & $\mathbf{p}$ & $\mathbf{r}$ & p & $\mathbf{r}$ & $\mathbf{P}$ \\
\hline Höhe der Entzugsstartdosis & $-0,138$ & 0,483 & 0,221 & 0,248 & $-0,361$ & 0,064 & $-0,108$ & 0,576 \\
\hline Höhe der ASI-Scores & 0,095 & 0,618 & $-0,076$ & 0,690 & 0,096 & 0,619 & 0,354 & 0,055 \\
\hline Höhe der ASI-Schweregrade & 0,099 & 0,603 & 0,195 & 0,603 & 0,029 & 0,880 & 0,168 & 0,376 \\
\hline Höhe der Rickels-Scores & 0,428 & 0,067 & 0,194 & 0,506 & 0,512 & 0,303 & 0,020 & 0,947 \\
\hline
\end{tabular}

Die folgende Tabelle 16 zeigt einen Vergleich der Ergebnisse der MMSTs und Uhrentests von Patienten mit komorbiden psychiatrischen Erkrankungen und ohne komorbide psychiatrische Erkrankungen.

Tabelle 16: Vergleich der Ergebnisse von MMST und Uhrentest von Patienten mit komorbiden psychiatrischen Erkrankungen und ohne komorbide psychiatrische Erkrankungen.

\begin{tabular}{|l|c|c|}
\hline & $\begin{array}{c}\text { MMST } \\
\text { (Mittelwerte) }\end{array}$ & $\begin{array}{c}\text { Uhrentest } \\
\text { (Mittelwerte) }\end{array}$ \\
\hline Affektive Störung & 28,29 & 2,46 \\
\hline Keine Affektive Störung & 28,33 & 2,45 \\
\hline Angststörung & 28,40 & 2,43 \\
\hline Keine Angststörung & 27,92 & 2,53 \\
\hline Somatisierungsstörung & 28,41 & 2,47 \\
\hline Keine Somatisierungsstörung & 28,25 & 2,45 \\
\hline
\end{tabular}

\subsubsection{Hypothese 3.3: Ein höherer Schweregrad der Abhängigkeit korreliert mit der Anzahl bisheriger Entgiftungsbehandlungen}

Hierbei wurden die oben genannten Parameter des Schweregrades der Abhängigkeit in Verbindung mit der Anzahl bisher erfolgter Entgiftungsbehandlungen gesetzt. Im SpearmanTest zeigten sich in keiner der beiden Gruppen signifikante Korrelationen zwischen dem Schweregrad der Abhängigkeit und der Anzahl stattgefundener Entgiftungen. Somit stand der Schweregrad der Abhängigkeit in unserer Studiengruppe in keinem Zusammenhang mit bisher erfolgten Entgiftungensbehandlungen. Tabelle 17 zeigt eine Übersicht der Korrelationen. Auch Hypothese 3.3 bestätigte sich nicht. Es bestand kein Zusammenhang zwischen dem Schweregrad der Abhängigkeit und der Anzahl bisheriger Entgiftungsbehandlungen. 
Tabelle 17: Korrelationen (r) mit Wahrscheinlichkeit (p) im Vergleich der Parameter der Schwere der Abhängigkeit mit der Anzahl stattgefundener Entgiftungsbehandlungen. Gruppe A (n=30), Gruppe B $(\mathbf{n}=30)$.

\begin{tabular}{|l|r|r|r|r|}
\hline \multirow{2}{*}{ Korrelationen } & \multicolumn{4}{|c|}{ Entgiftungsbehandlungen im Vorfeld } \\
\cline { 2 - 5 } & \multicolumn{2}{|c|}{ Gruppe A } & \multicolumn{2}{c|}{ Gruppe B } \\
\cline { 2 - 5 } & $\mathbf{r}$ & \multicolumn{1}{c|}{$\mathbf{p}$} & $\mathbf{r}$ \\
\hline Höhe der Entzugsstartdosis & 0,206 & 0,294 & 0,132 & 0,493 \\
\hline Höhe der ASI-Scores & 0,040 & 0,833 & $-0,163$ & 0,390 \\
\hline Höhe der ASI-Schweregrade & 0,124 & 0,515 & 0,033 & 0,861 \\
\hline Höhe der Rickels-Scores & $-0,120$ & 0,626 & 0,034 & 0,908 \\
\hline
\end{tabular}

\subsubsection{Hypothese 3.4: Das Vorhandensein psychiatrischer Komorbiditäten korreliert mit der Anzahl bisheriger Entgiftungsbehandlungen}

Im Folgenden prüften wir den Zusammenhang zwischen der Anzahl im Vorfeld erfolgter Entgiftungen und der Häufigkeit psychiatrischer Komorbiditäten.

Verglichen wurde dabei die Anzahl der bisher erfolgten Entgiftungsbehandlungen mit den im Mini-Dips erfassten Störungsbildern aus den Bereichen der Angsstörungen, posttraumatischen Belastungsreaktionen, affektiven Störungen, somatoformen Störungen, Essstörungen, und Psychosen. In Gruppe A korrelierte die Anzahl bisheriger Entgiftungsbehandlungen im Spearman-Test negativ mit der Häufigkeit affektiver Störungsbilder. Patienten mit mehr im Vorfeld stattgefundener Entgiftungen zeigten signifikant weniger affektive Störungen $(\mathrm{r}=-0,414 ; \mathrm{p}<0,05)$. In Gruppe B zeigte sich kein Zusammenhang zwischen der Anzahl der Entgiftungsbehandlungen und dem Auftreten psychischer Störungsbilder (vgl. Tabelle 18).

Hypothese 3.4 bestätigte sich teilweise. In Gruppe A zeigten Patienten mit mehr im Vorfeld erfolgten Entgiftungen signifikant weniger affektive Störungen. In Gruppe B zeigte sich kein Zusammenhang.

Tabelle 18: Korrelationen im Vergleich des Vorhandenseins von psychiatrischen Komorbiditäten mit der Anzahl stattgefundener Entgiftungsbehandlungen. Gruppe A $(n=30)$, Gruppe B (n=30).

\begin{tabular}{|l|r|r|r|r|}
\hline & \multicolumn{4}{l|}{ Entgiftungsbehandlungen im Vorfeld } \\
\hline & \multicolumn{2}{l|}{$\begin{array}{l}\text { Signifikante Korrelationen } \\
\text { Gruppe A }\end{array}$} & \multicolumn{1}{l|}{$\begin{array}{l}\text { Signifikante Korrelationen } \\
\text { Gruppe B }\end{array}$} \\
\hline & $\mathbf{r}$ & \multicolumn{1}{c|}{$\mathbf{p}$} & $\mathbf{r}$ & \multicolumn{1}{c|}{$\mathbf{p}$} \\
\hline Affektive Störungen & $-0,414$ & 0,023 & 0,171 & 0,365 \\
\hline Angststörungen & 0,058 & 0,760 & 0,021 & 0,913 \\
\hline Somatoforme Störungen & 0,178 & 0,346 & 0,085 & 0,654 \\
\hline PTBS & 0,069 & 0,717 & 0,026 & 0,890 \\
\hline Essstörungen & 0,021 & 0,913 & $-0,239$ & 0,204 \\
\hline Psychosen & 0,243 & 0,196 & $-0,225$ & 0,232 \\
\hline
\end{tabular}




\subsubsection{Hypothese 3.5: Ein höherer ASI-Score des Problembereiches „körperlicher Zustand“ korreliert mit erhöhten Laborwerten, pathologischen Abdomen- sonographie-Befunden, cCT-, c-MRT-Befunden, EEG-Befunden oder anderen somatischen Begleiterkrankungen}

Weiterhin erfolgte die Gegenüberstellung von ASI-Scores und Schweregraden des Problembereichs „körperlicher Zustand“ und somatischen Befunden.

Wir verglichen die ASI-Scores und Schweregrade des Bereichs „körperlicher Zustand“ mit den oben bereits aufgeführten somatischen Parametern wie Laborwerten, bildgebenden Befunden, EEG-Befunden und somatischen Begleiterkrankungen.

Der Vergleich der ASI-Scores des Problembereichs „körperlicher Zustand“ mit den somatischen Befunden ergab in Gruppe A ein signifikant niedrigeres Auftreten von chronischen Schmerzsyndromen bei höheren ASI-Scores $(r=-0,406 ; p<0,05)$ und ASISchweregraden $(r=-0,459 ; \mathrm{p}<0,05)$. Dieses Ergebnis zeigte sich auch in Gruppe B mit einem hier hochsignifikant selteneren Auftreten von chronischen Schmerzsyndromen bei höheren ASI-Scores ( $r=-0,645 ; \mathrm{p}<0,01)$ und ASI-Schweregraden $(\mathrm{r}=-0,670 ; \mathrm{p}<0,01)$.

In Gruppe B ergab sich zusätzlich ein signifikant selteneres Auftreten von degenerativen Erkrankungen des Bewegungsapparates $(r=-0,393$; $\mathrm{p}<0,05)$ bei Patienten mit höheren ASIScores $(r=-0,393 ; p<0,05)$ und ASI-Schweregraden $(r=-0,368 ; p<0,05)$.

Alle weiteren Parameter korrelierten im Spearman-Test nicht.

Hypothese 3.5 bestätigte sich. In beiden Gruppen ergab sich ein signifikant niedrigeres Auftreten von chronischen Schmerzsyndromen bei Patienten mit hohen ASI-Scores. In Gruppe B bestand zusätzlich ein negativer Zusammenhang mit chronischen Schmerzsyndromen und hohen ASI-Scores. 


\section{Diskussion}

\subsection{Soziodemographische Gesichtspunkte:}

Zunächst erfolgt eine Beschreibung soziodemographischer Parameter des gesamten Studienkollektivs. An der in Göttingen durchgeführten Studie nahmen sechzig Probanden teil. Dreißig Probanden befanden sich aufgrund einer reinen Benzodiazepinabhängigkeit in Behandlung, weitere dreißig wurden aufgrund einer Doppelabhängigkeit von Benzodiazepinen und Alkohol behandelt.

Im gesamten Kollektiv zeigte sich ein ausgeglichenes Geschlechterverhältnis mit 46,7\% Frauen und 53,3\% Männern. Diese Verteilung wurde bewusst herbeigeführt, um eine bessere Beurteilbarkeit zu errreichen. In der Normalbevölkerung sind zu einem höheren Anteil, von ca. zwei Dritteln, Frauen von Medikamentenabhängigkeit betroffenen (POSER W und POSER S 1996).

Das Durchschnittsalter der Patienten lag bei 51,7 Jahren. Dieser Altersdurchschnitt ähnelt im Vorfeld untersuchten Kollektiven (z.B. CHARNEY et al. 2000). Viele Studien beziehen sich jedoch insbesondere auf ältere Patienten, da die Abhängigkeit von Sedativa/Hypnotika überwiegend in der zweiten Lebenshälfte auftritt (POSER W et al. 2006). So besteht eine signifikant höhere langzeitige Anwendung von Benzodiazepinen im Fall älterer Patienten. In der Population der über 65 Jährigen erhalten 15\% regelmäßig Schlafmittel. In Großbritannien betreffen $80 \%$ der für Benzodiazepine ausgestellten Verschreibungen ältere Menschen (CURRAN et al. 2003).

Bei Betrachtung der Arbeitssituation fällt eine deutliche Einschränkung sozialer Funktionen auf. Im gesamten Kollektiv der benzodiazepinabhängigen Patienten waren 26,7\% arbeitslos, nur ungefähr ein Drittel befand sich überhaupt in einem geregelten Arbeitsverhältnis. Auffallend hoch zeigte sich mit 36,7\% das Quantum der Patienten, die vollständig aus dem Arbeitsleben ausgeschieden waren (Rentner/ Frührentner) insbesondere in Bezug auf den Altersdurchschnitt. Diese Ergebnisse decken sich mit der Arbeitssituation benzodiazepinabhängiger Patienten in früher untersuchten Kollektiven. So beschreiben beispielsweise CHARNEY et al. (2000) in ihrer Studie mit benzodiazepinabhängigen Patienten einen Anteil arbeitsloser Probanden von 40\%, während sich nur 28\% in einem geregelten Arbeitsverhältnis befanden. Bereits berentete Patienten wurden in der Studie nicht mit aufgeführt. In der vorliegenden Studie zeigte sich mit 30\% ein größerer Anteil arbeitsloser Patienten in der Gruppe der zusätzlich alkoholabhängigen Patienten. Insgesamt sind alkoholabhängige 
Patienten stärker von Arbeitslosigkeit betroffen als rein benzodiazepinabhängige Patienten. So zeigte beispielsweise eine von SCHNEIDER et al. (2001) durchgeführte Multicenterstudie mit alkoholabhängigen Patienten einen deutlich höheren Anteil arbeitsloser Patienten von 48,3\%. Dort lag der Anteil an berenteten Probanden mit 12,1\% jedoch deutlich niedriger als in der hier vorgestellten Studie. Auch bei den von SCHNEIDER et al. befragten Patienten befanden sich nur etwa 30\% in einem geregelten Arbeitsverhältnis. Der im Vergleich zu vorausgehenden Studien geringere Anteil an arbeitslosen Patienten ist am ehesten bedingt, durch den hohen Anteil an frühen Berentungen in der vorliegenden Studie.

Auch bei Betrachtung partnerschaftlicher Beziehungen zeigten sich im gesamten Kollektiv Probleme mit der Führung stabiler Beziehungen. So war insgesamt ein Anteil von $40 \%$ der Patienten verheiratet, ca. 30\% waren ledig geblieben und 21,7\% waren geschieden. In der Gruppe der zusätzlich alkoholabhängigen Patienten lag die Anzahl der ledig gebliebenen Teilnehmer mit 33,3\% und auch die der geschiedenen Teilnehmer mit 23,3\% etwas höher als in der Gruppe der rein benzodiazepinabhängigen Patienten. Signifikante Gruppenunterschiede ergaben sich aber nicht. Auch hier beschrieben CHARNEY et al. (2000) ähnliche Ergebnisse, wobei der Anteil verheirateter Teilnehmer in ihrer Studie mit benzodiazepinabhängigen Patienten mit 56\% deutlich höher lag. Im Fall alkoholabhängiger Patienten zeigten die Daten von SCHNEIDER et al. (2001) einen sehr niedrigen Anteil verheirateter Patienten von nur 23\%. Analog zu den Daten der vorliegenden Studie lebten 36,4\% der Patienten allein und $37,4 \%$ bereits in Scheidung.

Insgesamt zeigen die Daten der vorliegenden Studie, dass benzodiazepinabhängige Patienten in hohem Maß von Arbeitslosigkeit oder Frühberentungen betroffen sind. Zwar zeigte sich eine deutlichere Ausprägung in der Gruppe der zusätzlich alkoholabhängigen Probanden, in beiden Gruppen waren jedoch nur ca. 30\% der Patienten berufstätig. Weiterhin weisen die Daten, analog zu vorausgehenden Studien mit benzodiazepinabhängigen Patienten (CHARNEY et al. 2000), auf große Probleme mit partnerschaftlichen Beziehungen hin. Nur 40\% der Probanden lebten in einer Partnerschaft.

Bei Betrachtung der verschiedenen Berufe der Studienteilnehmer zeigte sich, dass 33\% der Probanden Angehörige medizinischer Berufsgruppen waren. Dies war sowohl in der Gruppe der zusätzlich alkoholabhängigen Patienten als auch in der Gruppe rein benzodiazepinabhängiger Patienten der Fall. Die Daten gehen dabei konform mit den Ergebnissen anderer Studien. So beschreiben auch KEMPER et al. (1980) ein gehäuftes Auftreten von Benzodiazepinabhängigkeit unter Angehörigen medizinischer Berufe. Dies ist interessant, da gerade in dieser Berufsgruppe ein höherer Wissensstand über mögliche Nebenwirkungen und die 
Entwicklung von Abhängigkeit unter langzeitiger Benzodiazepineinnahme zu erwarten wäre. Insgesamt lässt sich der vermehrte Konsum möglicherweise durch einen erleichterten Zugang zu Medikamenten und eine hohe Stressbelastung erklären.

Die Studie beschreibt im Wesentlichen langzeitabhängige Patienten. Der Einnahmezeitraum der Benzodiazepine lag in der Studiengruppe im Mittel bei ca. 10 Jahren. Im Kollektiv der zusätzlich alkoholabhängigen Patienten wurde im Mittel 14,2 Jahre abhängig getrunken.

Die Patientengruppen unterschieden sich wesentlich in der Anzahl der im Vorfeld stattgefundenen Entgiftungsbehandlungen. In der Gruppe der rein benzodiazepinabhängigen Patienten lag die Anzahl der im Vorfeld erfolgten Entgiftungsbehandlungen im Mittel bei 1,6, in der Gruppe der zusätzlich alkoholabhängigen Patienten jedoch bei 6,6. Die häufigeren Entgiftungen in der letzteren Gruppe sind auf die in diesem Kollektiv stattgefundenen multiplen Alkoholentzüge zurückzuführen.

\subsection{Hypothesen}

Nachfolgend werden die im Vorfeld erstellten Hypothesen auf ihre Gültigkeit überprüft.

\subsubsection{Hypothese 1: Benzodiazepinabhängige Patienten unterscheiden sich von benzodiazepin- und alkoholabhängigen Patienten im Benzodiazepinentzug hinsichtlich psychiatrischer und somatischer Komorbiditäten}

Ein Großteil der vorliegenden Arbeit befasst sich mit der Untersuchung komorbider Störungsbilder benzodiazepinabhängiger Patienten. Die erhobenen Daten wurden im Anschluss mit komorbiden Störungsbildern benzodiazepin- und alkoholabhängiger Patienten verglichen. Während sich bereits viele Studien mit der Komorbidität rein alkoholabhängiger Patienten beschäftigten (SCHNEIDER et al. 2001, SCHMIDT et al. 2003, DRIESSEN et al. 2008), gibt es nur wenige Daten betreffend die Komorbidität benzodiazepinabhängiger Patienten. Das Ziel dieser Studie war es daher, weitere Daten über psychiatrische und somatische Begleiterkrankungen benzodiazepinabhängiger Patienten zu gewinnen. Die Häufigkeit des Auftretens insbesondere von psychiatrischen Begleiterkrankungen bei abhängigen Patienten ist von hohem klinischem Interesse, da diese die Entstehung von Abhängigkeit begünstigen können und die Behandlung und Prognose mitbestimmen. Die Entscheidung der Verschreibung eines Benzodiazepins muss daher von Fall zu Fall sorgfältig erwogen werden unter Berücksichtigung des psychischen Status und des Potentials zur Abhängigkeit. 


\subsubsection{Komorbide psychiatrische Störungsbilder benzodiazepinabhängiger Patienten}

Es folgt die Beschreibung der pychiatrischen komorbiden Störungsbilder des gesamten Patientenkollektivs erfasst durch den Mini-DIPS (nach MARGRAF 1994) und Aktenrecherche.

In der vorliegenden Studie wiesen 85\% der Patienten affektive Störungsbilder auf. Einen Großteil davon machten mit 80\% monopolare Depressionen aus, ein geringer Prozentsatz verteilte sich auf bipolare Störungsbilder. Weiterhin wiesen 78\% der Patienten begleitende Angststörungen auf, vorwiegend Panikstörungen. Somatoforme Störungen zeigten 28,3 \% der Patienten, hierbei wurden insbesondere Somatisierungsstörungen nach den Leitlinien ICD 10 (WHO 1991) beschrieben.

Die Studie zeigt deutlich das hohe Ausmaß komorbider psychiatrischer Erkrankungen langzeitig benzodiazepinabhängiger Patienten. Die Daten beziehen sich dabei nur auf Patienten, die in spezifischen Suchtkliniken behandelt werden. Depressionen und Panikstörungen waren die häufigsten Achse-I-Störungsbilder. Vorausgehende Studien kamen zu ähnlichen Ergebnissen betreffend die Art der komorbiden Störungsbilder, wobei diese in deutlich niedrigeren Prozentzahlen als in der vorliegenden Studie auftraten.

So führten beispielsweise BUSTO et al. (1996) eine Studie zur Komorbidität schwer benzodiazepinabhängiger Patienten mit 30 Teilnehmern im Benzodiazepinentzug durch. Alle befragten Patienten wiesen mindestens ein komorbides Achse-I-Störungsbild auf. In 43\% der Fälle waren dies begleitende depressive Störungsbilder und in 30\% Angsterkrankungen im Sinne von Panikstörungen und generalisierten Angststörungen. Eine klinische Studie von MARTINEZ-CANO et al. (1999) mit 153 benzodiazepinabhängigen Patienten zeigte ebenfalls in erster Line begleitende affektive Störungen und Angststörungen. Auch hier handelte es sich bei den affektiven Störungen vorwiegend um monopolare depressive Störungsbilder und bei den Angststörungen vorwiegend um Panikstörungen. Somatoforme Störungsbilder nach DSM-IV (APA 1994) traten auch bei MARTINEZ-CANO et al. (1999) in einem hohen Prozentsatz auf, wobei es sich hier in erster Linie um Konversionsstörungen handelte, während die Patienten der vorliegenden Studie mehr Somatisierungsstörungen aufwiesen. MERIKANGAS et al. (2008) konnten in einer Studie, die sich auf Daten der „Zurich Cohort Study“ bezog, zeigen, dass Depressionen generell mit einem stark erhöhten Risiko zur Entwicklung einer Benzodiazepinabhängigkeit assoziiert sind. Sie verwiesen hierbei auch auf die Problematik der Verschreibung von Benzodiazepinen, die in über 50\% der Fälle von Patienten mit affektiven Störungen erfolgt und häufig in Missbrauch oder 
Abhängigkeit endet. Auch das Vorhandensein von Panikstörungen scheint insgesamt stark mit einer Substanzabhängigkeit verknüpft. So wird bei diesen Patienten über ein Lebenszeitrisiko zur Entwicklung von Substanzmissbrauch oder Abhängigkeit von über $40 \%$ berichtet (WITTCHEN 1988).

Eine neuere Studie von MOL et al. (2005) mit 193 benzodiazepinabhängigen Patienten im Entzug beschäftigte sich mit dem Zusammenhang zwischen craving und psychopathologischen Veränderungen. Hierbei konnte gezeigt werden, dass Patienten, die ein starkes craving im Sinne von starker Entzugssymptomatik aufwiesen, signifikant mehr affektive Störungen wie Depressionen und zudem Erschöpfung, Ärger und Anspannung zeigten als Patienten, die den Entzug problemfrei durchliefen. Zusätzlich zeigte insbesondere diese Patientengruppe eine hohe Tendenz zur Somatisierung. Die Studie zeigte somit deutlich den Zusammenhang zwischen Somatisierung, Depression und Einnahmegewohnheiten benzodiazepinabhängiger Patienten.

Die Häufigkeit des Auftretens komorbider Störungsbilder in der vorliegenden Studie deutet auf ihre mögliche Rolle als prädisponierende Faktoren zur Entwicklung einer Benzodiazepinabhängigkeit hin. Eine große Rolle scheinen hierbei insbesondere Angst- und depressive Störungen zu spielen. In der Literatur wird der Beginn vieler Probleme mit Drogen oder Alkohol als Resultat des dysfunktionalen Umgangs mit Angst und Depressionen gesehen. Die Betroffenen verwenden die entsprechende Droge oder Alkohol zur Minderung von Symptomen wie Angst oder Traurigkeit, was zu maladaptiven Mechanismen führen kann. So scheint auch Benzodiazepinabhängigkeit bei einigen Patienten ein maladaptiver Mechanismus zu sein oder als Komplikation von Angststörungen oder Depressionen aufzutreten (MARTINEZ-CANO et al. 1999). Insgesamt zeigt der hohe Anteil psychiatrischer Komorbiditäten benzodiazepinabhängiger Patienten die Wichtigkeit der Erkennung selbiger, da sie großen Einfluss auf das Outcome der Patienten haben können.

Spezifische Behandlungen der verschiedenen Krankheitsbilder scheinen notwendig in der Prävention des Auftretens bzw. Wiederauftretens von Substanzmissbrauch. Ob die psychischen Symptome einem Substanzmissbrauch vorausgehen oder in dessen Folge auftreten, bleibt oft unklar. Es wäre wichtig zu ermitteln, wie viele dieser Symptome nach einer Zeit der Abstinenz verschwinden.

Kontrovers wird noch immer die dauerhafte Anwendung von Benzodiazepinen im Fall von Patienten mit chronischen Angsterkrankungen diskutiert. Betrachtet man jedoch die potentiell möglichen, negativen Effekte langjähriger Benzodiazepineinnahme und den Fakt, dass die 
Patienten dauerhaft nicht unbedingt eine Erleichterung ihrer Symptome erfahren, sollte angestrebt werden, auch den Großteil dieser Patienten zu entgiften (CHARNEY et al. 2000).

\subsubsection{Vergleich komorbider psychiatrischer Störungsbilder}

Um oben genannte Hypothese zu prüfen, erfolgte der Vergleich komorbider psychiatrischer Störungsbilder rein benzodiazepinabhängiger und benzodiazepin- und alkoholabhängiger Patienten. Hierbei zeigten sich keine Unterschiede in der Häufigkeit des Auftretens komorbider Angststörungen, posttraumatischer Belastungsstörungen, affektiver Störungen, Essstörungen oder Psychosen zwischen den Gruppen. Somatoforme Störungen traten dagegen hochsignifikant $(\mathrm{p}<0,01)$ häufiger in der Gruppe der isoliert benzodiazepinabhängigen Patienten auf. Somit zeigt sich die Hypothese in Hinsicht auf psychiatrische Störungsbilder bestätigt.

Insgesamt liegen unseres Wissens nach bisher keine Studien über komorbide Störungsbilder sowohl benzodiazepin- als auch alkoholabhängiger Patienten vor.

Eine klinische Studie von CHARNEY et al. (2000) mit 25 benzodiazepinabhängigen und 57 alkoholabhängigen Patienten im Entzug, beschäftigte sich ebenfalls mit der Häufigkeit komorbider Störungsbilder dieser Patienten. Im Vergleich zwischen benzodiazepin- und alkoholabhängigen Patienten zeigte sich in dieser Studie, kontrovers zu den Daten der vorliegenden Studie, ein signifikant häufigeres Auftreten von Angsterkrankungen im Kollektiv der benzodiazepinabhängigen Patienten. Analog der vorliegenden Studie ergab sich jedoch auch hier ein signifikant häufigeres Auftreten von Somatisierungsstörungen im Kollektiv der benzodiazepinabhängigen Patienten im Vergleich zu alkoholabhängigen Patienten. Das Patientenkollektiv scheint jedoch nur sehr eingeschränkt mit dem der vorliegenden Studie vergleichbar, da sich die Studie von CHARNEY et al. (2000) auf rein benzodiazepin- oder alkoholabhängige Patienten bezieht.

Vorhergehende Studien über die Komorbidität rein alkoholabhängiger Patienten zeigten ebenfalls insbesondere das Auftreten von begleitenden affektiven Störungsbildern und Angsterkrankungen. So fanden KESSLER et al. (1997) in einer Studie, die auf Daten des

National Comorbidity Survey mit 8098 Teilnehmern basierte, bei einem Großteil der alkoholabhängigen Patienten mindestens ein komorbides psychiatrisches Störungsbild. Affektive Störungsbilder wiesen hierbei 53,5\% der Frauen und 28,1\% der Männer auf; in erster Linie handelte es sich um monopolare depressive Störungsbilder. Angststörungen zeigten 60,7\% der Frauen und 35,8\% der Männer, am häufigsten traten phobische 
Störungsbilder auf. Dies waren in erster Linie spezifische Phobien wie eine soziale Phobie oder Agoraphobie. Einen weiteren großen Anteil machten PTBS aus.

In der Multi-Center-Studie von SCHNEIDER at al. (2001) mit 556 alkoholabhängigen Probanden zeigten sich in 53,1\% der Fälle Achse-I-Störungsbilder. Es handelte sich hierbei in 42\% der Fälle um Angststörungen, ebenfalls in erster Linie phobische Störungsbilder und in 24\% um affektive Störungen, vorwiegend Depressionen. Eine Studie von GRANT et al. (2004), die sich auf die Ergebnisse des „National Epidemiologic Survey on Alcohol and Related Conditions“ (NESARC) aus den USA (2001-2002) mit 43.093 Teilnehmern bezog, zeigte ebenfalls eine hohe Komorbidität von substanzbedingten Störungen und affektiven sowie Angststörungen. Studienteilnehmer mit einer aktuellen Substanzgebrauchsstörung, die im Befragungszeitraum spezifische Behandlung suchten, wiesen zu 60 \% affektive Störungen und zu 43\% Angsstörungen auf; alkholabhängigen Patienten die spezifische Behandlung ersuchten, zeigten in 40,7 \% der Fälle begleitende affektive und in 33 \% der Fälle begleitende Angststörungen.

Bisher liegen nur wenige Studien vor, die mögliche komorbide somatoforme Störungsbilder substanzabhängiger Patienten evaluiert haben. Diese zeigen außerdem sehr unterschiedliche Ergebnisse. Dennoch tendieren auch die größeren Studien dazu, einen Zusammenhang zwischen somatoformen Störungsbildern und Substanzmissbrauch zu sehen (HASIN und KATZ 2007).

Die Ergebnisse der vorliegenden Studien zeigen die Notwendigkeit, alle Patienten, die sich in Behandlung befinden, genau auf das Vorhandensein psychiatrischer Komorbiditäten zu überprüfen und geeignete Behandlungskonzepte zu entwickeln.

\subsubsection{Komorbide somatische Störungsbilder benzodiazepinabhängiger Patienten}

Es folgt die Beschreibung der somatischen komorbiden Erkrankungen des gesamten Patientenkollektivs erfasst durch Befragung der Patienten und Aktenrecherche.

\subsection{Begleiterkrankungen}

Wir erfassten Begleiterkrankungen wie chronische Schmerzsyndrome, neurologische Erkrankungen, degenerative Veränderungen des Bewegungsapparates, Erkrankungen des Herz-Kreislauf-Systems, arterieller Hypertonus und Stoffwechselerkrankungen. Hierbei konnte gezeigt werden, dass Benzodiazepinabhängigkeit auch häufig mit somatischen Begleiterkrankungen assoziiert ist. 
Es wiesen 48\% der Patienten Erkrankungen des Bewegungsapparates auf und 45\% chronische Schmerzsyndrome. Einen weiteren großen Anteil machten mit 33\% der Untersuchten kardiovaskuläre Erkrankungen und mit 23\% der Untersuchten neurologische Erkrankungen aus.

Vorhergehende Studien, die sich mit begleitenden somatischen Erkrankungen benzodiazepinabhängiger Patienten befassten, kamen zu ähnlichen Ergebnissen. So konnten auch MARTINEZ-CANO et al. (1999) in ihrer Studie einen hohen Anteil an komorbiden rheumatologischen, neurologischen und kardiovaskulären Erkrankungen feststellen.

Hierbei zeigte sich auch ein signifikanter Zusammenhang zwischen rheumatischen Erkrankungen und Schlafstörungen. Da Schlafstörungen einen der häufigsten Gründe einer Benzodiazepineinnahme darstellen, erscheint dies als eine verständliche Ursache der Entwicklung von Abhängigkeit. Ebenso konnten MARTINEZ-CANO et al. (1999) den Zusammenhang zwischen kardiovaskulären Erkrankungen und Angststörungen nachweisen. Auch hier scheint eine somatische Ursache der Auslöser der Entwicklung psychischer Probleme zu sein, die dann wiederum zur Benzodiazepineinnahme führen. Eine große Rolle scheint der oft chronische Verlauf der oben genannten Erkrankungen zu spielen. Auch Patienten mit chronischen Schmerzsyndromen zeigen einen vermehrten Benzodiazepingebrauch. Insbesondere wenn Schmerzsyndrome gemeinsam mit depressiven Episoden auftreten, ist die Wahrscheinlichkeit einer Benzodiazepineinnahme um 62\% gegenüber der Normalbevölkerung erhöht (LIU et al. 2010). Eine Studie von SMOLDERSA et al. (2007) konnte zeigen, dass Angstpatienten mit zusätzlich bestehenden chronischen somatischen Erkrankungen, deutlich häufiger Benzodiazepine verschrieben wurden, als Patienten mit reinen Angsterkrankungen. Auch in der Studie von SMOLDERSA et al. (2007) zeigten sich am häufigsten komorbide Erkrankungen des muskuloskelettalen Systems, kardiologische und neurologische Erkrankungen.

\subsection{Leberwerte und abdomensonographische Befunde}

Es folgt die Beschreibung der Leberwerte und der sonographischen Befunde des Abdomens, die erfasst wurden, um eine mögliche lebertoxische Wirkung der Benzodiazepineinnahme beurteilen zu können. Im Falle der zusätzlich alkoholabhängigen Patienten konnte so das Ausmaß bereits stattgefundener Leberzellschädigung beurteilt werden.

Im Kollektiv der rein benzodiazepinabhängigen Patienten zeigte sich eine leichtgradige Erhöhung der gamma-GT von im Mittel 66,3 U/L. Diese stellt den empfindlichsten Indikator für Störungen der Integrität der Leberzellen und alkoholtoxische Schädigungen dar 
(HEROLD 2008). Auffallend hoch lagen die De-Ritis-Quotienten, die mit dem Umfang der Leberzellschädigung korrelieren (HEROLD 2008), der Wert von im Mittel 1,04 wies hier auf eine mögliche lebertoxische Wirkung der Benzodiazepine hin.

In den sonographischen Befunden des Abdomens zeigten sich in 21,4\% der Fälle Veränderungen wie Steatosis hepatis, Hepatomegalie oder Splenomegalie. Klinisch relevante Veränderungen wie eine Leberzirrhose oder Aszites bestanden nicht.

Trotz der weitverbreiteten Verwendung von Benzodiazepinen, liegen bisher nur wenige Daten zu möglichen hepatotoxischen Nebenwirkungen der langzeitigen Einnahme vor.

Im Fall tierexperimenteller Studien mit Ratten, wird über eine Verschlechterung der Leberfunktion durch langzeitige Einnahme von Diazepam berichtet (KVETINA und GUAITANI 1969). Auch CHATTERJEE et al. (1997) beschreiben mikroskopisch sichtbare pränekrotische und nekrotische Leberveränderungen bei Albinoratten, die über einen Zeitraum von 30 bzw. 60 Tage hochdosiert Diazepam erhielten. Dennoch besteht in tierexperimentellen Studien weiterhin keine Klarheit über die genaue Art möglicher toxischer Veränderungen auf zellulärer und subzellulärer Ebene (CHATTERJEE et al. 1997). Klinische Studien mit menschlichen Probanden, die sich mit einer möglichen hepatotoxischen Wirkung von Benzodiazepinen beschäftigen, liegen bisher nicht vor.

Auch im Falle der vorliegenden Studie bleibt letztendlich unklar, ob die erhöhten Werte von gamma-GT und De-Ritis-Quotient auf die langzeitige Einnahme von Benzodiazepinen zurückzuführen sind.

\subsection{Bildgebende pathologische Befunde des Neurokraniums}

Weiterhin erfassten wir cCT- und cMRT-Befunde des Neurokraniums, um beurteilen zu können ob eine lanjährige Einnahme von Benzodiazepinen auch mit strukturellen Veränderungen des Zentralnervensystems einhergeht.

Obwohl Patienten mit bekannten hirnorganischen Vorschäden oder Demenzen zu Beginn der Studie ausgeschlossen wurden, zeigte sich im gesamten Kollektiv ein hohes Ausmaß an pathologischen Befunden. Im Kollektiv der rein benzodiazepinabhängigen Patienten zeigten sich in erster Linie unspezifische Marklagerläsionen, die bei 31,6\% der Fälle vorlagen. Weiterhin wies ca. ein Viertel der Fälle mikroangiopathische und ein weiteres Viertel hirnatrophische Veränderungen auf. Einschränkend ist anzumerken, dass nur im Fall von 19 der 30 Patienten CT oder MRT Bilder vorlagen.

In der Literatur finden sich widersprüchliche Ergebnisse betreffend einen Zusammenhang zwischen strukturellen, im CT/MRT ersichtlichen Veränderungen des Neurokraniums und 
langzeitiger Benzodiazepineinnahme. So gaben einige Studien eine Zunahme der ventriclebrain-ratio, also einen stärkeren Verlust an Hirnparenchym im Falle langzeitiger Benzodiazepineinnahme an (LADER et al. 1984, SCHMAUSS und KRIEG 1987). Andere Studien sahen dagegen keinen signifikanten Zusammenhang zwischen pathologischen CT-Befunden des Neurokraniums und langzeitiger Benzodiazepineinnahme (POSER W et al. 1983, PERERA et al. 1987, MOODLEY et al. 1993).

SCHWEIZER et al. (1995) beschreiben in einer Übersichtsarbeit der bis 1995 publizierten Studien, dass letztendlich unklar bleibt, ob eine langzeitige Benzodiazepineinnahme zu im CT darstellbaren, pathologischen Hirnveränderungen führen kann. Sie verwiesen hierbei auf methodische Probleme in der Durchführung bisheriger Studien. So wurden komorbide medizinische Faktoren der untersuchten Patienten sowie auch der entsprechenden Kontrollen nicht berücksichtigt. Ob bereits im Vorfeld neurologische Erkrankungen oder ein Missbrauch von Alkohol oder Drogen bestanden, ist nicht dokumentiert. Zudem wiesen viele der untersuchten Patienten komorbide psychiatrische Erkrankungen auf, was die dauerhafte Einnahme weiterer Medikamente sehr wahrscheinlich macht oder selbst die Veränderungen bedingen könnte. Eine neuere Studie von BUSTO et al. (2000), in der Patienten mit komorbiden Störungen ausgeschlossen und alle Patienten mit alters- und geschlechtsgleichen Kontrollen verglichen wurden, ergab keine Hinweise auf pathologische Veränderungen in cCT-Scans dauerhaft benzodiazepinabhängiger Patienten. Der Vergleich zu bisher durchgeführten Studien scheint aber insgesamt schwierig, da sich diese Studien unseres Wissens nach ausschließlich auf Ergebnisse von CT-Scans beziehen. In der vorliegenden Studie wurden jedoch von einem Großteil der Patienten MRT Bilder ausgewertet, die möglicherweise eine genauere Beurteilbarkeit erlaubten. Nach unserem Wissen sind bisher keine entsprechenden Studien mit MRT Aufnahmen durchgeführt worden. Zusätzlich müsste ein bestimmter Altersdurchschnitt der Patienten als möglich Moderator-Variable ausgeschlossen werden.

\subsubsection{Vergleich komorbider somatischer Störungsbilder}

Um die oben genannte Hypothese in Hinsicht auf somatische Erkrankungen zu prüfen, erfolgte der Vergleich komorbider somatischer Erkrankungen rein benzodiazepinabhängiger und benzodiazepin- und alkoholabhängiger Patienten. 


\subsection{Vergleich der Begleiterkrankungen}

Im Vergleich der somatischen Begleiterkrankungen wie chronischen Schmerzsyndromen, neurologischen Erkrankungen, degenerativen Veränderungen des Bewegungsapparates, Erkrankungen des Herz-Kreislauf-Systems, arteriellem Hypertonus und Stoffwechselerkrankungen, zeigten sich keine signifikanten Unterschiede. zwischen benzodiazepin- und benzodiazepin- und alkoholabhängigen Patienten.

Die Gruppe der zusätzlich alkholabhängigen Patienten zeigte allerdings signifikant mehr im Vorfeld der Entzugstherapie aufgetretene Krampfanfälle $(\mathrm{p}=<0,05)$. Es handelte sich hierbei jedoch um Entzugskrampfanfälle, die durch häufige Alkoholentzüge bedingt waren. Im Falle des reinen Diazepamentzugs sind Krampfanfälle mit 2-3\% eher selten (LAUX und KÖNIG 1985).

\subsection{Vergleich der Leberwerte und der abdomensonographischen Befunde}

Im Vergleich der Leberwerte und der abdomensonographischen Befunde zeigten sich signifikante Gruppenunterschiede.

Im Kollektiv der benzodiazepin- und alkoholabhängigen Patienten zeigte sich deutlich die durch den chronischen Alkoholkonsum ausgelöste Leberzellschädigung. In dieser Gruppe bestand eine Erhöhung aller dokumentierten Leberenzyme. Hochsignifikante Unterschiede zwischen den Gruppen zeigten sich im Grad der Erhöhung der gamma-GT $(p<0,01)$ und der GOT ( $p<0,01)$, die in der Gruppe der zusätzlich alkoholabhängigen Patienten deutlich höhere Werte aufwiesen. Die De-Ritis-Quotienten lagen in dieser Gruppe im Mittel bei 1,24, was auf eine massive Leberzellschädigung hinweist. Trotz numerisch höhrerem Wert, zeigte sich betreffend den De-Ritis-Quotienten aber kein signifikanter Gruppenunterschied.

Passend dazu, zeigte sich auch im Falle der sonographischen Befunde des Abdomens, ein signifikant häufigeres Auftreten $(\mathrm{p}<0,01)$ von pathologischen Veränderungen in der Gruppe der zusätzlich alkoholabhängigen Patienten. So zeigten 63\% der Patienten Veränderungen wie Steatosis hepatis, Hepatomegalie oder Splenomegalie. Der Anteil klinisch relevanter Veränderungen im Sinne von Aszites oder Zeichen einer Leberzirrhose lag dabei mit 3,7\% erstaunlich niedrig. In Hinsicht auf die bekannten lebertoxischen Wirkungen chronischen Alkoholkonsums, waren diese Ergebnisse erwartet. 


\subsection{Vergleich der bildgebenden pathologischen Befunde des Neurokraniums}

Im Vergleich der pathologischen Befunde des Neurokraniums, die durch Auswertung von cCT und cMRT Bildern erfasst wurden, zeigten sich ebenfalls signifikante Gruppenunterschiede.

Im Kollektiv der rein benzodiazepinabhängigen Patienten lagen signifikant mehr mikroangiopathische Veränderungen vor $(\mathrm{p}<0,01)$.

In der Gruppe der zusätzlich alkoholabhängigen Patienten zeigten sich bei 43,5\% der Patienten hirnatrophische Veränderungen. Numerisch waren somit in dieser Gruppe deutlich mehr Patienten von hirnathrophischen Veränderungen betroffen, als in der Gruppe der rein benzodiazepinabhängigen Patienten. Statistisch signifikant war dieser Unterschied aber nicht. Ähnliche Ergebnisse sind in der Literatur hinsichtlich der CT/MRT Untersuchungen alkoholabhängiger Patienten beschrieben. So zeigte sich auch in vorausgehenden Studien eine Assoziation zwischen chronischer Alkoholabhängigkeit und hirnatrophischen Veränderungen (FOX et al. 1976, HILL und MIKHAEL 1979).

\subsection{Zusammenfassung der Vergleiche zwischen den Gruppen}

Zusammenfassend zeigt sich die oben genannte Hypothese in Hinsicht auf somatische Erkrankungen teilweise bestätigt.

Im Vergleich der somatischen Begleiterkrankungen zeigten sich keine signifikanten Unterschiede zwischen rein benzodiazepinabhängigen und benzodiazepin- und alkoholabhängigen Patienten. Die Gruppe der zusätzlich alkholabhängigen Patienten zeigte signifikant mehr im Vorfeld der Entzugstherapie aufgetretene Entzugskrampfanfälle. Weiterhin zeigte das Kollektiv der doppelabhängigen Patienten ein deutlich höheres Ausmaß der Leberzellschädigung und mehr hirnatrophische Veränderungen. Die Gruppe der rein benzodiazepinabhängigen Patienten wies mehr mikroangiopathische Veränderungen des Neurokraniums auf.

\subsubsection{Limitierende Faktoren der Studienergebnisse}

Bei der Interpretation der Ergebnisse müssen verschiedene Limitationen berücksichtigt werden. Der Benzodiazepinentzug erfolgte standardisiert für alle Patienten gleich, um den Einfluss des Medikamentenabbruchs möglichst gering zu halten. Da die Befragung der Patienten jedoch unter noch laufender Medikation im Entzug stattfand, kann nicht ausgeschlossen werden, dass Entzugssymptome die Evaluierung komorbider Störungsbilder 
beeinflusst haben könnten. Möglicherweise führte dies zu erhöhten Diagnosezahlen von begleitenden affektiven und insbesondere Angststörungen. Zusätzlich wurden nicht nur aktuelle, sondern Lebenszeit-Komorbiditäten erfasst, was höhere Diagnosezahlen als in vergleichbaren Studien mitbegründen könnte. Die vorliegenden Daten repräsentieren ein in Kliniken behandeltes Patientenkollektiv und erfassen nicht unbedingt den Durchschnitt benzodiazepinabhängiger Patienten.

Der Mini-Dips, der zur Erfassung komorbider psychiatrischer Störungsbilder verwendet wurde, erfasst keine Achse-II Störungen. Somit konnten Persönlichkeitsstörungen wie z.B. impulsive Störungen, die in Studien mit alkoholabhängigen Patienten häufig beschrieben werden, nicht miterfasst werden.

Auch im Bereich somatischer Erkrankungen könnte die Überlagerung durch Entzugssymptome zu erhöhten Diagnosezahlen geführt haben.

Weiterhin könnten somatische Erkrankungen, die bedingt durch die Methodik nicht miterfasst wurden, zu einer Verschlechterung von Laborwerten, wie z.B. erhöhten Leberwerten, geführt haben. Da ein Großteil der Patienten weitere Medikamente einnahm, können mögliche hepatotoxische Effekte nicht sicher den Benzodiazepinen zugeschrieben werden.

Auch die Ergebnisse der Untersuchung des Neurokraniums sind nur eingeschränkt beurteilbar. Sollten die pathologischen Veränderungen des Neurokraniums in der Gruppe der rein benzodiazepinabhängigen Patienten durch eine langjährige Einnahme von Benzodiazepinen bedingt sein, wären diese Veränderungen auch im Kollektiv der zusätzlich alkoholabhängigen Patienten zu erwarten gewesen, was nicht zutraf. Weiterhin handelt es sich um eine kleine Patientengruppe und es lagen nur in ca 60\% der Fälle CT oder MRT Bilder vor. Auch in der vorliegenden Studie wurden Patienten mit komorbiden medizinischen sowie auch psychiatrischen Erkrankungen beurteilt. Es lässt sich somit nicht ausschließen, dass die Ergebnisse beispielsweise durch vorbestehende neurologische Erkrankungen oder andere Achse-I-Störungen beeinflusst wurden. Zusätzlich nahm ein Großteil der Patienten aufgrund bestehender psychiatrischer komorbider Erkrankungen weitere Medikamente ein, die ebenfalls Auslöser bildgebender Veränderungen sein könnten. Ein weiterer wichtiger Faktor ist das Fehlen alters- und geschlechtsgleicher Kontrollpatienten. 


\subsubsection{Hypothese 2: Benzodiazepinabhängige Patienten haben bei Komorbidität mit einer Alkoholabhängigkeit im Benzodiazepin- entzug ein höheres Ausmaß an kognitiven Defiziten als isoliert benzodiazepinabhängige Patienten}

Ein weiterer großer Anteil der vorliegenden Arbeit befasst sich mit der Untersuchung kognitiver Defizite benzodiazepinabhängiger Patienten. Im Anschluss erfolgte ein Vergleich kognitiver Defizite von benzodiazepinabhängigen und benzodiazepin- und alkoholabhängigen Patienten.

In Bezug auf die möglichen Nebenwirkungen langzeitiger Benzodiazepineinnahme wird wohl am kontroversesten die Beeinflussung kognitiver Funktionen diskutiert. Ziel der vorliegenden Studie war daher die Gewinnung von Daten zu kognitiven Fähigkeiten benzodiazepinabhängiger Patienten. In der Literatur werden Sedierung und die Beeinträchtigung psychomotorischer und kognitiver Fähigkeiten als einige der Hauptprobleme der Akutbehandlung mit Benzodiazepinen gesehen (BARBEE 1993). Weniger klar scheinen jedoch die Auswirkungen langzeitiger Benzodiazepineinnahme auf kognitive Funktionen.

Bisher gibt es wenige Studien, die sich mit kognitiven Fähigkeiten von Patienten im Benzodiazepinentzug befassten. Die Beurteilung kognitiver Parameter, insbesondere im Entzug, ist jedoch bedeutend, da den Patienten bereits zu diesem Zeitpunkt wichtige therapeutische Entscheidungen abverlangt werden und Differentialdiagnosen gestellt werden. Die Kognitions-Screeningtests wurden in der vorliegenden Studie daher erst zu Beginn der zweiten Hälfte des Benzodiazepinentzugs durchgeführt.

\subsubsection{Kognitive Defizite benzodiazepinabhängier Patienten}

Zur Beurteilung von Hirnleistungsstörungen benzodiazepinabhängiger Patienten in der Entzugstherapie verwendeten wir den Uhrentest (Clockdrawing Test nach SHULMAN et al. 1993) und den MMST (Mini Mental Status Test nach FOLSTEIN et al. 1975).

\subsection{Kognitive Defizite im Uhrentest (CDT)}

Es folgt die Beschreibung der Ergebnisse des Uhrentests (CDT nach SHULMAN et al. 1993), der insbesondere die Beurteilung visuokonstruktiver Fähigkeiten erlaubt.

Im gesamten Kollektiv der benzodiazepinabhängigen Patienten zeigten sich hochgradige kognitive Defizite im Uhrentest.

So erreichte nur etwa ein Viertel der Probanden einen Score von eins, der einer korrekt eingezeichneten Uhrzeit entsprach. Mehr als ein Drittel der Probanden erreichte einen bereits 
pathologischen Score von drei, entsprechend einer fehlerhaft eingezeichneten Uhrzeit. Mehr als 10\% der Probanden lagen im Bereich mittelgradiger visuell- räumlicher Desorganisation und über 5\% sogar im Bereich schwergradiger visueller Desorganisation.

\subsection{Kognitive Defizite im Mini Mental Status Test}

Als weiteren Screeningtest zur orientierenden Erfassung kognitiver Defizite verwendeten wir den Mini Mental Status Test (MMST nach FOLSTEIN et al. 1975).

Die Ergebnisse des Mini Mental Status Test deckten sich nicht mit den Ergebnissen der Uhrentests. Die Patienten erreichten im Mittel 28,3 Punkte und somit normale Werte. Das Vorhandensein von Hirnleistungsstörungen wird erst bei Werten unter 25 Punkten angenommen.

\subsection{Vergleich der kognitiven Defizite}

Im Vergleich der Ergebnisse der Uhrentests lagen die Ergebnisse im Kollektiv der rein benzodiazepinabhängigen Patienten mit einem Score von im Mittel 2,6 leichtgradig schlechter als die der zusätzlich alkoholabhängigen Patienten mit einem Score von im Mittel 2,3 Punkten. Statistisch signifikante Unterschiede zwischen den Gruppen der rein benzodiazepinabhängigen Patienten und benzodiazepin- und alkoholabhängigen Patienten ergaben sich aber nicht.

Die Ergebnisse des Mini Mental Status Test lagen mit im Mittel 28,13 Punkten im Falle der rein benzodiazepinabhängigen Patienten und im Mittel 28,47 Punkten im Falle der zusätzlich alkoholabhängigen Patienten gleich. Die oben genannte Hypothese zeigt sich somit nicht bestätigt. Benzodiazepin- und alkoholabhängige Patienten im Benzodiazepinentzug haben nicht mehr kognitive Defizite als rein benzodiazepinabhängige Patienten.

STEWART (2005) sahen ein hohes Risiko kognitiver Beeinflussung insbesondere im Fall von Patienten, die hohe Dosen von Benzodiazepinen erhielten, männlichen Individuen, älteren Patienten und Patienten mit begleitender Abhängigkeit von anderen Drogen oder Alkohol. Dies konnte in der vorliegenden Studie - zumindest in Hinsicht auf Patienten mit einer zusätzlich bestehenden Alkoholabhängigkeit - nicht bestätigt werden.

\subsection{Beurteilung der kognitiven Defizite des gesamten Patientenkollektivs}

Die Resultate zeigen insgesamt eine deutliche Beeinflussung kognitiver Fähigkeiten unter Benzodiazepineinnahme. In der vorliegenden Studie lagen diese Defizite insbesondere im Bereich psychomotorischer/ visuokonstruktiver Fähigkeiten. 
In der Literatur finden sich zahlreiche Hinweise auf die Beeinflussung kognitiver Fähigkeiten durch die kurzzeitige, aber auch durch die langzeitige Einnahme von Benzodiazepinen.

So ist die kurzzeitige, akute Behandlung mit Benzodiazepinen für Nebenwirkungen wie Sedierung, Benommenheit, psychomotorische Verlangsamung, anterograde Amnesien und Schwierigkeiten beim Speichern neuer Informationen bekannt (BARBEE 1993). BUFFETJEROTT und STEWART (2002) stellten jedoch fest, dass viele Patienten Toleranz gegenüber den sedierenden und aufmerksamkeitsbeinträchtigenden Effekten der Benzodiazepine entwickeln.

Dennoch wird auch im Falle von Patienten mit langjähriger Benzodiazepineinnahme über multiple kognitive Dysfunktionen berichtet. So zeigten Metaanalysen von BARKER et al. (2004 a), die sich auf die zwischen 1980 und 2000 publizierten klinischen Studien beziehen, eine Beeinträchtigung von visuell räumlichen Fähigkeiten, Aufmerksamkeit und Konzentration, Problemlösungsvermögen, genereller Intelligenz, psychomotorischer Geschwindigkeit, sensorischer Verarbeitung, verbalem Erinnerungsvermögen, nichtverbalem Erinnerungsvermögen, der Geschwindigkeit der Informationsverarbeitung, motorischen Fähigkeiten, Arbeitsgedächtnis und verbaler Intelligenz. Die Beeinflussung visuell räumlicher Fähigkeiten scheint dabei am spezifischsten mit langzeitiger Benzodiazepineinnahme verbunden (STEWART 2005). GOLOMBOK et al. (1988) führten eine Studie mit 50 langzeitig benzodiazepinabhängigen Patienten durch, in der multiple kognitive Fähigkeiten getestet wurden. Die stärkste Beeinflussung zeigte sich auch hier im Bereich visuell räumlicher Fähigkeiten. GOLOMBOK et al. (1988) verwendeten dabei drei verschiedene Tests zur Beurteilung visueller Parameter: Einen Test zur Messung der motorischen Geschwindigkeit, einen Test zur Messung visuell räumlicher Organisation und einen Test zur Messung räumlicher Orientierung. Die Patienten zeigten in allen drei Tests signifikant schlechtere Ergebnisse als Kontrollen. Auch GOLOMBOK et al. (1988) wiesen hierbei auf die, einer Toleranz unterliegenden sedierenden und Gedächtnis beeinträchtigenden Effekte der akuten Behandlung mit Benzodiazepinen hin. Sie sahen jedoch eine große Diskrepanz zwischen der akuten Behandlung mit Benzodiazepinen und der langfristigen Anwendung von Benzodiazepinen. So scheinen die, das Gedächtnis beeinträchtigenden Effekte kurzzeitiger Therapie, im Falle chronischer Anwendung nicht mehr im Vordergrund zu stehen. Sie sahen die langzeitige Anwendung von Benzodiazepinen vielmehr verknüpft mit einer Beeinträchtigung höherer Hirnfunktionen. In dieser Studie waren dies in erster Linie visuell räumliche Fähigkeiten (GOLOMBOK et al. 1988). 
Eine Studie von TATA et al. (1994) mit 21 langzeitig-benzodiazepinabhängigen Patienten zeigte ebenfalls signifikant beeinträchtigte visuomotorische- und visuell räumliche Fähigkeiten der Patienten im Vergleich mit alters- und IQ-gleichen Kontrollen. Diese Ergebnisse decken sich mit denen der vorliegenden Studie, in der sich die stärkste Beeinflussung ebenfalls im Bereich visuokonstruktiver Fähigkeiten zeigte.

Die Ergebnisse des Mini Mental Status Test als Messparameter globaler kognitiver Funktionen, wiesen in der vorliegenden Studie mit im Mittel 28,3 Punkten, nicht auf starke kognitive Defizite hin. PATERNITI et al. (2001) konnten in einer Studie mit 264 benzodiazepinabhängigen Teilnehmern jedoch zeigen, dass benzodiazepinabhängige Patienten mit im Mittel 27,5 Punkten insgesamt signifikant schlechtere Werte als benzodiazepinfreie Kontrollen erreichten, die bei 28,3 Punkten lagen. Diese Werte befinden sich allerdings nicht im Bereich von Hirnleistungsstörungen, sondern beschreiben eher eine Tendenz. Dennoch zeigte auch die Studie von PATERNITI et al. eine Verschlechterung verschiedener kognitiver Funktionen im Falle chronischer Benzodiazepineinnahme, unabhängig von Alter, Geschlecht, und Bildungsniveau der untersuchten Patienten.

Schwierig scheint insbesondere, dass Patienten im Benzodiazepinentzug die Beeinträchtigung ihrer kognitiven Fähigkeiten kaum wahrzunehmen scheinen und mögliche Defizite erst nach erfolgtem Entzug realisiert werden (GOLOMBOK et al.1988).

Andere Studien fanden keinen signifikanten Zusammenhang zwischen der Beeinflussung kognitiver Fähigkeiten und langfristiger Benzodiazepineinnahme.

LUCKI et al. (1986) verglichen die kognitiven Fähigkeiten von 43 langzeit-benzodiazepinabhängigen Patienten mit komorbider Angststörung, mit einer Kontrollgruppe, die ebenfalls Angststörungen aufwies. Die Testergebnisse der chronisch benzodiazepinabhängigen Patienten unterschieden sich dabei nicht signifikant von denen der benzodiazepinfreien Kontrollgruppe. Auch Mc ANDREWS et al. (2003) testeten in einer Studie mit 25 langzeitbenzodiazepinabhängigen Patienten multiple kognitive Domänen, ohne signifikante Beinträchtigungen feststellen zu können. Sie verwiesen hierbei auf den großen Einfluss psychiatrischer komorbider Erkrankungen auf kognitive Funktionen und schlossen Patienten mit psychiatrischen oder neurologischen Begleiterkrankungen aus.

Traten kognitive Defizite auf, wurden diese oftmals sedierenden Effekten der Benzodiazepine oder geminderter Aufmerksamkeit zugeschrieben. Einige Autoren sahen mögliche kognitive Defizite auch als temporär verknüpft mit Peak-Plasmaleveln an (BUFFET-JEROTT et al. 1998). Diese Thesen lassen sich durch die vorliegende Studie nicht stützen, da die Patienten im Mittel eine ca. 10 Jahre andauernde Benzodiazepineinnahme aufwiesen. Sollten die 
sedierenden Effekte der Benzodiazepine, wie von BUFFET-JERROTT et al. beschreiben, einer Toleranzentwicklung unterliegen, könnten sie somit nicht ausschließlich für die gravierenden kognitiven Defizite im Bereich visuokonstruktiver Fähigkeiten verantwortlich gemacht werden. Auch eine Verknüpfung mit hohen Plasmaleveln scheint in der vorliegenden Studie unwahrscheinlich, da sich die Patienten im Entzug befanden und die Benzodiazepine in Etappen reduziert wurden. Es scheint sich also um einen Trait Marker zu handeln.

Insgesamt zeigen diese Ergebnisse deutlich, dass Patienten, die langzeitig mit Benzodiazepinen behandelt werden, auf mögliche kognitive Defizite hingewiesen werden sollten, die zu schwerwiegenden Problemen im Alltag führen und das soziale und berufliche Leben der Betroffenen stark einschränken können. Obwohl die Verwendung von Benzodiazepinen nur für vier Wochen empfohlen wird, erhalten gerade ältere Patienten mit chronischen Schlafstörungen häufig über lange Zeiträume immer neue Rezepte. Eine dauerhafte positive Wirkung auf die Schlafgewohnheiten der behandelten Patienten ist dabei nicht bewiesen (CURRAN et al. 2003). Die Risiko-Nutzen Analyse der langzeitigen Anwendung von Benzodiazepinen wird intensiv diskutiert. Auf der einen Seite erscheint es wichtig, insbesondere Angstpatienten keine Behandlung vorzuenthalten, wenn sich Alternativen als ineffektiv erweisen. Auf der anderen Seite gilt es, übermäßigen Gebrauch zu verhindern (PATERNITI et al. 2001). In jedem Fall sollte vor der Verschreibung eine genaue psychiatrische Untersuchung erfolgen. Sollte für eine langfristige Therapie mit Benzodiazepinen entschieden werden, müssen, wie auch im Fall jeder anderen Therapie, potentiell negative gegen positive Effekte der Behandlung abgewogen werden.

Unklar ist, wie weit mögliche, beeinträchtigte Fähigkeiten nach erfolgreichem Benzodiazepinentzug wieder remittieren. Einige Studien zeigen eine relativ rasche Erholung kognitiver Fähigkeiten nach erfolgter Entgiftung. So zeigten beispielsweise CURRAN et al. (2003) in einer Studie mit 138 benzodiazepinabhängigen Patienten, dass sich deren kognitive Fähigkeiten 24 bzw. 52 Wochen nach Entzug deutlich besserten. Im Vergleich mit benzodiazepinabhängigen Patienten die keinen Entzug durchliefen, zeigten die Patienten, die einen Entzug durchlaufen hatten, bereits nach 24 Wochen deutlich gebesserte Werte in der Geschwindigkeit der Informationsverarbeitung und nach 52 Wochen gebesserte Werte in den Bereichen des Arbeitsgedächtnisses und visuell-räumlicher Fähigkeiten. Obwohl sich kognitive Dysfunktionen nach dem Entzug verbesserten, konnten Metaanalysen klinischer Studien zeigen, dass Patienten nach langzeitiger Anwendung von Benzodiazepinen bis 6 Monate nach Entzug, nicht die Funktionslevel von Patienten ohne Benzodiazepinkonsum in der Vergangenheit erreichten (BARKER et al. 2004b). Eine von Barker et al. im Anschluss an 
diese Metaanalysen durchgeführte klinische Studie mit 20 benzodiazepinabhängigen Patienten, die seit mindestens 6 Monaten, im Schnitt aber 42 Monaten, benzodiazepinfrei lebten, zeigte auch nach mehr als 6 Monaten noch bestehende kognitive Defizite in den Bereichen verbaler Erinnerung, nichtverbaler Erinnerung und motorischer Funktionen (BARKER et al. 2004c). Barker et al. empfahlen behandelnden Ärzten daher dringend, Patienten vor dem Beginn einer Therapie mit Benzodiazepinen, über die mögliche langzeitige Beeinflussung kognitiver Funktionen zu informieren.

Andere Studien aus dem Bevölkerungsdurchschnitt, die sich insbesondere mit möglichen, bleibenden kognitiven Defiziten älterer benzodiazepinabhängiger Patienten auseinander setzten, ergaben widersprüchliche Daten. So beschreiben VERDOUX et al. (2004) in einem Review eine permanente Schädigung durch langjährige Benzodiazepineinnahme als eher spekulativ. Sie verweisen hierbei auch auf den Faktor, dass sich die meisten Daten auf klinische Studien beziehen, die sich mit besonders schwerwiegenden Fällen beschäftigen und somit nicht auf die Gesamtbevölkerung übertragen werden können (VERDOUX et al. 2004).

\subsection{Limitierende Faktoren}

Verschiedene limitierende Faktoren der vorliegenden Studie führten auch hier zu einer einschränkenden Beurteilbarkeit der erhobenen Daten.

Zunächst handelt es sich mit einem Kollektiv von 60 Patienten bzw. 30 Patienten pro Gruppe, um eine relativ geringe Gruppengröße. Erschwerend kommt hinzu, dass keine Kontrollgruppe getestet wurde. Ein Problem der Wertung der gemessenen kognitiven Defizite der Patienten liegt darin, dass der Entzug zum Zeitpunkt der Befragung erst zur Hälfte abgeschlossen war. Eine Überlagerung durch Entzugssymptome, die zu einer Verschlechterung der Ergebnisse geführt haben könnte, lässt sich somit letztendlich nicht ausschließen. Durch eine leitliniengerechte, langsame Reduktionstherapie wurde versucht, starke Entzugssymptome zu vermeiden. Sollten die kognitiven Defizite dennoch zu einem gravierenden Anteil durch noch bestehende Entzugssymptome ausgelöst worden sein, wäre eine Beeinträchtigung der Ergebnisse aller Test zu erwarten gewesen. Einschränkungen zeigten sich jedoch nur im Bereich visuokonstruktiver Fähigkeiten, gemessen durch den Clock Drawing Test.

Auch LUCKI et al. (1986) testeten in ihrer Studie die kognitiven Fähigkeiten von 17 der 43 benzodiazepinabhängigen Patienten nach der Basisuntersuchung erneut im Entzug. Hierbei zeigte lediglich einer der verwendeten kognitiven Tests eine leichte Verbesserung. Signifikante Verschlechterungen der Testergebnisse, ausgelöst nur durch bestehende Entzugssymptome, erscheinen daher eher unwahrscheinlich. 
In der vorliegenden Studie ist die Beurteilbarkeit kognitiver Defizite auch über den Entzug hinaus nicht gegeben. Bei zukünftigen Studien sollten Nachuntersuchungen betreffend persistierenden kognitiven Defiziten erfolgen.

Ein weiteres Problem der Beurteilung kognitiver Fähigkeiten liegt darin, dass die langjährige Anwendung von Benzodiazepinen zu einem großen Anteil Patienten mit chronischen Angsterkrankungen betrifft. Auch in der vorliegenden Studie erfüllten 78\% der Patienten die Kriterien einer begleitenden Angststörung. Auch Angsterkrankungen können zu einer Minderung von Aufmerksamkeit und Konzentration führen, dennoch wäre auch in diesem Falle eine Verschlechterung aller kognitiven Fähigkeiten und nicht nur der visuokonstruktiven Fähigkeiten zu erwarten gewesen. In Übereinstimmung mit anderen Studien handelt es sich wahrscheinlich um einen substanzbezogenen Effekt.

Wir versuchten, Patienten mit klinisch relevanten hirnorganischen Vorschäden oder Demenzen aus der Studie auszuschließen. Zur Überprüfung eines Zusammenhanges zwischen pathologischen Veränderungen des Neurokraniums und kognitiven Defiziten wurden entspechende Korrelationsrechnungen durchgeführt. Signifikante Zusammenhänge ergaben sich hierbei nicht. Ebenso wurden Patienten mit einer weiteren Abhängigkeit, von illegalen Substanzen oder psychotropen Medikamenten, aus der Studie ausgeschlossen um sicherzustellen, dass mögliche Medikamenteneffekte ausschließlich den Benzodiazepinen zuzuschreiben sind. Mit 40,7\% nahm ein großer Anteil der Patienten jedoch zusätzlich Antidepressiva ein, in ca 60\% der Fälle waren dies Tri/Tetrazyklische Antidepressiva. Dies könnte wiederum zu einer medikamentösen Beeinflussung durch deren anticholinerge Wirkung geführt haben. Die statistische Analyse der vorliegenden Studie zeigte allerdings keine Unterschiede betreffend kognitiven Fähigkeiten zwischen Patienten unter antidepressiver Medikation und Teilnehmern ohne weitere Medikation. Auch GOLOMBOK et al. (1988) konnten in ihrer Studie eine Beeinflussung psychomotorischer/ visueller Fähigkeiten benzodiazepinabhängiger Patienten - zumindest im Fall trizyklischer Antidepressiva weitestgehend ausschließen.

Weitere psychotrope Medikamente, die begleitend eingenommen wurden, waren in 31,7\% der Fälle Antikonvulsiva und in 20,3\% der Fälle Neuroleptika. Auch im Falle dieser Patienten zeigte die statistische Analyse keine schlechteren Ergebnisse betreffend kognitiven Fähigkeiten zwischen Patienten unter Medikation und Teilnehmern ohne weitere Medikation. Es handelt sich bei der vorliegenden Arbeit um eine klinische Studie, die nur Patienten in psychiatrischer Behandlung berücksichtigt. Die Ergebnisse sind daher schwierig auf die Gesamtbevölkerung zu beziehen, da möglicherweise besonders schwerkranke Patienten 
getestet wurden. In folgenden Studien wäre daher eine Testung kognitiver Fähigkeiten ambulant behandelter Patienten wünschenswert.

\subsubsection{Hypothese 3: Benzodiazepinabhängige Patienten unterscheiden sich von benzodiazepin- und alkoholabhängigen Patienten im Benzodiazepinentzug hinsichtlich des Schweregrades der Abhängigkeit}

Um den Schweregrad der Abhängigkeit in verschiedenen potentiellen Problembereichen erfassen zu können, verwendeten wir den EuropASI (nach GSELLHOFER et al. 1999). Als weiterer Parameter zur Beurteilung des Schweregrades der Abhängigkeit wurden die Entzugsstartdosen verwendet, da sie die Dosis der im Vorfeld eingenommenen Benzodiazepinmenge wiedergeben. Zusätzlich verwendeten wir die PWC (nach RICKELS et al. 1990), um die Schwere der Entzugssymptomatik messen zu können.

Nach Auswertung der Daten erfolgte ein Vergleich des Schweregrades der Abhängigkeit von rein benzodiazepinabhängigen und benzodiazepin- und alkoholabhängigen Patienten.

\subsubsection{Schweregrad der Abhängigkeit des gesamten Patientenkollektivs}

Im gesamten Kollektiv zeigte sich im EuropASI eine Ausprägung von Problemen in den Bereichen körperlicher Zustand, psychische Situation, ökonomischer Status und familiäre Situation. Diese Ergebnisse passen gut zu den im Vorfeld erhobenen Befunden.

Der hohe Anteil komorbider somatischer Begleiterkrankungen (siehe oben) spiegelt sich in hohen Scorewerten und Schweregraden im Bereich körperlicher Zustand des ASI wieder.

Der hohe Anteil komorbider psychiatrischer Störungsbilder führte ebenfalls zu entsprechenden Werten des Bereichs psychischer Zustand im ASI. Die soziodemographischen Ergebnisse, die einen hohen Anteil an arbeitslosen oder bereits berenteten Patienten zeigten, entsprachen der hohen Ausprägung von Problemen im Bereich des ökonomischen Status. Ebenso führte der hohe Anteil geschiedener oder lediger Patienten zu einer hohen Ausprägung von Problemen im Bereich der familiären Situation.

Im Problembereich Drogengebrauch, der verschiedene Substanzabhängigkeiten erfasst und zur Abschätzung der Schwere der Benzodiazepinabhängigkeit von besonderem Interesse ist, zeigten sich im gesamten Kollektiv eher niedrige Scores. Da es sich aus klinischer Sicht um teilweise schwer benzodiazepinabhängige Patienten handelte, passten diese Ergebnisse nicht zum Patientenkollektiv. Aus unserer Sicht liegt dies in einer eingeschränkten Beurteilbarkeit des ASI zur Einschätzung benzodiazepinabhängiger Patienten begründet. Einen wesentlichen 
Parameter beim Berechnen der Schwere der Abhängigkeit stellen beim ASI die finanziellen Ausgaben für den Suchtstoff dar. Benzodiazepine sind generell rezeptpflichtig, was zu sehr geringen finanziellen Ausgaben führt, wenn die Benzodiazepine nicht auf dem Schwarzmarkt erworben werden. Dies führte in der vorliegenden Studie zu durchschnittlich eher geringen Scores, auch im Falle teilweise schwerer Abhängigkeiten. Der ASI erscheint uns daher als Instrument zur Abschätzung einer Benzodiazepinabhängigkeit nur eingeschränkt geeignet. Deutlicher zeigten die hohen Werte der Schweregrade des ASI das Ausmaß der Abhängigkeit. Weiterhin verwendeten wir die Entzugsstartdosen zur Beurteilung des Schweregrades der Benzodiazepinabhängigkeit. Diese lagen im gesamten Kollektiv Mittel bei 22,6 mg Diazepam pro Tag. Die PWC (nach RICKELS et al. 1990) wurde verwendet, um die Schwere der Entzugssymptomatik messen zu können. An Entzugssymptomen zeigten sich in erster Linie Ängste und depressive Symptome. Auch in der Literatur werden im psychischen Bereich an Entzugserscheinungen vor allem Ängste und depressive Symptome vorbeschrieben (POSER W et al. 2006, LADER 2011). Differentialdiagnostisch käme als Ursache der beschriebenen Symptome auch um ein Wiederauftreten der Grunderkrankung in Frage, da ein großer Teil der Patienten bereits im Vorfeld unter Angststörungen oder Depressionen litt.

An vegetativen Entzugserscheinungen zeigten sich insbesondere Schlafstörungen. Auch in der Literatur wird Schlaflosigkeit als eines der häufigsten Symptome des Benzodiazepinentzuges angegeben (POSER W et al. 2006, LADER 2011). So reichen wenige Tage bis Wochen der kontinuierlichen Einnahme aus, um in der Absetzphase leichte Einschlafstörungen zu verursachen. Diese Einschlafstörung wird auch als Rebound-Insomnie bezeichnet, was andeutet, dass dem verbesserten Einschlafen unter Benzodiazepinen eine Verschlechterung in der Entzugsphase folgt (POSER W und POSER S 1996). Weitere häufige physische Symptome waren insbesondere Ermüdung, Ruhelosigkeit, Konzentrationsschwerigkeiten, Zittern und starkes Schwitzen. Auch diese Daten zeigten sich analog zu vorausgehenden Studien (LAUX et al. 1985, RICKELS et al. 2008). Auch perzeptuelle Störungen wurden von einem Teil der Patienten beschrieben. Diese sind typisch für den Entzug von Benzodiazepinen und äußern sich z.B. durch Hyperakusis, Lichtscheu oder eine verstärkte Wahrnehmung von Gerüchen. Die Entzugssymptome, als Parameter des Schweregrades der Abhängigkeit, zeigten gemessen an den Rickels-Scores eine eher mittelschwere Ausprägung. Das gesamte Kollektiv lag im Mittel bei einem Gesamtscore von 18,7 Punkten, bei einem maximal erreichbaren Scorewert von 60 Punkten. 


\subsubsection{Vergleich des Schweregrades der Abhängigkeit}

Im EuropASI zeigte sich in der Gruppe der rein benzodiazepinabhängigen Patienten eine etwas höhere Ausprägung körperlicher Probleme. Diese Ergebnisse passen zu dem hohen Anteil von Patienten mit somatoformen Störungen in dieser Gruppe. Da die Ergebnisse des ASI auf Patientenaussagen gründen, scheinen höhere Scores im Bereich "körperlicher Zustand“, im Falle von Patienten mit somatoformen Störungen, verständlich. Signifikante Gruppenunterschiede bestanden im Bereich körperlicher Probleme aber nicht.

Die Gruppe der zusätzlich alkoholabhängigen Patienten zeigte eine etwas höhere Ausprägung von Problemen, im Bereich des ökonomischen Status und der familiären Situation. Dieses Ergebnis deckt sich mit vorausgehenden Studien, die eine deutliche Einschränkung sozialer Funktionen, insbesondere im Fall alkoholabhängiger Patienten, zeigten (SCHNEIDER et al. 2001). Auch die Schweregrade der Abhängigkeit lagen in der Gruppe der zusätzlich alkoholabhängigen Patienten etwas höher als in der Gruppe der rein benzodiazepinabhängigen. Statistisch signifikante Unterschiede zwischen den Gruppen lagen jedoch in keinem der Bereiche vor.

Auch in der Höhe der Entzugsstartdosen ergaben sich keine signifikanten Gruppenunterschiede. Die Form oder Schwere der Entzugssymptome, gemessen durch die PWC (nach RICKELS et al. 1990), zeigte ebenfalls keine signifikanten Unterschiede zwischen den Gruppen.

Somit zeigte sich oben genannte Hypothese nicht bestätigt. In der vorliegenden Studie ergab sich kein Unterschied im Schweregrad der Abhängigkeit isoliert benzodiazepinabhängiger und doppelabhängiger Patienten. Wobei jedoch erneut auf die eingeschränkte Eignung der Erhebungsinstrumente hingewiesen werden muss.

\subsubsection{Hypothese 3.1: Ein höherer Schweregrad der Abhängigkeit beider Patientengruppen korreliert mit dem Vorhandensein von psychiatrischen und somatischen Komorbiditäten}

Im Anschluss wurde überprüft, ob ein höherer Schweregrad der Abhängigkeit mit psychiatrischen und auch somatischen komorbiden Erkrankungen in Verbindung steht.

Im Bereich psychiatrischer komorbider Störungsbilder stellten wir eine signifikante negative Korrelation zwischen dem Vorliegen von posttraumatischen Belastungsstörungen und hohen Schweregraden von Abhängigkeit, in der Gruppe der rein benzodiazepinabhängigen Patienten, fest. Die Patienten mit PTBS scheinen somit eher von einer Niedrigdosis- 
abhängigkeit betroffen. Diese scheint sich in einem weniger ausgeprägten Suchtverhalten auszudrücken.

Im Bereich somatischer komorbider Störungsbilder ergaben sich in der Gruppe der zusätzlich alkoholabhängigen Patienten, signifikante Korrelationen zwischen einem hohen Schweregrad von Abhängigkeit und pathologisch veränderten Leberwerten, pathologischen sonographischen-Befunden des Abdomens und hirnatrophischen Veränderungen. Hier zeigten sich somit die Auswirkungen schwerer Abhängigkeit auf somatische Befunde. In diesem Fall in Form toxischer Leberschädigungen und hirnatrophischer Veränderungen durch starken Alkoholkonsum.

In der Gruppe der isoliert benzodiazepinabhängigen Patienten ergab sich eine negative Korrelationen zwischen einem hohen Schweregrade von Abhängigkeit und somatischen Erkrankungen, wie Herz-Kreislauf-Erkrankungen oder degenerativen Erkrankungen des Bewegungsapparates. Möglicherweise könnte dieser Faktor darauf hinweisen, dass Patienten, die Benzodiazepine aufgrund von rein somatischen Erkrankungen einnehmen, eher leichtgradigere Formen der Abhängigkeit aufweisen als Patienten, die beispielsweise unter komorbiden psychischen Störungen leiden. Die oben genannte Hypothese konnte somit bestätigt werden.

\subsubsection{Hypothese 3.2: Ein höherer Schweregrad der Abhängigkeit korreliert mit einem höheren Ausmaß an kognitiven Defiziten im Benzodiazepinentzug}

Ein statistisch signifikanter Zusammenhang zwischen besonders schweren Formen der Abhängigkeit und der Ausprägung kognitiver Defizite konnte in der vorliegenden Studie nicht festgestellt werden. Die Hypothese zeigt sich somit nicht bestätigt.

\subsubsection{Hypothese 3.3: Ein höherer Schweregrad der Abhängigkeit korreliert mit der Anzahl bisheriger Entgiftungsbehandlungen}

Der Schweregrad der Abhängigkeit stand in der vorliegenden Studie in keinem Zusammenhang mit der Anzahl im Vorfeld erfolgter Entgiftungsbehandlungen. Auch diese Hypothese konnte nicht bestätigt werden. 


\subsubsection{Hypothese 3.4: Das Vorhandensein psychiatrischer Komorbiditäten korreliert mit der Anzahl bisheriger Entgiftungsbehandlungen}

In der Gruppe der rein benzodiazepinabhängigen Patienten zeigte sich hierbei ein signifikanter Zusammenhang zwischen der Anzahl bisheriger Entgiftungsbehandlungen und der Häufigkeit des Auftretens affektiver Störungsbilder. Patienten, mit mehr im Vorfeld stattgehabten Entgiftungen, zeigten signifikant weniger affektive Störungen. Dies könnte auf eine mangelnde Motivation zur Wahrnehmung spezifischer Therapien, insbesondere dieser Patientengruppe, hinweisen. Möglicherweise steht auch in der hausärztlichen Versorgung die Behandlung der bestehenden affektiven Erkrankung mehr im Vordergrund, als die Behandlung der Suchterkrankung, oder weist auf eine Abneigung zur Entgiftung bei depressiven Patienten hin.

SCHWEIZER et al. (1990) konnten zeigen, dass benzodiazepinabhängige Patienten mit zum Entzugszeitpunkt bestehenden Depressionen oder Angststörungen, auch deutlich schwerere Entzugssymptome und dauerhaft niedrigere Abstinenzraten aufwiesen. Die oben genannte Hypothese zeigt sich bestätigt.

\subsubsection{Hypothese 3.5: Ein höherer ASI-Score des Problembereiches „körperlicher Zustand“ korreliert mit erhöhten Laborwerten, pathologischen Abdomensonographie-Befunden, cCT-, c-MRT-Befunden, EEG-Befunden oder anderen somatischen Begleiterkrankungen}

Wir setzten hierbei alle erhobenen somatischen Befunde mit den Scores und Schweregraden des ASI in Verbindung, wobei sich lediglich eine signifikant negative Korrelation zwischen hohen Parametern von Abhängigkeit und chronischen Schmerzsyndromen, degenerativen Veränderungen des Bewegungsapparates und Herz-Kreislauf Erkrankungen zeigte. Auf Grund der Vielzahl von gerechneten Korrelationen, wird jedoch eine Inflation des Alphafehlerniveaus erwartet. Wendet man daher die Bonferronikorrektur des Alphafehlerniveaus an, sind die signifikanten Ergebnisse eher als Zufallsbefunde zu interpretieren. Wir sehen die oben genannte Hypothese daher als eher nicht bestätig. 


\section{Zusammenfassung}

In der vorliegenden Studie wurden Daten von 30 Patienten mit isolierter Benzodiazepinabhängigkeit und 30 Patienten mit Benzodiazepin- und zusätzlicher Alkoholabhängigkeit erhoben und analysiert. Retrospektiv erfolgte zusätzlich eine Auswertung der Krankenakten.

Analog zu vorausgehenden Studien zeigte sich, dass benzodiazepinabhängige Patienten, die sich in spezialisierten Kliniken im Benzodiazepinentzug befinden, ein hohes Ausmaß an lebenslang oder aktuell bestehenden psychiatrischen Begleiterkrankungen aufweisen. In 85\% der Fälle zeigten die Patienten affektive Störungen, davon machten 80\% monopolare Depressionen aus. Weiterhin wiesen 78\% der Patienten Angsterkrankungen auf, vorwiegend Panikstörungen. Somatoforme Störungen zeigten 28,3\% der Patienten, hierbei wurden insbesondere Somatisierungsstörungen beschrieben.

Auffallend war im Vergleich der beiden Patientengruppen jedoch ein signifikant höheres Ausmaß an komorbiden somatoformen Störungen in der Gruppe der isoliert benzodiazepinabhängigen Patienten. So scheint Benzodiazepinabhängigkeit, stärker als andere Formen des Substanzmissbrauchs, mit somatoformen Störungen verbunden. Es handelte sich hierbei insbesondere um Niedrigdosisabhängigkeiten.

Insgesamt zeigt der hohe Anteil psychiatrischer Komorbiditäten benzodiazepinabhängiger Patienten, wie wichtig es ist diese Komorbiditäten zu erkennen, da sie großen Einfluss auf das Outcome der Patienten haben können. Spezifische Behandlungen der verschiedenen Krankheitsbilder scheinen daher notwendig - in der Prävention des Auftretens von Substanzmissbrauch und in der Rückfallprophylaxe.

In der vorliegenden Studie konnte ebenfalls gezeigt werden, dass Benzodiazepinabhängigkeit in einem engen Zusammenhang mit somatischen Diagnosen steht. So wiesen $48 \%$ der Patienten Erkrankungen des Bewegungsapparates auf und 45\% chronische Schmerzsyndrome. Einen weiteren großen Anteil machten mit 33\% der Fälle kardiovaskuläre Erkrankungen und mit 23\% der Fälle neurologische Erkrankungen aus. Signifikante Gruppenunterschiede bestanden hierbei nicht.

Bemerkenswert war in der vorliegenden Studie insbesondere das hohe Ausmaß kognitiver Defizite. Diese lagen insbesondere im Bereich visuokonstruktiver Fähigkeiten, gemessen durch den Clockdrawing Test (CDT nach SHULMAN et al. 1993). So erreichten im gesamten Kollektiv nur etwa ein Viertel der Patienten einen Score von 1 entsprechend einer korrekten Darstellung der Uhr. Mehr als ein Drittel der Patienten wies einen bereits pathologischen 
Score von 3 auf, entsprechend einer fehlerhaften eingezeichneten Uhrzeit. Über 10\% der Patienten lagen bereits im Bereich mittelgradiger visuell-räumlicher Desorganisation und 5,1\% im Bereich schwergradiger visueller Desorganisation. Die Ergebnisse des Mini Mental Status Test deckten sich mit diesen Ergebnissen nicht. Die Patienten erreichten im Mittel 28,3 Punkte. Von Hirnleistungsstörungen wird erst ausgegangen, wenn weniger als 24 Punkte erreicht werden. Dennoch zeigen diese Ergebnisse eine deutliche Beeinflussung kognitiver Fähigkeiten unter Benzodiazepineinnahme. Signifikante Gruppenunterschiede bestanden hierbei nicht.

Grundsätzlich machen diese Ergebnisse die Notwendigkeit deutlich, Patienten, die langzeitig mit Benzodiazepinen behandelt werden, auf mögliche kognitive Defizite hinzuweisen. Die Einschränkung insbesondere visuokonstruktiver Fähigkeiten kann zu schwerwiegenden Problemen im Alltag führen. Weiterhin kann die Beeinflussung kognitiver Fähigkeiten das soziale und berufliche Leben der Betroffenen stark einschränken. In zukünftigen Studien sollten kognitive Defizite benzodiazepinabhängiger Patienten weiter untersucht werden.

In Bezug auf hepatotoxische Effekte des Substanzmissbrauchs zeigten sich in der vorliegenden Studie bereits massive Leberschädigungen im Kollektiv der zusätzlich alkoholabhängigen Patienten. Es zeigte sich hierbei ein signifikanter Zusammenhang zwischen der Schwere der Abhängigkeit und bestehenden Leberschädigungen. Diese Befunde wurden weitestgehend als erwartet gewertet.

Dennoch bestanden auch im Kollektiv der isoliert benzodiazepinabhängigen Patienten Hinweise auf mögliche toxische Leberzellschädigungen. So zeigte sich eine leichtgradige Erhöhung der gamma-GT sowie ein auffallend hoher De-Ritis-Quotient von im Mittel 1,04. Auch diese Ergebnisse sollten durch zukünftige Studien verifiziert werden.

Unterschiede im Schweregrad der Abhängigkeit isoliert benzodiazepinabhängiger und zusätzlich alkoholabhängiger Patienten ergaben sich in der Studie nicht.

Insgesamt weist die vorliegende Studie deutlich auf massive mögliche Nebenwirkungen langfristiger Benzodiazepineinnahme hin. Diese liegen insbesondere im Bereich kognitiver Fähigkeiten. Sie zeigt die Dringlichkeit einer genauen Indikationsstellung, bevor langfristige Benzodiazepingaben geplant werden. Die aktuell praktizierten Strategien in der Therapie sollten daher überprüft und entsprechend verändert werden. 


\section{Anhang}

\subsection{Fragebogen Mini Mental Status Test (MMST)}

\section{Mini-Mental Status Test (MMST)}

Name

Datum

Score

\section{A. Orientierung}

Zeit

(z. B. Welchen Tag haben wir heute?)

1. Jahr

2. Jahreszeit

3. Datum

4. Wochentag

Ort

(z. B. Wo sind wir?)

5. Monat

6. Land/Staat

7. Bundesland

8. Stadt/Ortschaft

9. Klinik/Praxis/Altersheim

10. Stockwerk

Summe (max. 10):

\section{B. Merkfähigkeit}

Der Untersucher nennt folgende drei Gegenstände und fordert den Patienten auf, die Begriffe zu wiederholen (1 Punkt für jede richtige Antwort) Der Untersucher wiederholt die Wörter so lange, bis der Patient alle drei

1. $>$ Auto $<$

2. $>$ Blume< gelernt hat (höchstens 6 Wiederholungen).

3. $>$ Kerze<

\section{Aufmerksamkeit und Rechenfähigkeit}

Von 100 an sind jeweils 7 abzuziehen. Falls ein Rechenfehler gemacht wird und die darauf folgenden Ergebnisse „verschoben" sind, so wird nur ein Fehler gegeben.

ODER

Falls der Patient die Aufgabe nicht durchführen kann oder will, „RADIO“ rückwärts buchstabieren lassen: O-I-D-A-R

$\begin{array}{ll}1 . & \mathrm{O} \\ 2 . & \mathrm{I} \\ 3 . & \mathrm{D} \\ 4 . & \mathrm{A}\end{array}$

\section{Erinnerungsfähigkeit}

Der Untersucher fragt nach den drei zuvor genannten Wörtern.

$R$

\section{E. Sprache}

Der Untersucher zeigt zwei Gegenstände und fordert den Patienten auf, sie zu benennen.

Der Untersucher fordert den Patienten auf, nachzusprechen

Der Untersucher läßt den Patienten folgendes Kommando befolgen.

Der Untersucher bittet den Patienten,

Der Untersucher dreht das Blatt um und fordert den Patienten auf,

1. Armbanduhr

2. Bleistift

3. "Sie leiht ihm kein Geld mehr"

4. „Nehmen Sie dieses Blatt in die rechte Hand"

5. "Falten Sie es in der Mitte"

6. "Legen Sie es auf den Boden“

7. die Anweisung auf der Rückseite zu befolgen

8. einen vollständigen Satz zu schreiben (Rückseite).

9. Nachzeichnen (Rückseite)

Summe (max. 9): 


\section{Bitte schließen Sie die Augen!}

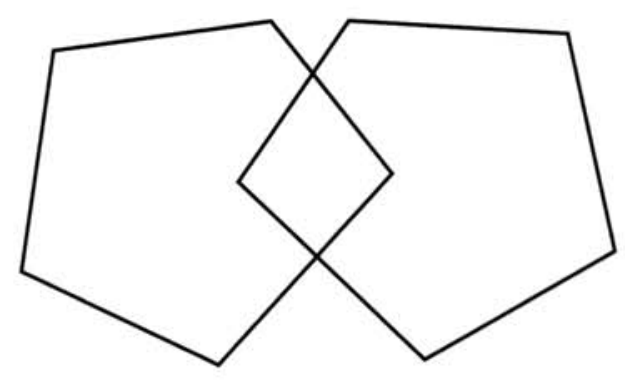




\subsection{Uhrentest (Clockdrawing Test, CDT)}
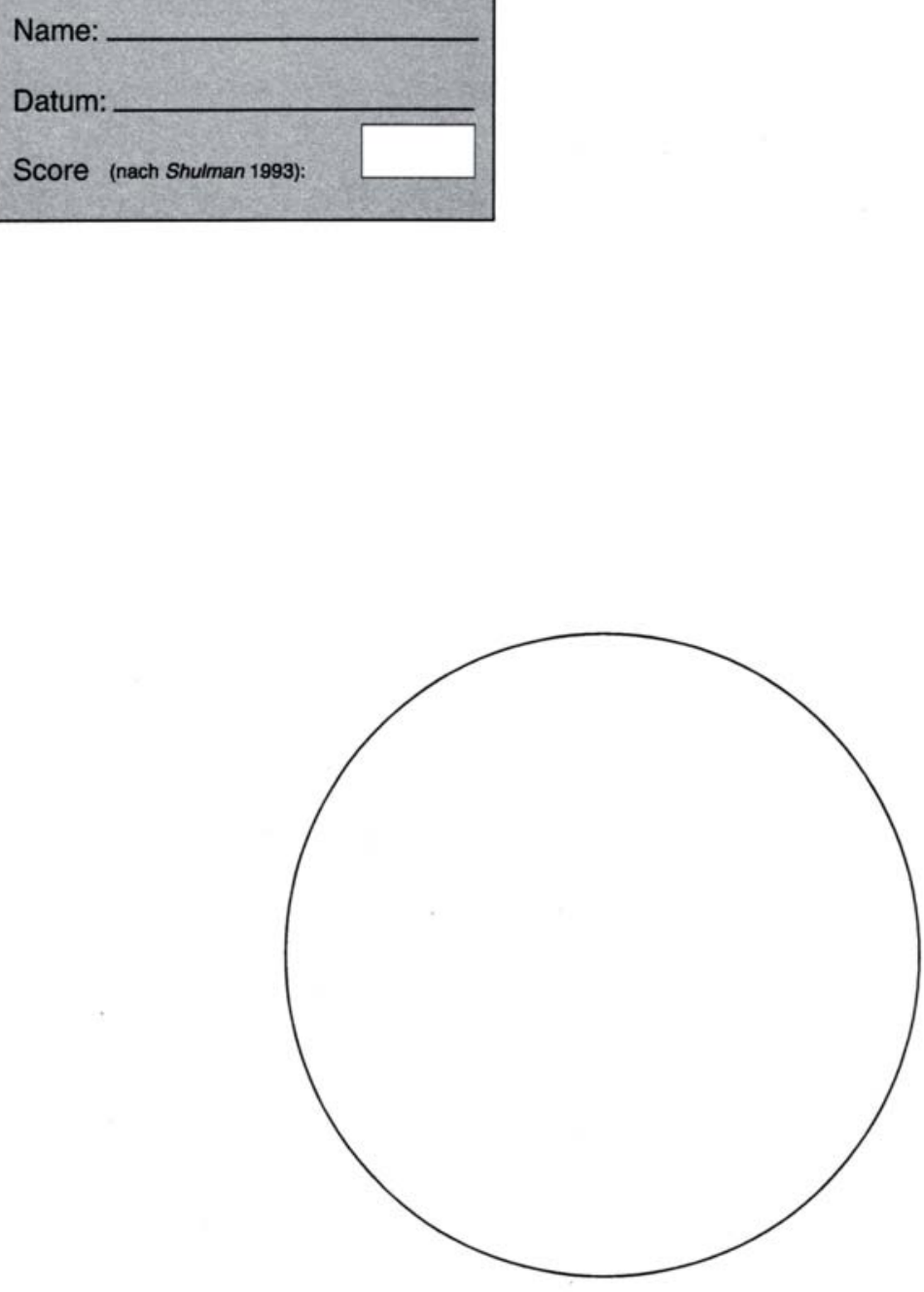


\section{Literaturverzeichnis}

Allison C, Pratt JA (2003): Neuroadaptive processes in GABAergic and glutamatergic systems in benzodiazepine dependence. Pharmacol Ther $\underline{98}$, 171-195

APA (American Psychiatric Association): Diagnostic and statistical manual of mental disorders, 4th edition; Washington D.C. 1994

Barbee JG (1993): Memory, benzodiazepines, and anxiety: integration of theoretical and clinical perspectives. J Clin Psychiatry 54(10 suppl), 86-97

Barker MJ, Greenwood KM, Jackson M, Crowe SF (2004a): Cognitive effects of long-term benzodiazepine use: a meta-analysis. CNS Drugs 18(1), 37-48

Barker MJ, Greenwood KM, Jackson M, Crowe SF (2004b): Persistence of cognitive effects after withdrawal from long- term benzodiazepine use: a meta-analysis. Arch Clin Neuropsychol $\underline{19}$, 437-454

Barker MJ, Greenwood KM, Jackson M, Crowe SF (2004c): An evaluation of persisting cognitive effects after withdrawal from long- term benzodiazepine use. J Int Neuropsychol Soc 11(3), 281-289

Barlow DH (1997): Anxiety disorders comorbid substance abuse, and benzodiazepine discontinuation. NIDA Res Monogr 172, 33-51

Brunette MF, Noordsy DL, Xie H, Drake RE (2003): Benzodiazepine use and abuse among patients with severe mental illness and co-occurring substance use disorders. Psychiatr Serv 54(10), 1395-1401

Buffet-Jerott SE, Stewart SH (2002): Cognitive and sedative effects of benzodiazepine use. Curr Pharm Des 8(1), 45-58

Buffet-Jerott SE, Stewart SH, Bird S (1998): An examination of differences in the time course of oxazepam's effects on implicit versus explicit memory. J Psychopharmacol 12(4), 338-347

Busto UE, Romach MK, Sellers EM (1996). Multiple drug use and psychiatric comorbidity in patients admitted to the hospital with severe benzodiazepine dependence. J Clin Psychopharmacol 16(1), 51-57

Busto UE, Bremmer KE, Knight K, terBrugge K, Sellers EM (2000): Long-term benzodiazepine therapy does not result in brain abnormalities. J Clin Psychopharmacol 20(1), 2-6

Charney DA, Paraherakis AM, Gill KJ (2000): The treatment of sedative-hypnotic dependence: evaluating clinical predictors of outcome. J Clin Psychiatry $\underline{61(3)}$, 190-195

Chatterjee S, Chatterjee J, Chaundhuri K, Shyamal B. Maiti C (1997): Hepatotoxicity of diazepam. Structural and trace metal studies in rat. Biol Trace Elem Res 57(3), 239-250 
Curran HV, Collins R, Fletcher S, Kee SCY, Woods B, Iliffe S (2003): Older adults and withdrawal from benzodiazepines hypnotics in general practice: effects on cognitive function, sleep, mood and quality of life. Psychol Med 33(7), 1223-1237

De Wit H, Doty P (1994): Preference for ethanol and diazepam in light and moderate social drinkers: a within-subjects study. Psychopharmacology (Berl) 115(4), 529-538

Driessen M, Schulte S, Luedecke C, Schaefer I, Sutmann F, Ohlmeier M, Kemper U, Koesters G, Chodzinski C, Schneider U, Broese T, Dette C, Havemann-Reinecke U (2008): Trauma and PTSD in patients with alcohol, drug, or dual dependence: a multi-center study. Alcohol Clin Exp Res 32(3), 481-488

Folstein MF, Folstein SE, McHugh PR (1975): "Mini Mental State“: A practical method for grading the cognitive state of patients for the clinician. J Psychiatr Res $\underline{12(3)}$, 189-198

Fox JH, Ramsey RG, Huckman MS, Proske AE (1976): Cerebral ventricular enlargement: chronic alcoholics examined by computerized tomography. JAMA-J Am Med Assoc 236(4), 365-368

Funderburk FR, Griffiths RR, McLeod DR, Bigelow GE, Mackenzie A, Liebson IA, NemethCoslett RE (1988): Relative abuse liability of lorazepam and diazepam: an evaluation in „recreational“ drug users. Drug Alcohol Depen 22(3), 215-222

Glaeske G: Medikamente - Psychotrope und andere Arzneimittel mit Missbrauchs- und Abhängigkeitspotenzial; in: Jahrbuch Sucht der Deutschen Hauptstelle für Suchtfragen; hrsg.v. Gaßmann R, Kaldewei D, Lindemann F, Merfert-Diete C; Neuland, Geesthacht 2010, 69-95

Golombok S, Moodley P, Lader M (1988): Cognitive impairment in long-term benzodiazepine users. Psychol Med 18, 365-374

Gonzales J, Stern T, Emmerich A, Rauch S (1992): Recognition and management of benzodiazepine dependence. Am Fam Physician 45(5), 2269-2276

Grant BF, Stinson FS, Dawson DA, Chou P, Dufour MC, Compton W, Pickering RP, Kaplan K (2004): Prevalence and co-occurrence of substance use disorders and independent mood and axiety dsorders. Results from the National Epidemiologic Survey on Alcohol and Related Conditions. Arch Gen Psychiatry $\underline{61(8)}$, 807-816

Gsellhofer B, Küfner H, Vogt M, Weiler D: European Addiction Severity Index, EuropASI, Schneider, Hohengehren 1999

Gürtler K, Szecsey A, Stöhr H (1997): Psychometrische Demenzdiagnostik in der klinischen Praxis der Gerontopsychiatrie. Z Gerontol Geriatr 31(4), 281-285

Hajak G, Müller WE, Wittchen HU, Pittrow D, Kirch W (2003): Abuse and dependence potential fot the non-benzodiazepine hypnotics zolpidem and zopiclone: a review of case reports and epidemiological data. Addiction 98(10), 1371-1378 
Hasin D, Katz H (2007): Somatoform and substance use disorders. Psychosom Med 69 (9), 870-875

Havemann-Reinecke U: Die Bedeutung zentraler dopaminerger Neurone für die Entwicklung von Abhängigkeit am Beispiel der Opioide. Shaker, Göttingen 1998

Hemmelgarn B, Suissa S, Huang A, Boivin JF, Pinard G (1997): Benzodiazepine use and the risk of motor vehicle crash in the elderly. JAMA-J Am Med Assoc 278(1), 27-31

Herold G: Innere Medizin. Eine Vorlesungsorientierte Dastellung; hrsg. v. Gerald Herold, Köln 2008

Hill SY, Mikhael M (1979): Computerized transaxial tomography (CTT) and neuropsychological evaluations in chronic alcoholics and heroin addicts.

Am J Psychiatry 136(4B), 598-602

Hoffman RS, Wipfler FG, Maddaloni MS (1991): Has the New York State triplicate benzodiazepine prescription influenced sedative hypnotic overdose?

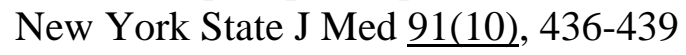

Hollister LE, Motzenbecker FP, Degan RO (1961): Withdrawal reactions from chlordiazepoxid (“Librium”). Psychopharmacologia 2, 63-68

Holzbach R, Martens M, Kalke J, Raschke P (2010): Zusammenhang zwischen Verschreibungsverhalten der Ärzte und Medikamentenabhängigkeit ihrer Patienten.

Bundesgesundheitsblatt Gesundheitsforschung Gesundheitsschutz 53(4), 319-325

IMS (Institut für Medizinische Statistik): Der pharmazeutische Markt in Deutschland (DPM); Frankfurt a. M. 2009

Kemper N, Poser W, Poser S (1980): Benzodiazepin-Abhängigkeit. Deut Med Wochenschr 105(49), 1707-1712

Kessler RC, Crum RM, Werner LA, Nelson CB, Schulenberg J, Anthony JC (1997): Lifetime co-occurence of DSM III alcohol abuse and dependence with other psychiatric disorders in the National Comorbidity Survey. Arch Gen Psychiatry 54(4), 313-321

Kremer R (2002) Clock drawing test in dementia: a critical review. Rev Neurol Arg 27, 223-227

Kvetina J, Guaitani PA (1969): Effect of diazepam on liver function of rats. Pharmacology 2(1), 17-20

Lader M (1991): History of benzodiazepine dependence. J Subst Abuse Treat 8(1-2), 53-59

Lader M (2011): Benzodiazepines revisited-will we ever learn? Addiction 106(12), 2086-2109

Lader MH, Ron M, Petursson H (1984): Computerized axial brain tomography in long-term benzodiazepine users. Psychol Med 14(1), 203-206 
Launchbury AP, Drake J, Seager H (1992): Misuse of temazepam. BMJ 305(6847), 252-253

Laux G, König W (1985): Benzodiazepine: Langzeiteinnahme oder Abusus? Ergebnisse einer epidemiologischen Studie. Dtsch Med Wochenschr 110(34), 1285-1290

Liu X, Ye W, Watson P, Tepper P (2010): Use of benzodiazepines, hypnotics, and anxiolytics in major depressive disorder. Association with chronic pain diseases. J Nerv Ment Dis 198(8), 544-550

Longo LP, Johnson B (2000) Addiction: Part 1 Benzodiazepines - Side effects, abuse risk and alternatives. Am Fam Physician 61(7), 2121-2128

Low K, Crestani F, Keist R, Benke D, Brunig I, Benson JA, Fritschy JM, Rulicke T, Bluethmann H, Möhler H, Rudolph U (2000): Molecular and neuronal substrate for the selective attenuation of anxiety. Science 290(5489), 131-134

Lucki I, Rickels K Geller AM (1986): Chronic use of benzodiazepines and psychomotor and cognitive test performance. Psychopharmacology (Berl) 88(4), 426-433

Lydiard RB (2003): The role of GABA in anxiety disorders. J Clin Psychiatry 64(suppl 3), $21-27$

Mader P: Basisinformationen Alkohol in: DHS Info; hrsg. v. der Deutschen Hauptstelle gegen die Suchtgefahren e.V., Hamm 2001

Manthey L, van Veen T, Giltay EJ, Stoop JE, Neven AK, Pennix BW, Zitman FG (2011): Correlates of (inappropriate) benzodiazepine use: the Netherlands Study of Depression and Anxiety (NESDA). Br J Clin Pharmacol 71(2), 263-272

Margraf J: Mini-Dips Diagnostisches Kurzinterview bei psychischen Störungen (Testmappe, Handbuch und Interviewerleitfaden); Springer, Berlin 1994

Martinez-Cano H, De Iceta Ibanez de Gauna M, Vela-Bueno A, Wittchen HU (1999): DSM III co-morbidity in benzodiazepine dependence. Addiction 94(1), 97-107

McAndrews MP, Weiss RT, Sandor P, Taylor A, Carlen PL, Shapiro CM (2003): Cognitive effects of long- term Benzodiazepine use in older adults. Hum Psychopharmacology 18(1), 51-57

Merikangas KR, Herrel R, Swendsen J, Rössler W, Aidacic-Gross V, Angst J (2008): Specificity of bipolar spectrum conditions in the comorbidity of mood and substance use disorders. Arch Gen Psychiatry 65(1), 47-52

Mol A, Gorgels W, Oude Voshaar RC, Breteler M, Van Balkom A, Van de Lisdonk E, Kan C, Zitman F (2005): Associations of benzodiazepine craving with other clinical variables in a population of general practice patients. Compr Psychiatry 46(5), 353-360

Moodley P, Golombok S, Shine P, Lader M (1993): Computed axial brain tomograms in long-term benzodiazepine users. Psychiatry Res $\underline{48(2), 135-144}$ 
O’Brien CP (2005): Benzodiazepine use, abuse, and dependence. J Clin Psychiatry 66(suppl 2), 28-33

Olsen RW, Sieghardt W (2008): International Union of Pharmacology. LXX. Subtypes of gamma-Aminobutyric Acid(A) Receptors: Classification on the basis of Subunit Composition, Pharmacology, and Function. Update. Pharamcol Rev $\underline{60}$, 243-260

Paterniti SP, Dufouil C, Alperovitch A (2001): Long- Term Benzodiazepine Use and Cognitive Decline in the Elderly: The Epidemiology of Vascular Aging Study. J Clin Psychopharmacol 22(3), 285-293

Pelissolo NMJ, Haddad RS, Baddoura C (2000): A general poulation survey on patterns of benzodiazepine use and dependence in Lebanon. Acta Psychiatr Scand (2000) 102(6), 429431

Perera KMH, Powell T, Jenner FA (1987): Computerized axial tomographic studies following long-term use of benzodiazepines. Psychol Med 17, 775-777

Poser W, Poser S: Medikamente - Mißbrauch und Abhängigkeit: Entstehung - Verlauf Behandlung; Georg Thieme, Stuttgart 1996

Poser W, Poser S, Roscher D, Argyrakis A (1983): Do benzodiazepines cause cerebral atrophy? Lancet $\underline{321}, 715$

Poser W, Böning J, Holzbach R, Schmidt LG: Medikamentenabhängigkeit (Sedativa, Hypnotika, Analgetika, Psychostimulanzien); in: Evidenzbasierte Suchtmedizin Behandlungsleitlinie Substanzbezogene Störungen; hrsg. v. Schmidt LG, Gastpar M, Falkai P, Gaebel W; Deutscher Ärzteverlag, Köln 2006, 271-300

Ray WA, Griffin MR, Schaffner W, Bauth DK, Melton LJ (1987): Psychotropic drug use and the risk of hip fracture. N Engl J Med 316, 363-369

Rickels K, Schweizer E, Case WG, Greenblatt DJ (1990): Long term therapeutic use of benzodiazepines: Effects of abrupt discontinuation. Arch Gen Psychiatry 47(10), 899-907

Rickels K, Garcia-Espana F, Mandos L, Case GW (2008): Physician Withdrawal Checklist (PWC-20). J Clin Psychopharmacol 28(4), 447-451

Rosenbaum JF (2005): Attitudes towards benzodiazepines over the years. J Clin Psychiatry $\underline{66}$ (suppl 2), 4-8

Ross HE (1993): Benzodiazepine use and anxiolytic abuse and dependence in treated alcoholics. Addiction $\underline{88}, 209$

Roy-Byrne P (2005): The GABA-benzodiazepine receptor complex: structure, function, and role in anxiety. J Clin Psychiatry 66(suppl 2), 14-20

Rudolph U, Crestani F, Benke D (1999): Benzodiazepine actions mediated by specific gamma-aminobutyric acid A9 receptor subtypes. Nature 401(6755), 796- 800 
Saari TI, Uusi-Oukari M, Ahonen J, Olkkola KT (2011): Enhancement of GABAergic activity: neuropharmacological effects of benzodiazepines and therapeutic use in anesthesiology. Pharmacol Rev $\underline{63}$, 243-267

Schmauss C, Krieg J-C (1987): Enlargement of cerebrospinal fluid spaces in long-term benzodiazepine abusers. Psychol Med 17(4), 869-873

Schmidt LG, Konrad N, Rommelspacher H, Schmidt K, Singer M, Teyssen S:

Alkoholabhängigkeit (Suchtmedizinische Reihe, Band 1); hrsg. v. Wissenschaftlichen Kuratorium der deutschen Hauptstelle für Suchtfragen (DHS), Hamm 2003

Schneider U, Altmann A, Baumann M, Bernzen J, Bertz B, Bimber U, Broese T, Broocks A, Burtscheidt W, Cimander KF (2001): Comorbid anxiety and affective disorder in alcoholdependent patients seeking treatment: the first Multicentre Study in Germany. Alcohol Alcoholism 36(3), 219-223

Schweizer E, Rickels K, Case WG (1990): Long-term therapeutic use of benzodiazepines, II: effects of gradual taper. Arch Gen Psychiatry 47, 908-915

Schweizer E, Rickels K, Uhlenhuth EH: Issues in the long-term treatment of anxiety disorders; in: Psychopharmacology: the fourth generation of progress; hrsg. v. Bloom FE, Kupfer DJ; Raven Press, New York 1995, 1349-1359

Shulman KI (2000): Clock-drawing: is it the ideal cognitive screening test? Int J Geriatr Psychiatry 15(6), 548-561

Shulman KI, Gold D, Cohen C, Zucchero C (1993) Clock drawing and dementia in the community: a longitudinal study. Int J Geriatr Psychiatry $\underline{8}$, 487- 496

Smoldersa M, Lauranta M, van Rijswijkb E, Muldera J, Braspenninga J, Verhaakc P, Wensinga M, Grola R (2007): The impact of co-morbidity on GPs' pharmacological treatment decisions for patients with an anxiety disorder. Fam Pract 24, 538-546

Stewart SA (2005): The Effects of Benzodiazepines on Cognition. J Clin Psychiatry 66(suppl 2), 9-13

Tan KR, Brown M, Labouebe G, Yvon C, Creton C, Fritschy J, Rudolph U, Lüscher C (2010): Neural bases for addictive properties of benzodiazepines. Nature 463(7282), 769-774

Tata PR, Rollings J, Collins M, Pickering A, Jacobsen RR (1994): Lack of cognitive recovery following withdrawal from long-term benzodiazepine use. Psychol Med $\underline{9}$, 313-318

Verdoux H, Lagnaoui R, Begaud B (2004): Is benzodiazepine use a risk factor for cognitive decline and dementia? A literature review of epidemiological studies. Psychol Med 35(3), 307-315

Voshaar RC, Couvee JE, Van Balkom AJ, Mulder PG, Zitman FG (2006): Strategies for discontinuing long-term benzodiazepine use: meta analysis. Br J Psychiatry $\underline{189}$, 213-220 
WHO (World Health Organization): Tenth revision of the international classification of diseases; WHO Genf 1991

Wittchen HU: Natural course and spontaneous remissions of untreated anxiety disorders: results of the Munich Follow-up Study (MFS); in: Panik and Phobies 2: treatment and variables affecting course and outcome; hrsg. v. Hand I und Wittchen HU; Springer, Berlin 1988, 3-17

Wolf ME (2003): LTP may trigger addiction. Mol Interv 3(5), 248-252 


\section{Danksagung}

Ich danke Frau Prof. Dr. Ursula Havemann-Reinecke für die Überlassung des Themas, für ihre intensive Betreuung und ihre anregende Kritik.

Mein besonderer Dank gilt Herrn PD Dr. Dirk Wedekind. Seine Fähigkeit, andere in besonderer Weise zu motivieren, seine konstruktiven Anregungen sowie die schnellen Korrekturen haben mir bei der Durchführung und Fertigstellung der Arbeit sehr geholfen.

Des Weiteren möchte ich mich bei Frau Dr. med. Scherf-Geschke bedanken, die mir insbesondere zu Beginn der klinischen Studie mit viel Rat und Hilfe zur Seite stand.

Nicht zuletzt möchte ich mich bei allen Mitarbeitern der Psychiatrischen Abteilung der Universitätsklinik Göttingen für ihre Unterstützung bedanken. Außerdem danke ich den Patienten, die durch ihre aktive Mitarbeit die Durchführung der vorliegenden Studie überhaupt erst ermöglicht haben. 


\section{Lebenslauf}

Ich wurde am 20.12.1979 in Bremen geboren und machte dort am Gymnasium an der Hamburger Straße 1999 das Abitur. Ich begann anschließend Soziologie an der Uni Bremen zu studieren, trat aber 2000 ein „Freiwilliges Soziales Jahr“ im Rote Kreuz Krankenhaus in Bremen an.

Im WS 2001/2002 nahm ich das Studium der Humanmedizin an der Georg-AugustUniversität in Göttingen auf, absolvierte im März 2003 das Physikum und im März 2004 das erste Staatsexamen. In 2005 famulierte ich u.a. in der Abteilung für Neurologie des Kantonsspitals Aarau, Schweiz. Mein Praktisches Jahr begann ich August 2006 im Weender Krankenhaus in Göttingen, verbunden mit einem weiteren Auslandsaufenthalt an der University of the West Indies (Gynäkologie), Barbados. Im Mai 2008 schloß ich mein Studium mit der Ärztlichen Prüfung ab.

Im August 2008 begann ich meine Facharztausbildung für zwei Jahre in der Abteilung für Innere Medizin des Weender Krankenhauses in Göttingen (Prof. Dr. Karaus), die ich von März 2011 bis März 2012 in der Abteilung für Allgemeinchirurgie und Gefäßchirurgie des Krankenhauses Neu-Bethlehem in Göttingen (Dr. Loweg) fortsetzte. Von März bis Oktober 2012 arbeitete ich in der in der Abteilung für Geriatrie des Weender Krankenhauses (Prof. Dr. Nau). Im Februar 2013 wechselte ich in die Abteilung für Innere Medizin (Dr. Pott) im Krankenhaus Buchholz. 